\title{
Kinematic Control and Posture Optimization of a Redundantly Actuated Quadruped Robot
}

\author{
Travis Thomson \\ McGill University \\ Montreal, Quebec \\ December 2011 \\ Master of Engineering \\ Department of Mechanical Engineering
}

A thesis submitted to McGill University in partial fulfillment of the requirements of the degree of Master of Engineering

(C) Travis Thomson, 2011 


\section{ACKNOWLEDGEMENTS}

First and foremost, I would like to thank my supervisor Dr. Inna Sharf for her unfaltering guidance and help during the course of my degree. Her patience, supervision and financial support throughout my research have made this thesis possible. I wish to thank my lab mate Korhan Türker for the helpful exchange of ideas over the past two years. I would like to thank Blake Beckman, our contact at DRDC-Suffield, for supplying me a laptop with all the necessary software and information installed. I would also like to thank my girlfriend Erica Dancose for the near infinite number of times she has supported me and kept me fed during my long hours in front of a computer screen. I also wish to thank my family for the continuous support and my fellow lab mates, Hashim Mazhar, Chris Prahacs and Thai Chau for their helpful nature. 


\begin{abstract}
Although legged locomotion for robots has been studied for many years, the research of wheeled-leg robotics is much more recent. Robots of this type can take advantage of the energy efficiency of wheeled locomotion while adapting to more difficult terrain with legged locomotion when necessary. The Micro Hydraulic Toolkit (MHT), developed by engineers at Defence Research and Development Canada (DRDC), is a good example of such a robot. MHT is an unmanned quadruped hybrid robot with hydraulically articulated legs and electric wheels. Investigation into the control of MHT leads to a better understanding of ground vehicle control for terrestrial exploration and reconnaissance. The methodology applied in this work uses MHT's velocity kinematics to determine joint rates for the given posture and trajectory inputs. This thesis will review the formulation of the kinematic controller and the results obtained via co-simulation using Matlab’s Simulink and the high-fidelity model of MHT in LMS Virtual Lab.
\end{abstract}




\section{SOMMAIRE}

Il y a déjà plusieurs années que les robots à locomotion articulée font sujets de maintes études, la recherche visant la locomotion articulée sur roues, par contre, est beaucoup plus récente. Les robots de ce type peuvent bénéficier de l'efficacité énergétique de la locomotion articulée sur roues tout en s'adaptant à des environnements plus difficiles par l'utilisation de la locomotion articulée lorsque nécessaire. Le toolkit micro-hydraulique (Micro Hydraulic Toolkit (MHT)), conçu par des ingénieurs au Recherche et développement pour la défense Canada (RDDC), est un bon exemple d'un tel robot. MHT est un robot quadrupède hybride autonome composé de pattes articulées hydrauliques et de roues électriques. La recherche ciblant les mécanismes de control du MHT mène à une meilleure compréhension du control nécessaire pour l'exploration et la reconnaissance que font les véhicules terrestres. La méthodologie employer pour ce travail utilise la vélocité cinématique du toolkit micro-hydraulique pour déterminer la proportion des articulations pour les positions voulues et les trajectoires émises. Cette thèse revisera la formulation des commandes cinématiques et des résultats obtenus par l'entremise de co-simulations utilisant Matlab's Simulink et le modèle haute-fidélité de MHT dans LMS Virtual Lab. 


\section{TABLE OF CONTENTS}

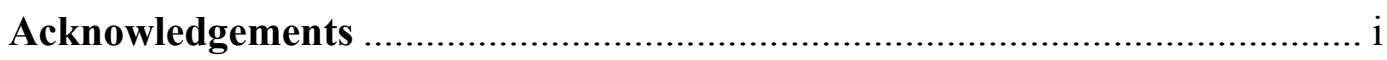

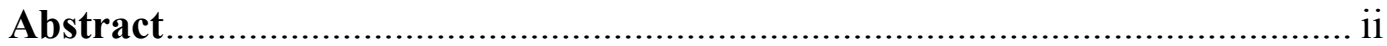

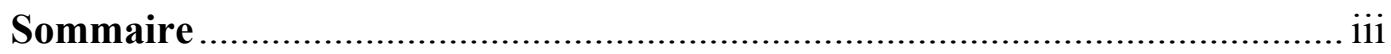

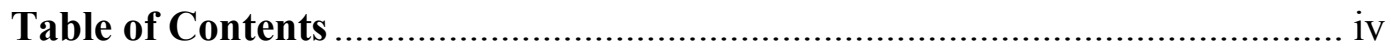

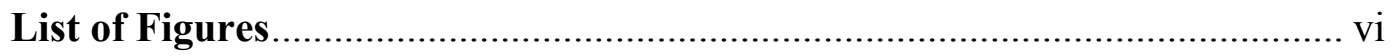

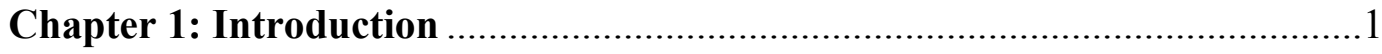

1.1. Wheeled/Tracked Locomotion............................................................2

1.2. Legged Locomotion .............................................................................. 3

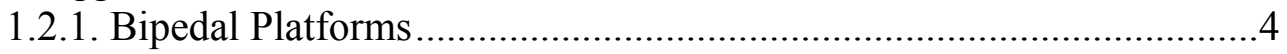

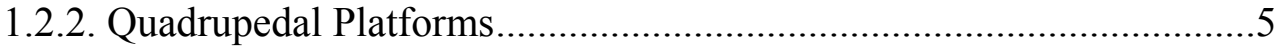

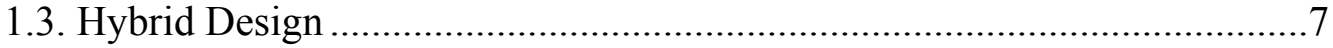

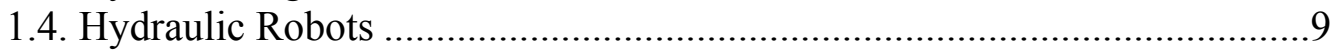

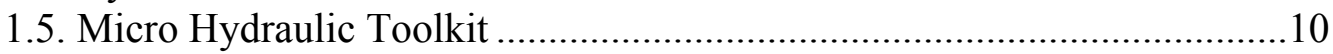

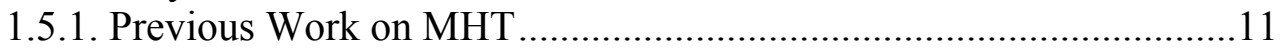

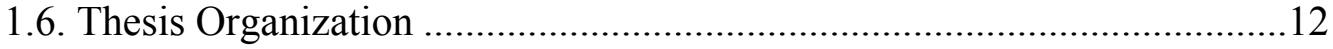

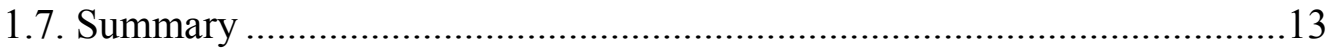

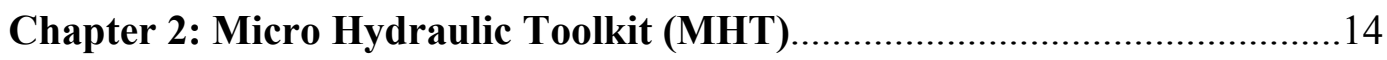

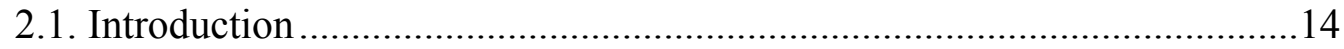

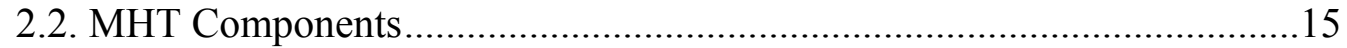

2.3. SimMechanics and MSC.ADAMS Models ............................................15

2.4. LMS Virtual Lab Model.........................................................................19

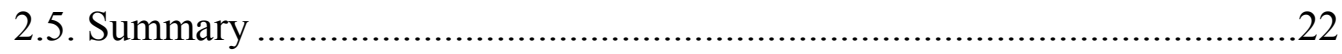

Chapter 3: Inverse Kinematics Controller .................................................23

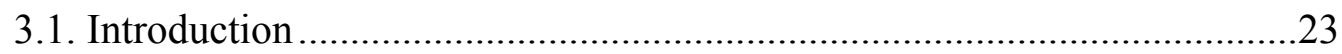

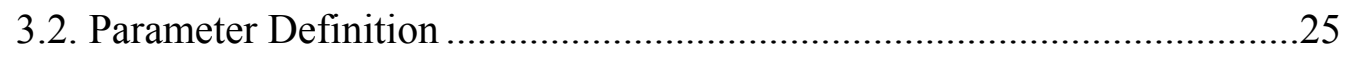

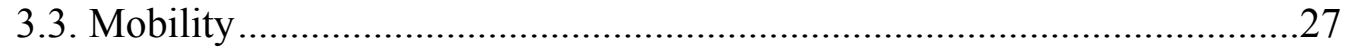

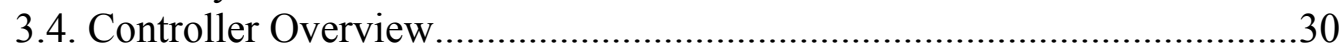

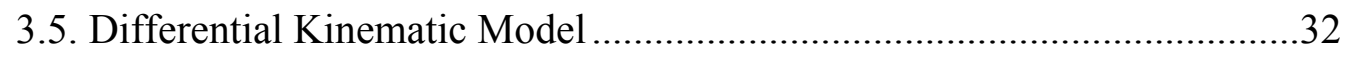

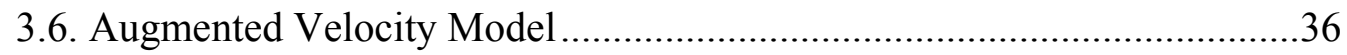

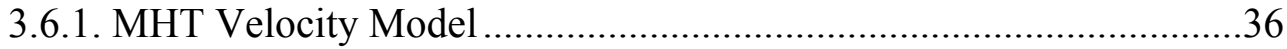

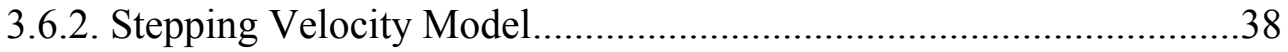

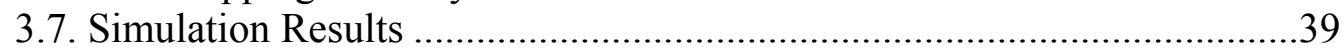

3.7.1. Vertical Motion Test Case ..............................................................40

3.7.1.1. MSC.ADAMS Simulation Results .......................................42 
3.7.1.2. LMS Simulation Results.......................................................43

3.7.2. Uneven Terrain Test Case ............................................................45

3.7.3. Independent Leg Control Test Case ............................................47

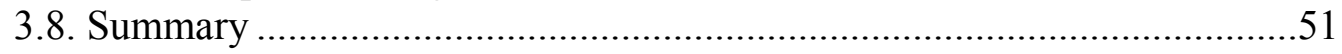

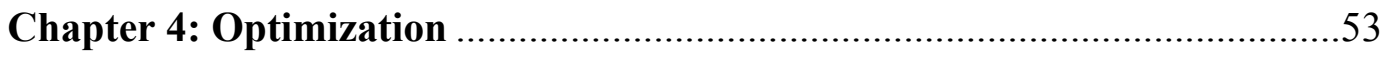

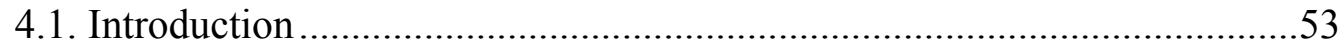

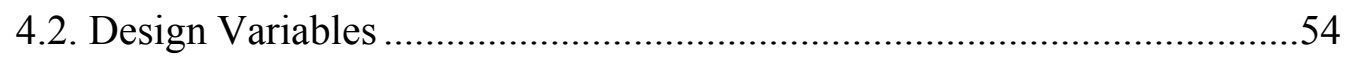

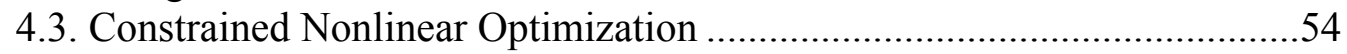

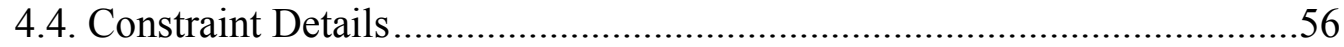

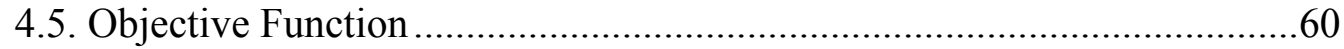

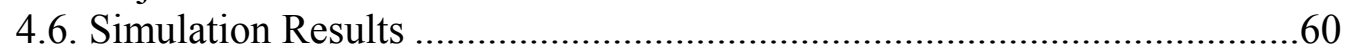

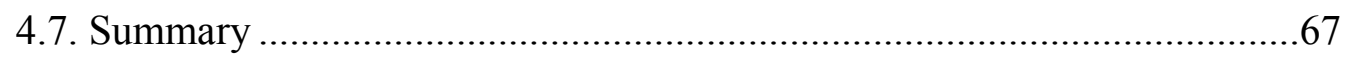

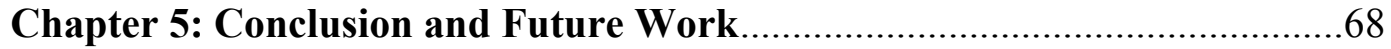

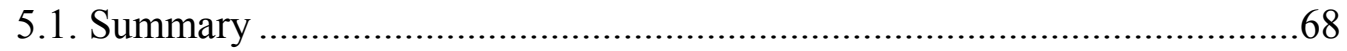

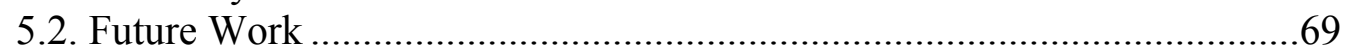

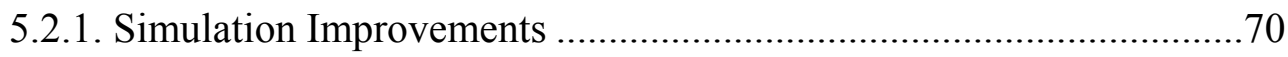

5.2.2. Additional features on physical robot............................................70

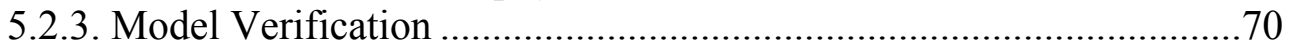

5.2.4. Controller Improvements....................................................... 71

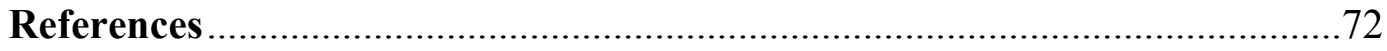

Appendix A 


\section{LIST OF FIGURES}

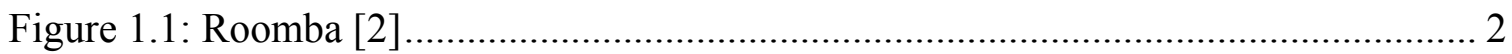

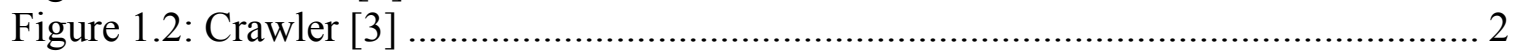

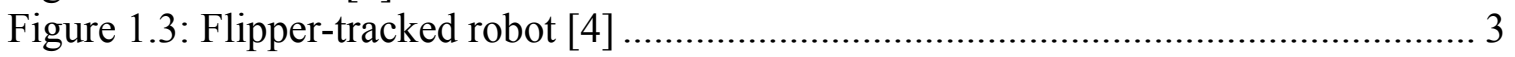

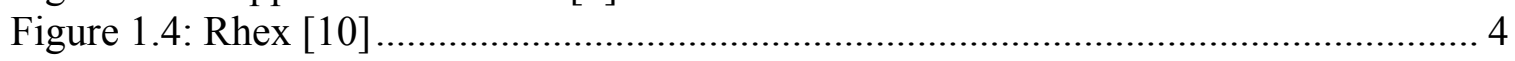

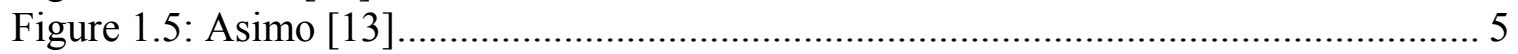

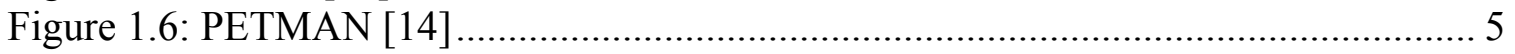

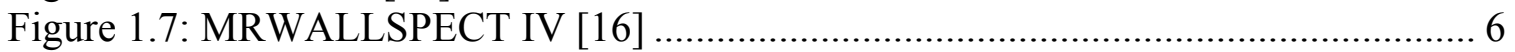

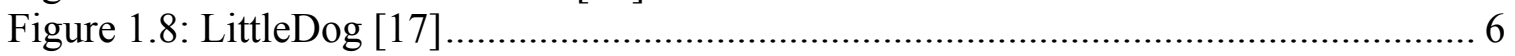

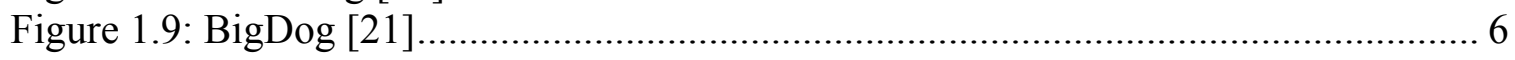

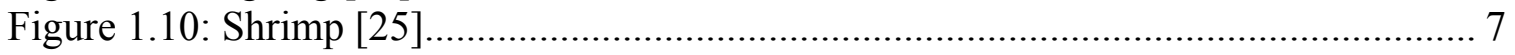

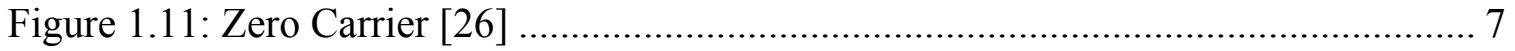

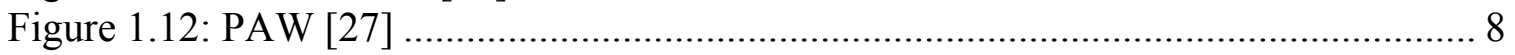

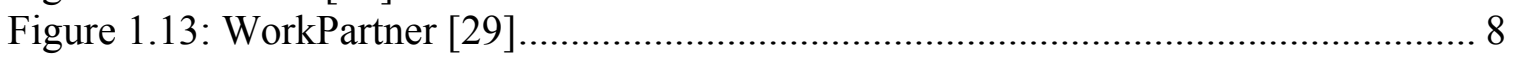

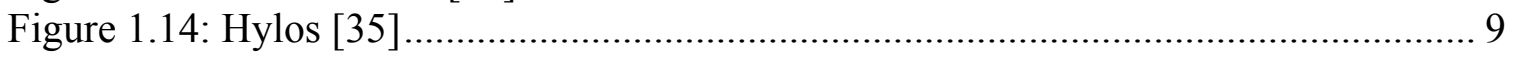

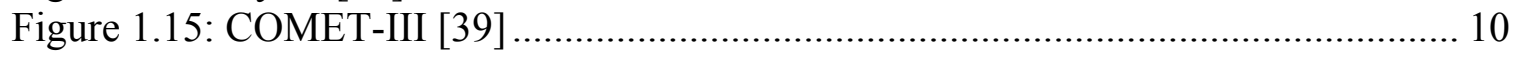

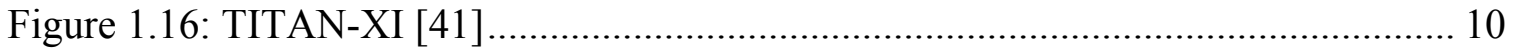

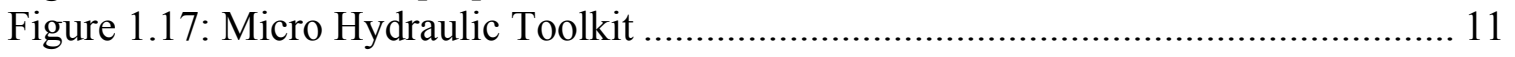

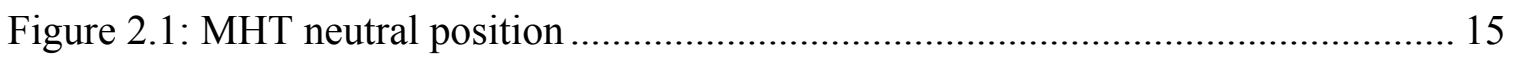

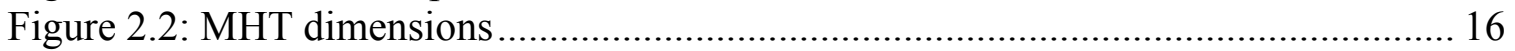

Figure 2.3: Micro Hydraulic Toolkit main components .............................................. 17

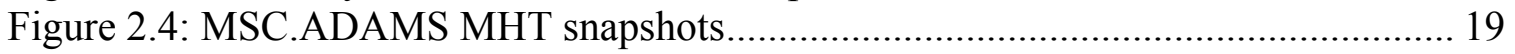

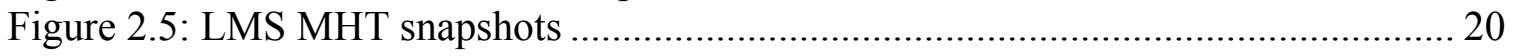

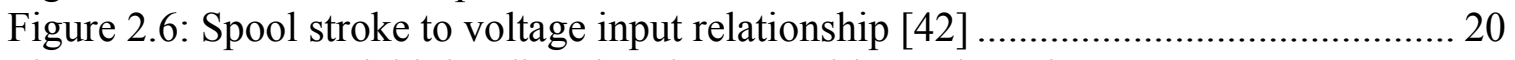

Figure 2.7: LMS model joint direction due to positive voltage input............................ 21

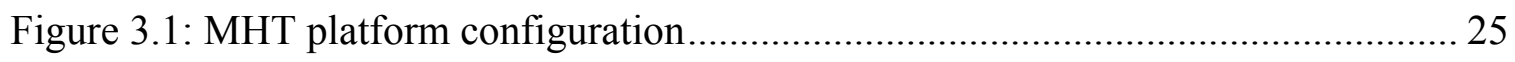

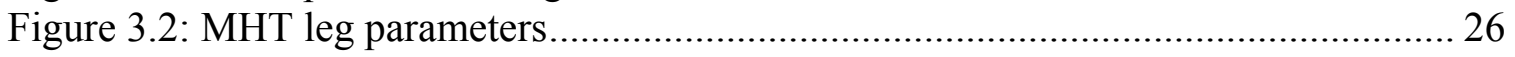

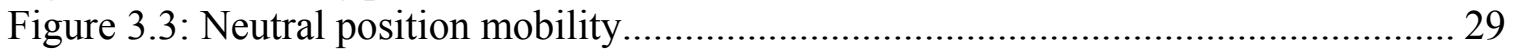

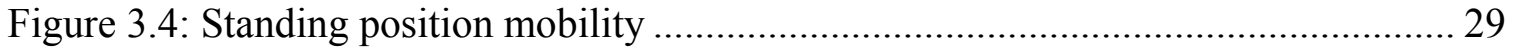

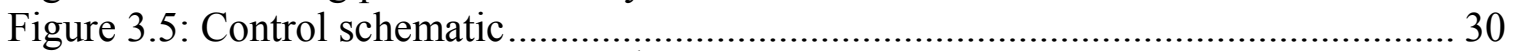

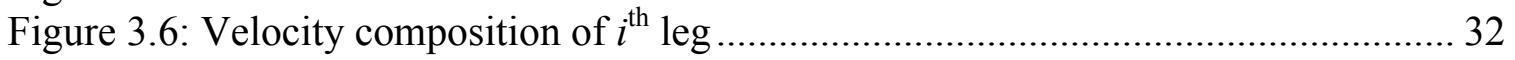

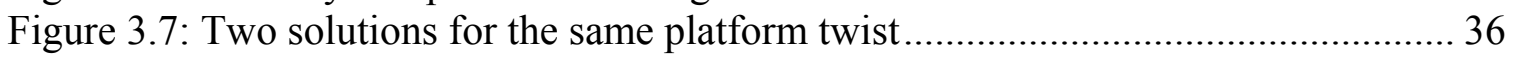

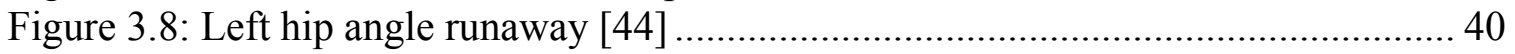

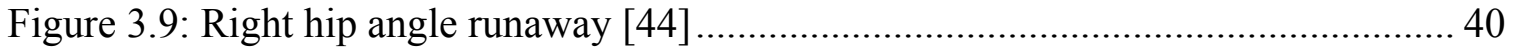

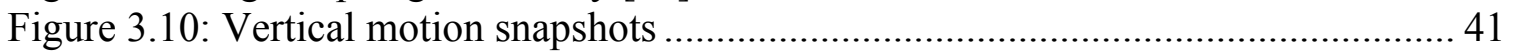

Figure 3.11: MSC.ADAMS vertical motion roll tracking ......................................... 42

Figure 3.12: MSC.ADAMS vertical motion pitch tracking......................................... 42

Figure 3.13: MSC.ADAMS vertical motion height tracking...................................... 42

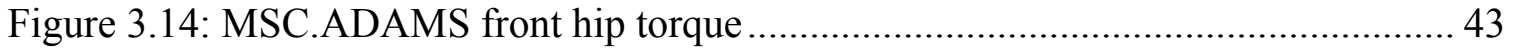

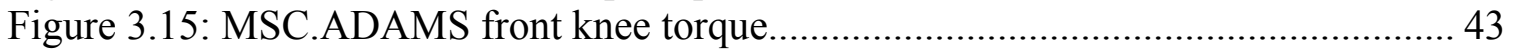

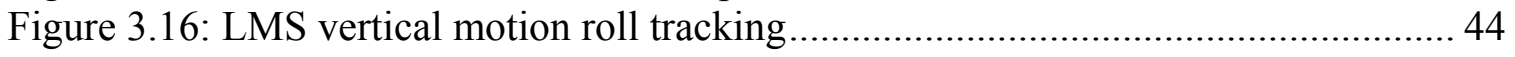

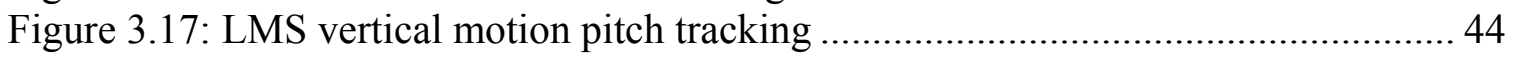

Figure 3.18: LMS vertical motion height tracking .................................................. 44 
Figure 3.19: LMS vertical motion wheel positions ................................................. 44

Figure 3.20: Uneven terrain height profiles ........................................................... 45

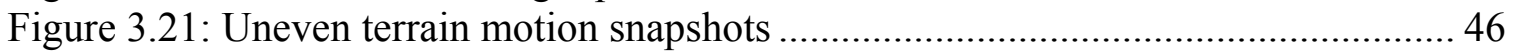

Figure 3.22: LMS uneven terrain motion roll tracking............................................... 46

Figure 3.23: LMS uneven terrain motion pitch tracking ............................................. 46

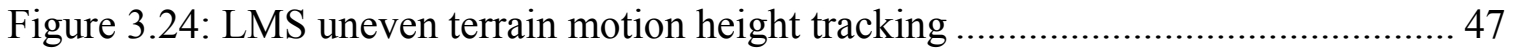

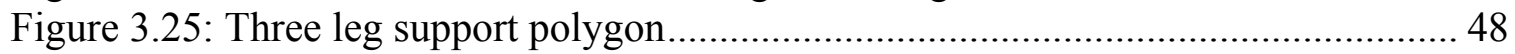

Figure 3.26: LMS stepping motion trajectory input ................................................ 48

Figure 3.27: Leg lift manoeuvre snapshots.............................................................. 49

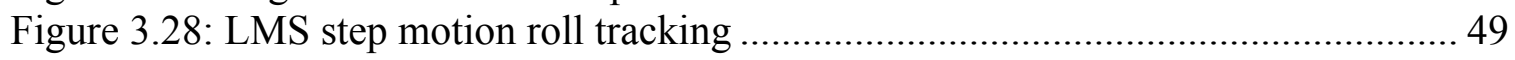

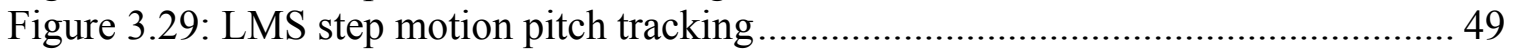

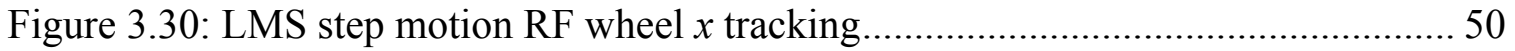

Figure 3.31: LMS step motion LF wheel $x$ tracking .............................................. 50

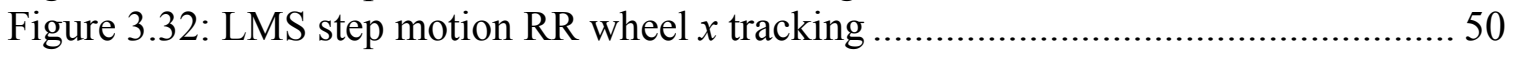

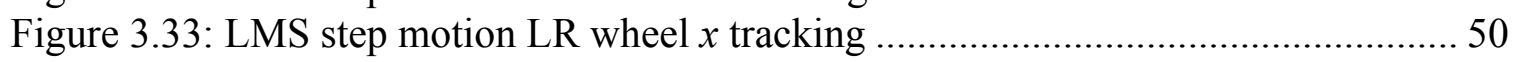

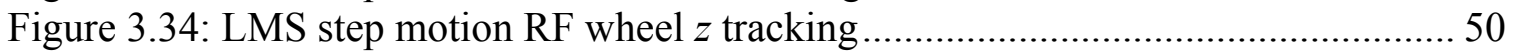

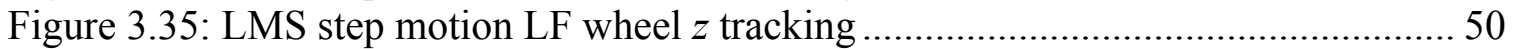

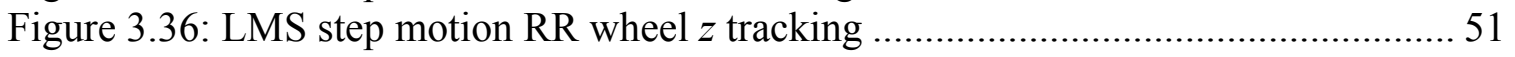

Figure 3.37: LMS step motion LR wheel $z$ tracking.............................................. 51

Figure 4.1: Minimum allowable height for optimization.............................................. 57

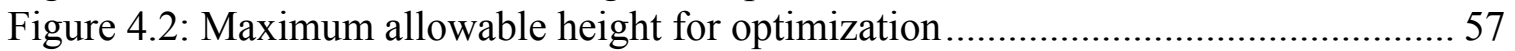

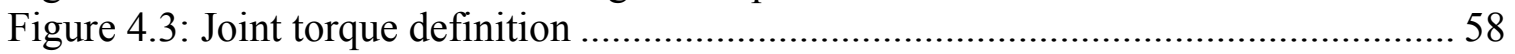

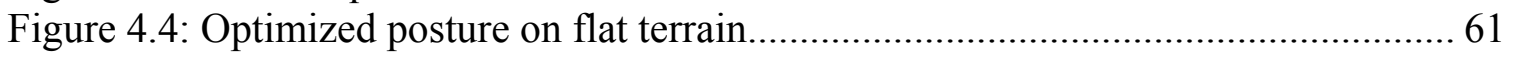

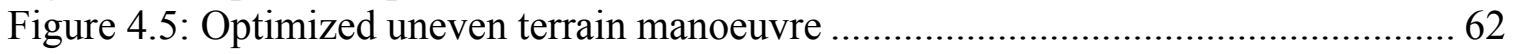

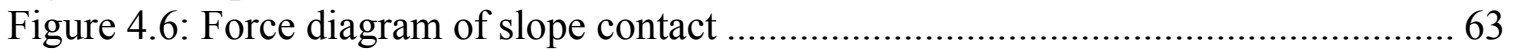

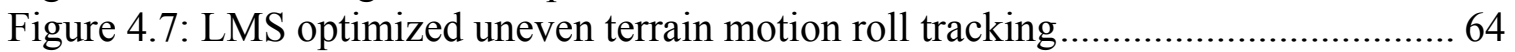

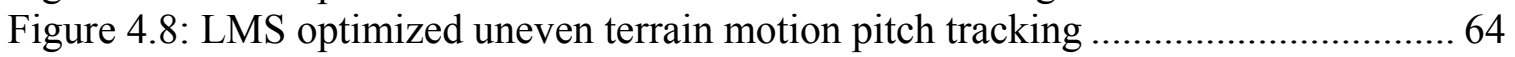

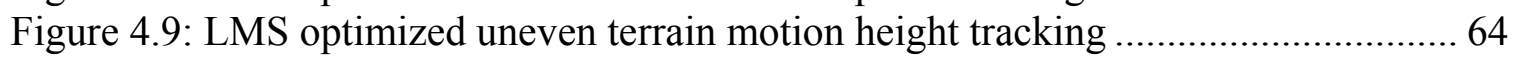

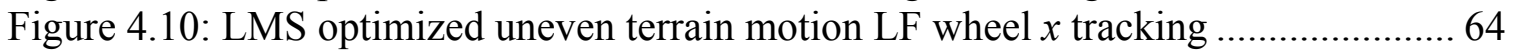

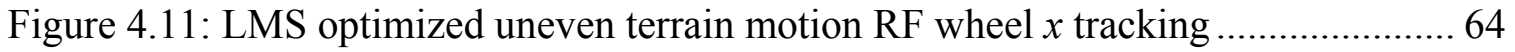

Figure 4.12: LMS optimized uneven terrain motion LR wheel $x$ tracking ..................... 65

Figure 4.13: LMS optimized uneven terrain motion RR wheel $x$ tracking...................... 65

Figure 4.14: LMS uneven terrain motion LF leg actuator torques comparison................ 66

Figure 4.15: LMS uneven terrain motion RF leg actuator torques comparison ............... 66

Figure 4.16: LMS uneven terrain motion LR leg actuator torques comparison ...............66

Figure 4.17: LMS uneven terrain motion RR leg actuator torques comparison ............... 66

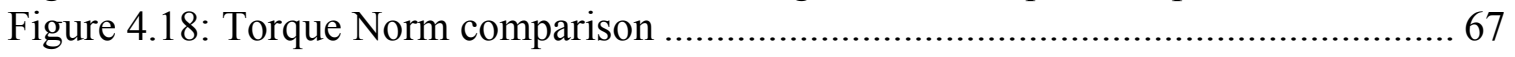




\section{Chapter 1: Introduction}

Powered mechanical locomotion has been in existence for over a century, creating a vast number of possibilities in numerous fields of research. The development of automotives has redefined the society around us, whether it is transportation, the logistics of corporations or even exploring a different planet remotely. Mechanically powered vehicles have created an extension to human movement, to facilitate and improve the way we live. Though this concept has been thoroughly tested in terms of speed and distance, it lacks the finesse that a human or animal body can offer in terms of versatility and flexibility. Most automobiles are built primarily for use on level terrain, such as relatively flat roads, to operate at maximum efficiency; however, this is very limiting in terms of mobility. Off-road vehicles have been developed as an appropriate response to extending a vehicle's mobility, all this by increasing the power input of an engine and/or supplying a better suspension system. However, the vehicles themselves remain particularly rigid and still succumb to the conductor's commands. Ideally, a vehicle being used for a given task would complete its mission in the most efficient manner possible and, maybe even more importantly, autonomously.

As complex electronics began to manifest in the $20^{\text {th }}$ century, robotics have overlapped with the influence of powered locomotion. Mobile robotics has allowed an even further extension of the concept of automotive design, without the need to rely on an onboard 
operator or even conventional auto mechanics. This allows the design of more versatile mechanics to accommodate complex terrain and/or potentially hazardous areas, where human injury might occur. The research on the issue of complex terrain adaptation has led to many interesting uses of various mobile robotic designs and applications.

\subsection{Wheeled/Tracked Locomotion}

A number of commercial robots have been available over the past decade with diverse functionality, such as conducting household chores or even providing extra security measures. These robots take advantage of long-standing vehicle concepts, such as wheels or treads, applied on a smaller scale and combined with sensors to allow for sensory feedback. Figure 1.1 shows the popularized vacuum robot Roomba by iRobot [1, 2], which can clean level floors autonomously. While cleaning, Roomba can also navigate around obstacles in its path using its on board sensors. Moving from household chores, to security, Figure 1.2 shows a robot created by Superdroid, called Crawler [3], which can be remotely operated with an equipped vision system. This robot's main function is remote surveillance, as it has an infrared camera capable of $360^{\circ}$ rotation.

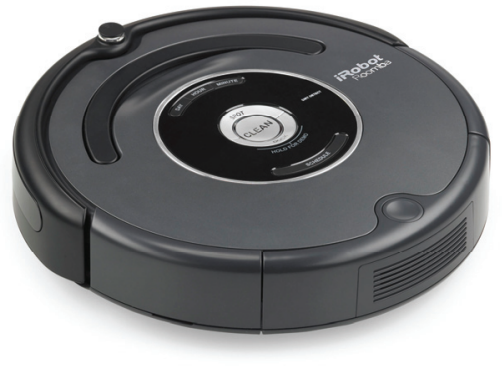

Figure 1.1: Roomba [2]

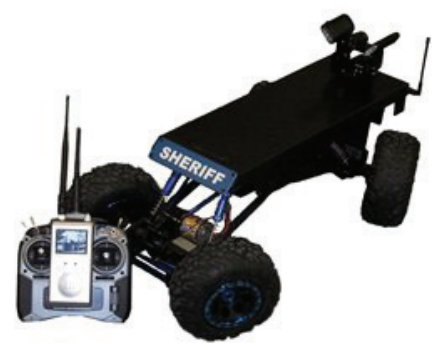

Figure 1.2: Crawler [3]

However effective in their own environment, these types of robots rely on classic automotive style concepts of terrain accommodation. Though efficient in energy use, this 
style of locomotion does not present much reliability on more complicated terrain such as stairs or other complex topography. As efficient as wheels are, tank-like tracks offer much more power for terrain traversal at the cost of energy use. The reconfigurable tracked robot presented by Mutka and Kovacic [4] (see Figure 1.3) demonstrates the added benefits of combining tracked locomotion and the ability to adjust to terrain types. The flipper-tracked robot's controller functions using the system's kinematics to develop posture control using twist theory [4]. This control technique has proven to be effective in simulation trials with regards to roll and pitch control.

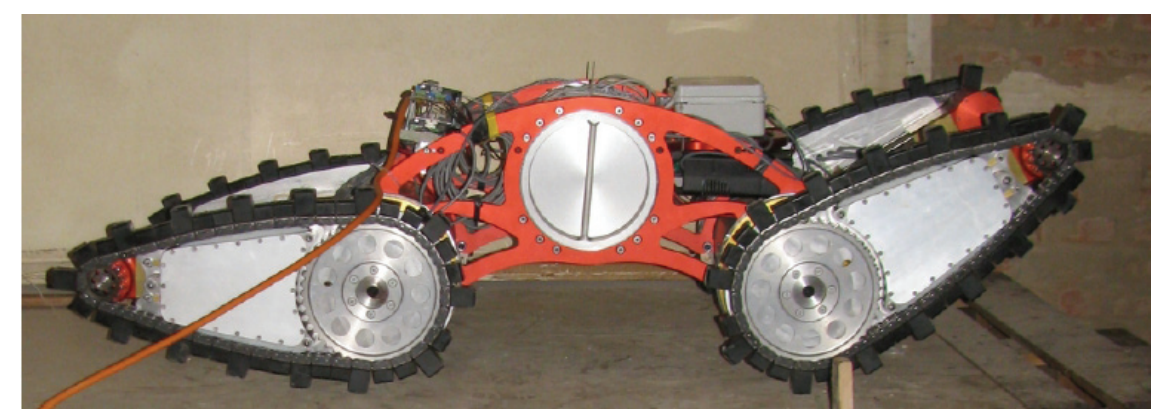

Figure 1.3: Flipper-tracked robot [4]

\subsection{Legged Locomotion}

A more recent locomotion that has been investigated in numerous robotic research projects is legged locomotion. This type of movement is often mimicry of a specific living organism's methods of motion, which allows for much more robustness when encountering non-level terrain topology. Many robots are based on the movement of animals or insects [5-7], such as RHex [8, 9], whose movement was developed based on the concept of a cockroach, as shown in Figure 1.4. 


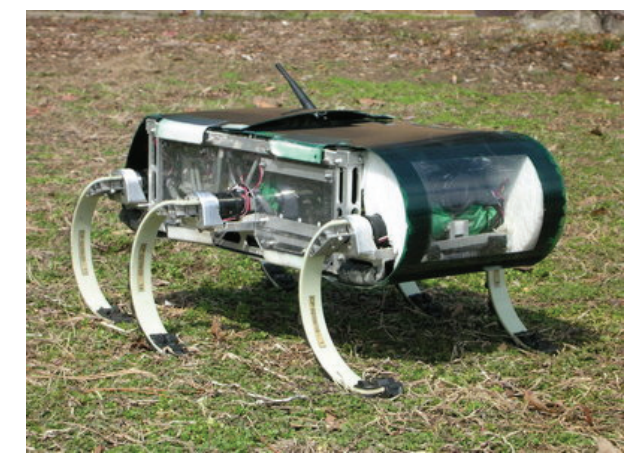

Figure 1.4: Rhex [10]

RHex serves as an effective all-terrain vehicle for its size with a reasonably simple design model. With six actuators, one for each leg, RHex offers a straightforward platform for controller development as well.

\subsubsection{Bipedal Platforms}

Bipedal forms of locomotion are the most familiar to us, however they introduce a number of stability problems that mechanics and software control must accommodate. The complexity of the human anatomy still far surpasses any mechanical replica, both in terms of stability and even energy efficiency. The dozens of muscles included in a human leg serve as tailored actuators fuelled by our diets, allowing for significant strength and flexibility. This concept is far too complicated to replicate in a mechanical format precisely, therefore a legged robot is often built using fewer, carefully placed actuators. An excellent example of a humanoid robot is Honda's Asimo [11, 12] shown in Figure 1.5. Asimo originates from a long line of bipedal-legged prototypes and is composed of numerous rotary joints. 


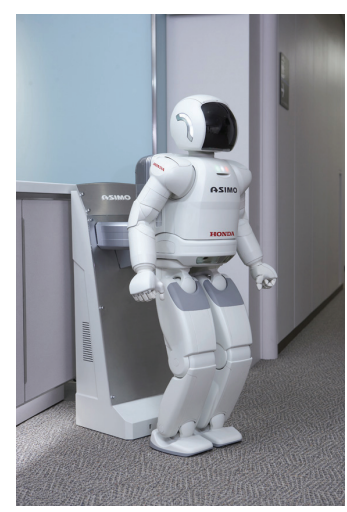

Figure 1.5: Asimo [13]

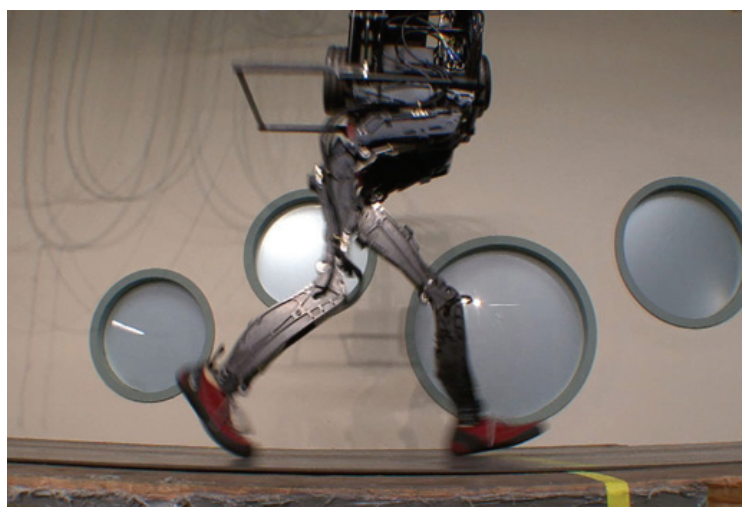

Figure 1.6: PETMAN [14]

Boston Dynamics has also demonstrated the effectiveness of bipedal motion with their robot PETMAN [14] (see Figure 1.6) which can even stabilize after encountering an unpredictable external force. PETMAN is an adaptation on the BigDog platform presented in the next subsection.

\subsubsection{Quadrupedal Platforms}

Quadruped leg design in robotics improves many of the stability issues that arise in bipedal structures. Rather than relying on only one leg for support during a stepping motion, up to three legs remain to stabilize a body more effectively during mid-step. Some robots use the added stability and contact points of quadrupedal design to create walking and running gaits similar to that of animals. The robot, MRWALLSPECT IV [15] (see Figure 1.7), shows the effectiveness, not only of legged motion, but in using range and gyroscope sensors to recreate its immediate environment in real-time [16]. With the information of the terrain ahead, it can implement a posture planning algorithm to adapt to the ground topology. 


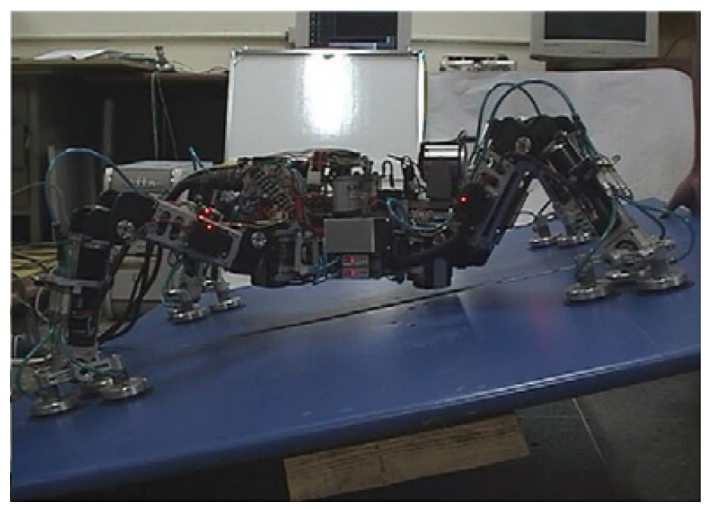

Figure 1.7: MRWALLSPECT IV [16]

A good example of quadruped design are the LittleDog [17-19] and BigDog [20, 21] robots developed at MIT, shown in Figures 1.8 and 1.9, respectively. Both of these robots use the same method of locomotion, however on different scales.

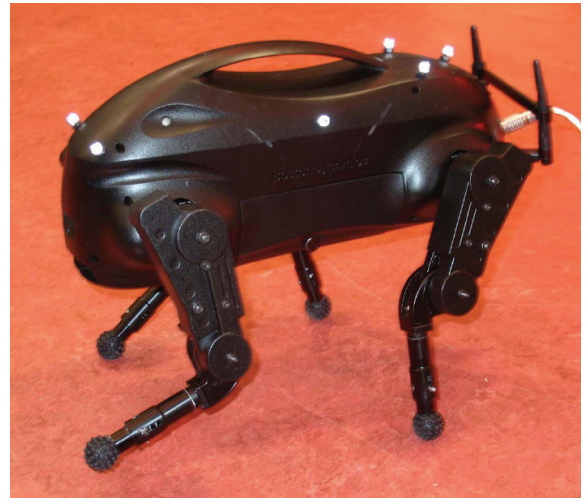

Figure 1.8: LittleDog [17]

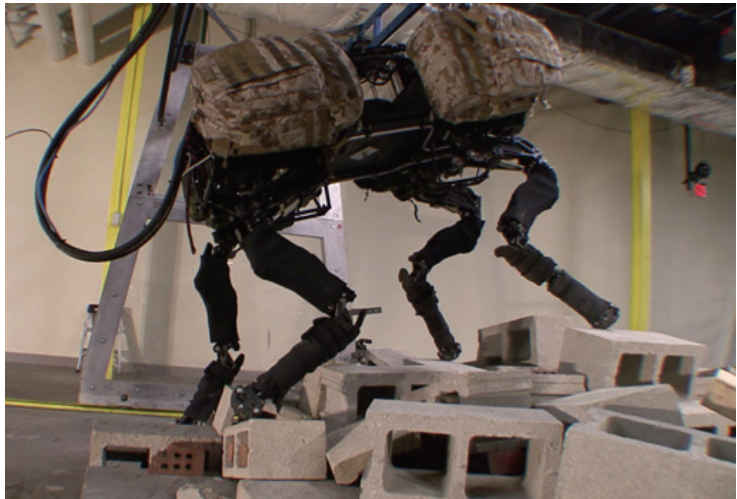

Figure 1.9: BigDog [21]

A robot like Big Dog offers excellent all-terrain capability, being able to clear many obstacles by easily walking over them. Though BigDog's locomotion style is effective and it has a considerable payload capacity, it requires a gasoline engine to operate, as it needs a significant amount of power. Therefore, it can be seen how the drawback of legged locomotion is the high energy requirements to generate the necessary thrust forces to move the body further and faster [22]. 


\subsection{Hybrid Locomotion}

Hybrid locomotion involves combining the strengths of legged and wheeled mobile platforms. This form of motion provides robots with a significant benefit at the cost of a more complex control system. Walking mechanisms alone tend to have high-energy requirements, as there are numerous constantly moving joints, though they have the advantage of more adaptability with complex terrain. On the other hand, wheeled motion provides more efficient energy use, however is limited to simpler terrain, reducing its mobile options. With hybrid robots, the advantages of both wheeled and legged locomotion can be employed to negate each of the type's respective weaknesses. A good example of this is the Shrimp robot [23], which uses a passive joint at its center to adjust to terrain geometry. This passive joint provides support and adaptability while climbing step terrain features, as shown in Figure 1.10. Another example of a stair climbing robot is the Zero-Carrier [24], built to provide wheel chairs with the ability to climb stair cases. The design of this robot focuses on many support points with prismatic joints and range sensors to locate and climb step features, as shown in Figure 1.11.

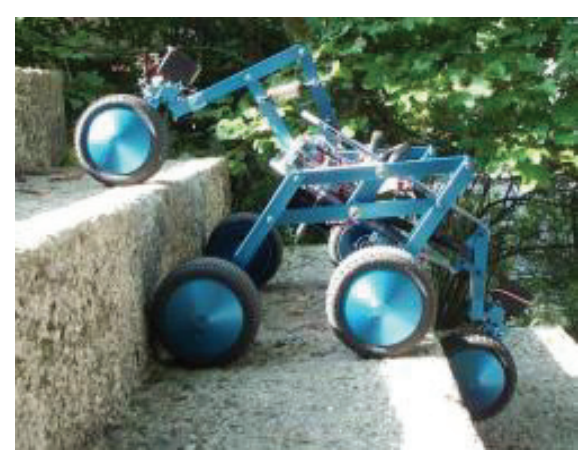

Figure 1.10: Shrimp [25]

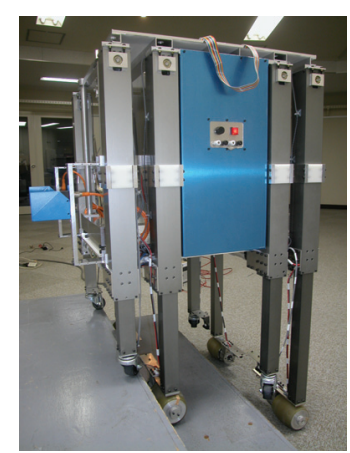

Figure 1.11: Zero Carrier [26]

A robot making efficient use of a minimum number of joints and hybrid capabilities is the Platform for Ambulating Wheels (PAW) robot [27, 28]. This versatile robot shown in 
Figure 1.12 in a bounding gait, though underactuated, is able to move in several different gaits with the use of its hybrid style setup and even perform jumping manoeuvres.

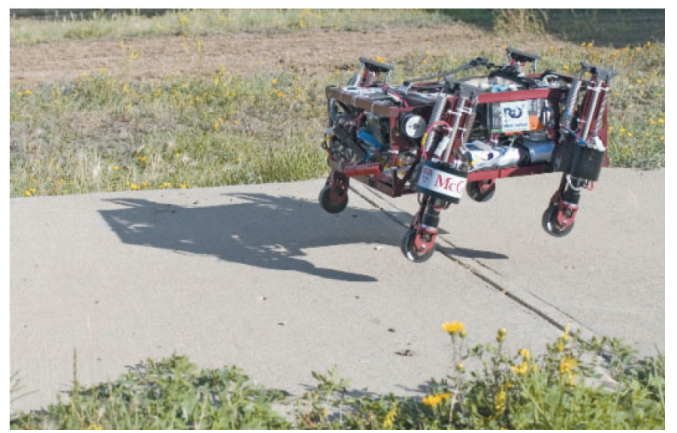

Figure 1.12: PAW [27]

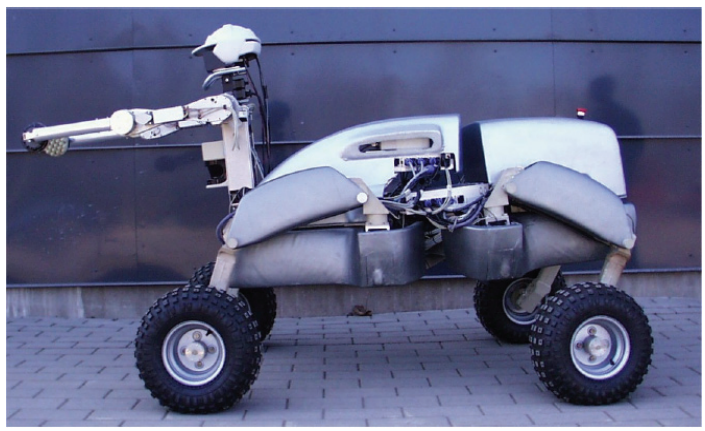

Figure 1.13: WorkPartner [29]

WorkPartner [29-31] (see Figure 1.13), a hybrid robot developed at the Helsinki University of Technology also shows the benefits of this style of movement. In addition to the use of hybrid locomotion, WorkPartner has a vision system and two serial manipulator arms for interacting with its direct environment as well, proving to be a versatile machine.

Finally, the hybrid quadruped robot Hylos [32-37], developed at the Pierre and Marie Curie University, serves as an inspiration for the controller presented in this thesis. Hylos is a relatively lightweight and small robot with pivoting wheels shown to efficiently adapt to uneven terrain (see Figure 1.14). Unlike PAW, Hylos has a slightly more complex leg structure, with an actuated knee joint that provides added manoeuverability. Hylos serves as an excellent demonstration of the advantages of hybrid quadruped motion, using the combination of energy efficiency of wheels and terrain adaptability of its legs. 


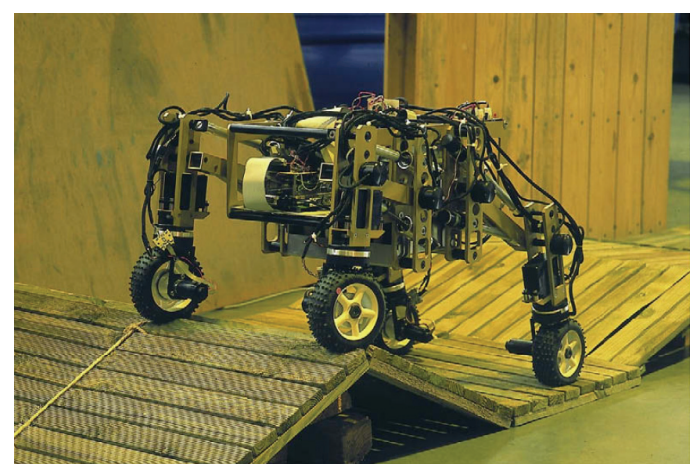

Figure 1.14: Hylos [35]

\subsection{Hydraulic Robots}

Aside from the modes of locomotion a robot might use, it is also critical to consider the actuators controlling that locomotion. Very often, electric actuators are used due to a number of benefits, such as being inexpensive and small. On smaller robots, it is effective to take advantage of these strengths; however, what electric actuators supply in benefits, they lack in torque and power. Hydraulics on the other hand offer an enormous amount of power and precision, at the cost of extra weight. Considering the heavy components associated with hydraulics, such as the liquid, reservoir and pump, the power from the actuators are necessary to support this extra component weight and in addition, offer an increased payload. An example of this is the robot Comet-III [38] (see Figure 1.15), a demining robot, that makes use of hydraulic actuators for movement. Since COMET-III weighs $1200 \mathrm{~kg}$, it needs the added power and torque that are offered by the hydraulics for support and walking manoeuvres. 


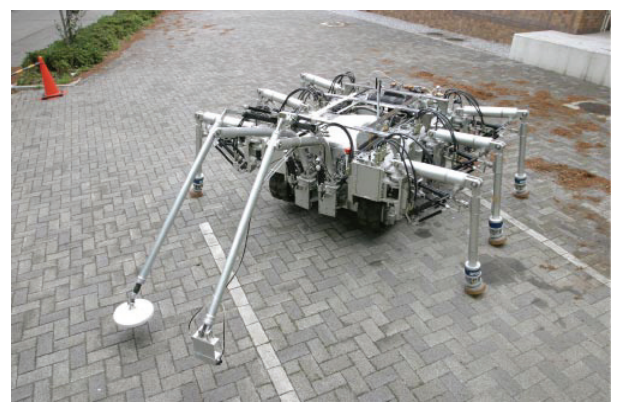

Figure 1.15: COMET-III [39]

Another similar legged hydraulic robot is the TITAN-XI [40] (see Figure 1.16), a construction robot used for drilling purposes. It uses hydraulic actuators to control its four legs for stability as it climbs complex slopes to perform its task. This robot weighs approximately $6800 \mathrm{~kg}$ and thus necessitates hydraulics to provide adequate torque not only for movement up a slope but for the equipment operation as well.

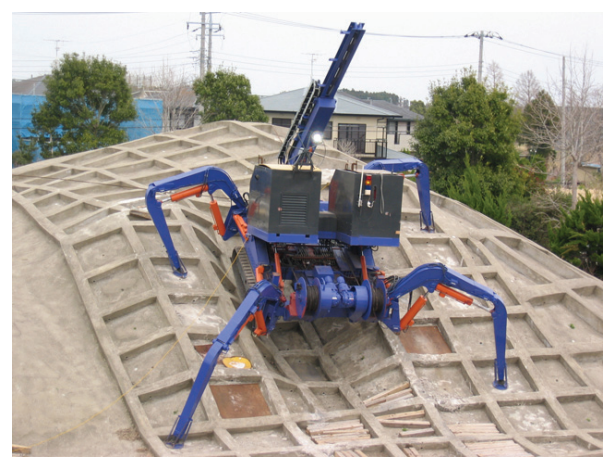

Figure 1.16: TITAN-XI [41]

\subsection{Micro Hydraulic Toolkit (MHT)}

Though the concept of quadruped hybrid robots is not new, making use of hydraulics in combination with this style of locomotion provides the ability to design a more robust robot. The Micro Hydraulic Toolkit (MHT) [42-45] (see Figure 1.17), developed by Defence Research and Development Canada (DRDC) - Suffield, is an example of such a robot. This reconfigurable robot was created for use in a military environment in an 
autonomous capacity. All 12 of MHT's actuators are active, where eight are hydraulic and the four wheels are electrically actuated. More details on the MHT's mechanical setup will be presented in Chapter 2 .

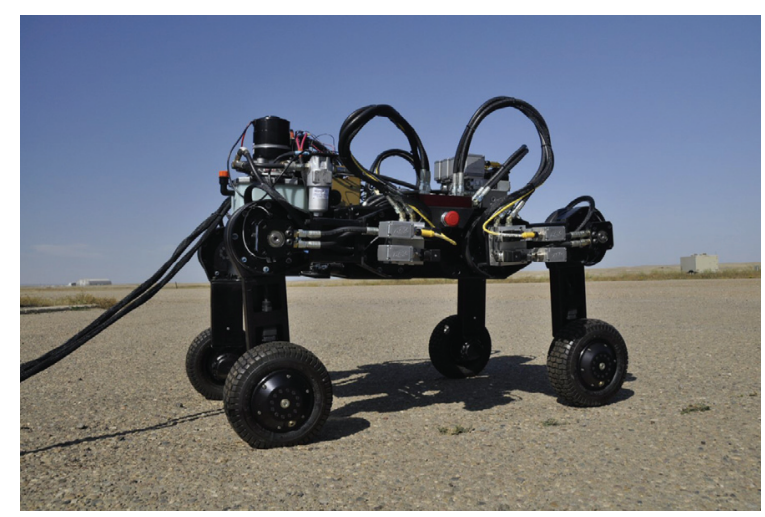

Figure 1.17: Micro Hydraulic Toolkit

As MHT has been constructed for military purposes, the mission statement for the Autonomous Intelligent Systems Section of DRDC is quoted as being:

"To augment soldiers and combat systems by developing and demonstrating practical, cost effective, autonomous intelligent systems capable of completing military missions in complex operating environments" [42]

Thus, the goal of MHT's design is to overcome complex terrain environments while managing vehicle stability in the process. MHT's size and hydraulic actuators provide an increased advantage over obstacles that might otherwise deter a smaller robot with electric actuators.

\subsubsection{Previous Work on MHT}

Considering MHT's complexity, research has been conducted to analyze its stability by comparing several different stability measures [42]. In addition to this, a mapping was created to show the change in MHT's stable platform velocities when encountering a 
step-like obstruction during wheeled motion. Analysis has also been carried out to determine MHT's kinematic range of motion [43] and a fuzzy controller has been proposed for low-level control, based on simulation results [45]. Finally, a linear control strategy was attempted in simulation [44] with unsuccessful results. The motion attempted in that paper will be addressed further in Chapter 3 .

\subsection{Thesis organization}

In this thesis, we develop a new control method for MHT by prescribing trajectory and posture parameters. The process in which this controller functions is with minimal sensing capability and focuses solely on simulation results. Furthermore, an optimization procedure is detailed to create the necessary posture inputs to reduce the static moments, due to gravity, on the hip and knee actuators. Chapter 2 outlines MHT's physical system, detailing several of its key components. In addition to this, it explains both the preliminary simulation model and professional simulation model used for testing during the course of research. Chapter 3 characterizes the system by defining its mobility and actuation redundancy. Furthermore, this chapter explains the control theory based on MHT's inverse kinematics, along with the control inputs and outputs necessary to define the robot's trajectory and posture along with several of the results achieved. Chapter 4 describes a simple optimization technique and the prescribed objective function to calculate the set of posture parameters for the controller. Finally, chapter 5 will briefly conclude the thesis with the work covered and new possible directions to research for advancing MHT's capabilities. 


\subsection{Summary}

As in any project, it is important to use past work to limit possible errors and maximize progress efficiency. This chapter outlined several robot designs with a similar to MHT's mechanical configuration and introduced the goal of this robot within a real world environment. Military scenarios are not only dangerous, but can be physically draining on human soldiers. Therefore, research into more efficient methods of addressing a modern day military environment through autonomous robotics is an important aspect to consider. 


\section{Chapter 2: Micro Hydraulic Toolkit (MHT)}

\subsection{Introduction}

This chapter details MHT's physical system and outlines the dynamics models employed in producing simulation results. MHT was constructed and is currently located at DRDC in Medicine Hat. Therefore, having access to a high-fidelity model of the platform was necessary for sufficient progress to be made. Using simulation models provided helpful insight into addressing control design and implementation on the actual MHT. Prior to developing a controller for MHT, several modeling software was considered to construct a proper dynamics model for effective testing. The modeling software investigated were Matlab’s SimMechanics, MSC.ADAMS and LMS.Virtual.Lab. In the end, the presented results were mainly obtained through the use of LMS.Virtual.Lab.

MHT is a quadruped robot with 12 actuated degrees of freedom which consists of eight hydraulic actuators, located at the hips and knees, and four electric motors driving the wheels. It weighs approximately $145.9 \mathrm{~kg}$ and measures about a meter cubed when in the stance shown in Figure 2.1. 


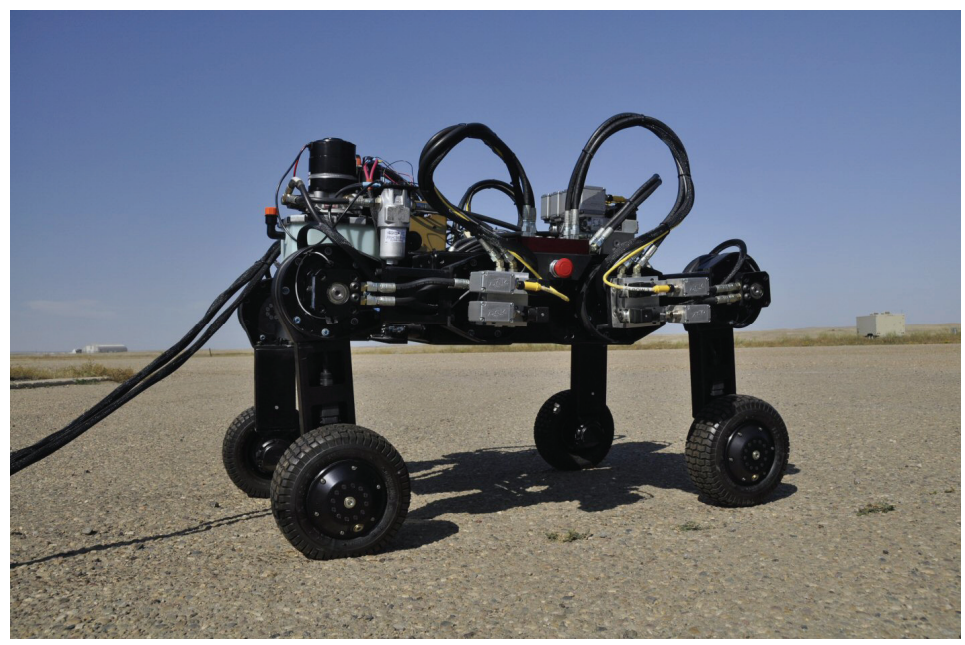

Figure 2.1: MHT neutral position

\subsection{MHT Components}

The MHT robot is a complex system with many components, as described in [42]. The chassis is made up of aluminum and holds the batteries, control electronics, hydraulic hip motors and valves. The four leg assemblies are symmetrically attached to the chassis about the sagittal and coronal plane. The aluminum housing and the aforementioned components weigh approximately $75.2 \mathrm{~kg}$, nearly half of MHT's total weight. The aluminum frame itself only weighs $10 \mathrm{~kg}$.

The accumulator is located at what is considered the front of MHT. It weighs $16.8 \mathrm{~kg}$ and is a container for the pressurized hydraulic liquid, used for driving the hip and knee actuators. The pump/motor/reservoir assembly is located at the rear of MHT and weighs approximately $15.9 \mathrm{~kg}$. This collection of components store and propel the hydraulic liquid that drives the actuators of the hydraulic system. 
The leg assemblies each consist of a femur, knee, tibia and wheel assembly. The length and weight of the femur is $0.315 \mathrm{~m}$ and $0.3 \mathrm{~kg}$, respectively. The knee assembly joins the femur and tibia segments and weighs $4.9 \mathrm{~kg}$. The tibia connects the knee to the wheel assembly with a length and weight of $0.377 \mathrm{~m}$ and $0.5 \mathrm{~kg}$, respectively. Finally, the wheel assembly weighs $4.2 \mathrm{~kg}$ and has a radius of $0.127 \mathrm{~m}$. The motor driving the wheel is electric with a voltage input range between \pm 10 volts. The positive and negative voltages correspond to the forward and reverse direction of the wheel. A summary of the location, weight and dimensional information for the main components is given in Figure 2.3 and Table 2.1. Note that the lengths of the tibia and femur refer to the distance between the actuator axes of rotation associated with that link. Figure 2.2 displays several of MHT's dimensions.

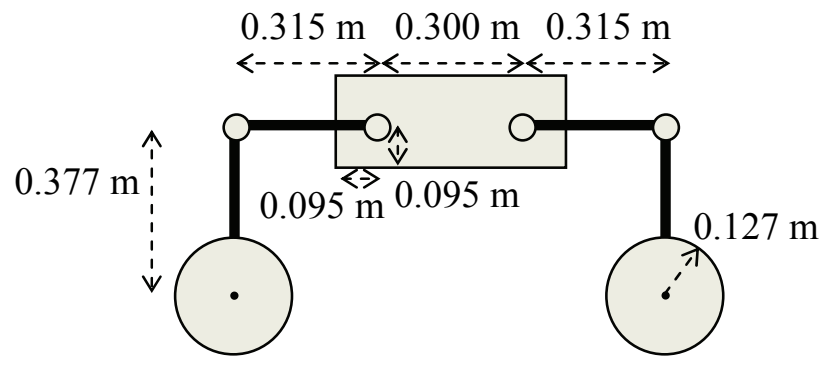

(a)

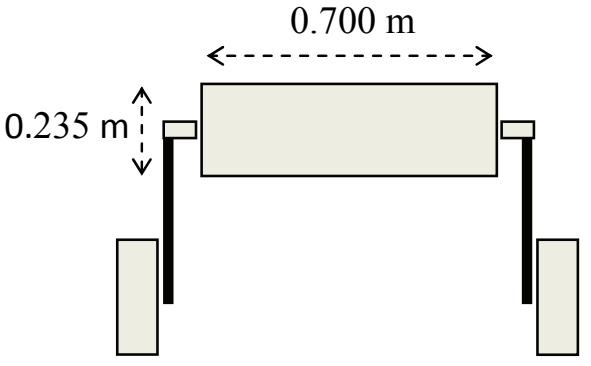

(b)

Figure 2.2: MHT dimensions (a) Side view (b) Front view

The hip and knee actuators can be fastened in $22.5^{\circ}$ increments, allowing re-assembly if necessary. Each rotary hydraulic actuator has a $90^{\circ}$ range of motion. Figure 2.1 illustrates MHT's neutral position, which was chosen as the starting position for each manoeuvre in order to maximize the end-effector reach and height [43]. In this posture, the hip actuator and corresponding femur are at an angle of zero degrees with respect to the platform frame. The knee actuator position and corresponding tibia link are fastened at $90^{\circ}$ with 
respect to the femur. Each hydraulic actuator has a full spool stroke of $\pm 1 \mathrm{~cm}$ from its fastened position, where a full stroke in either direction corresponds to a rotation of \pm 45 degrees at the joint.

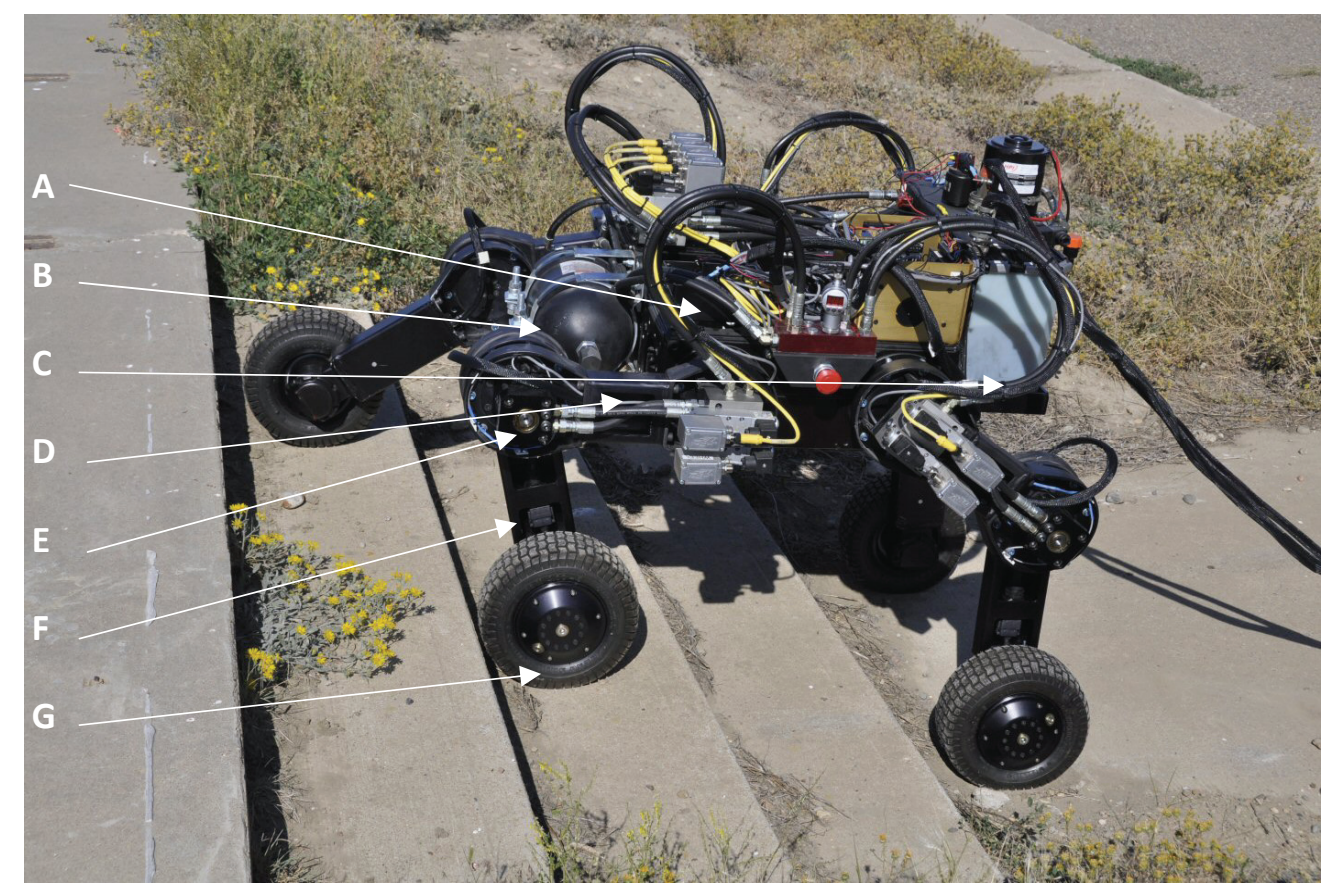

Figure 2.3: Micro Hydraulic Toolkit main components

\begin{tabular}{|c|c|c|c|}
\hline Reference & Component & Weight (kg) & Dimension \\
\hline A & Chassis & 75.2 & $\begin{array}{c}\text { Length: } 0.7 \mathrm{~m} \\
\text { Width: } 0.5 \mathrm{~m} \\
\text { Height: } 0.23 \mathrm{~m} \\
\text { Hip Separation: } 0.3 \mathrm{~m}\end{array}$ \\
\hline B & Accumulator & 16.8 & $\begin{array}{c}\text { Length: } 0.450 \mathrm{~m} \\
\text { Diameter: } 0.180 \mathrm{~m}\end{array}$ \\
\hline $\mathrm{C}$ & Pump/Motor/Reservoir & 15.9 & $\begin{array}{l}\text { Length: } 0.2032 \mathrm{~m} \\
\text { Width: } 0.2032 \mathrm{~m} \\
\text { Height: } 0.457 \mathrm{~m}\end{array}$ \\
\hline $\mathrm{D}$ & Femur & 0.3 & Length: $0.315 \mathrm{~m}$ \\
\hline $\mathrm{E}$ & Knee assembly & 4.9 & \\
\hline $\mathrm{F}$ & Tibia & 0.5 & Length: $0.377 \mathrm{~m}$ \\
\hline G & Wheel assembly & 4.2 & Diameter: $0.254 \mathrm{~m}$ \\
\hline
\end{tabular}

Table 2.1: Summary of components 
The internal interoceptive sensing capability of MHT allows feedback of the chassis' roll, pitch, yaw angles and angular velocity, as well as the chassis' linear acceleration in the $\mathrm{x}$, $\mathrm{y}$ and $\mathrm{z}$ direction. The sensors also include information about the angular position of the hydraulic actuators, as well as the angular rate of all 12 actuated joints [42].

\subsection{SimMechanics and MSC.ADAMS Model}

Several dynamic simulation tools were investigated, initially for the purpose of creating a basic simulation model of the MHT, excluding detailed modelling of the actuators. The objective for creating this simplified model was to test our initial understanding of the system's kinematics, actuation and initial controller design ideas, without having to be concerned with the complexities of hydraulic actuation. The first simulation software considered was Matlab's SimMechanics. An obvious advantage of this software was its immediate compatibility with Matlab's Simulink, the design tool for the controller itself. However, upon further investigation into the software, it was concluded that it could not be applied to MHT's case as a simulation tool. Though user-friendly, SimMechanics lacked the capability to properly model rolling wheel-ground contacts, a crucial aspect of MHT's motion.

Development of the dynamics model was then pursued with the software MSC.ADAMS, an effective multibody dynamics modelling tool. With the supplied information on MHT's geometry and inertial properties of the main components (see Appendix A), a sufficient and accurate model was created to allow initial testing of the controller design proposed in Chapter 3. Wheel-ground contacts were defined using ADAMS' built-in 
contact modelling capabilities, along with the mass and inertia characteristics of MHT's chassis and legs. As no attempt was made to model the hydraulic actuators in ADAMS, the simulation model's inputs were defined as joint rates. A snapshot of the MSC.ADAMS model of MHT is shown in Figure 2.4.

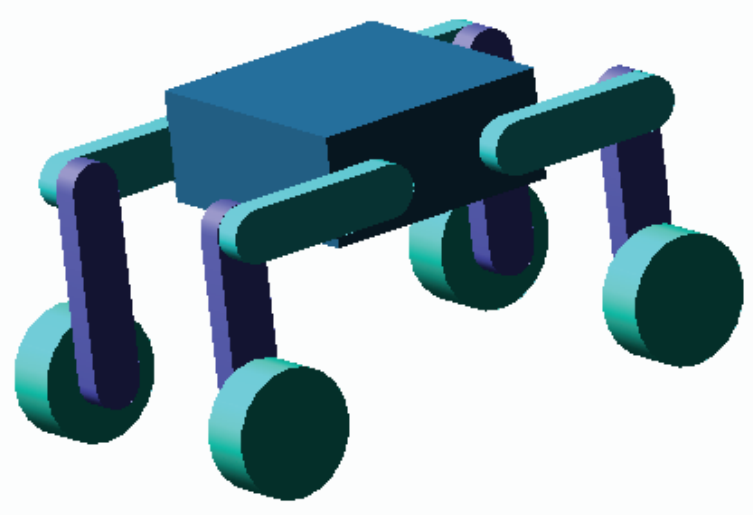

Figure 2.4: MSC.ADAMS MHT snapshot

\subsection{LMS Virtual Lab Model}

The foremost dynamics model, when considering the results relative to this thesis, is the one developed in the software LMS.Virtual.Lab. This high-fidelity three dimensional model of MHT was created by engineers at LMS as part of a subcontract from DRDC Suffield to effectively simulate the actual MHT. The LMS model incorporates significantly more detail compared to the MSC.ADAMS model developed at McGill, as shown in Figure 2.5. It has also been modelled with all the sensors of the actual robot (see Section 2.2) to accommodate any necessary feedback requirements of an assigned controller. 


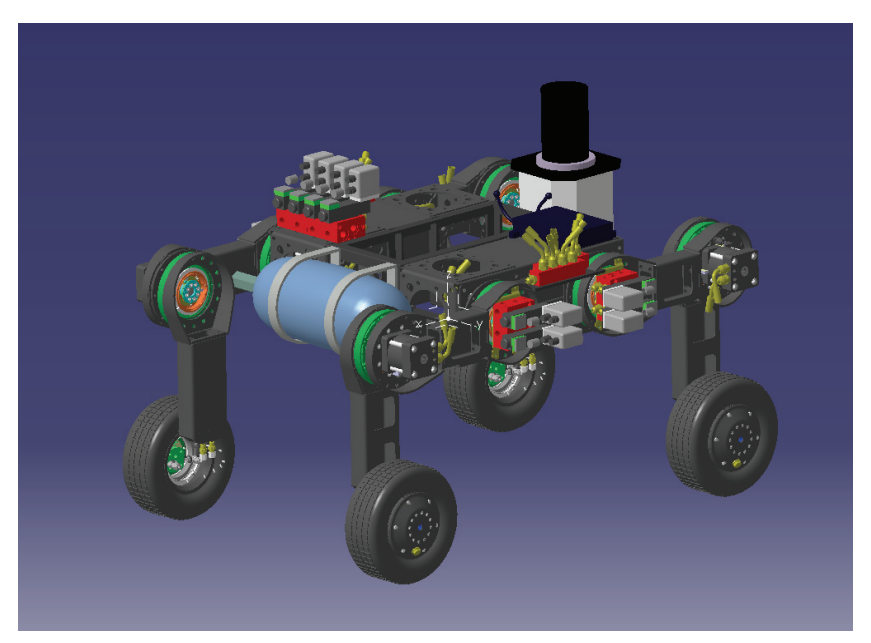

Figure 2.5: LMS MHT snapshot

As opposed to the MSC.ADAMS model, both the hydraulic and electric actuators are incorporated into the LMS model dynamics. The hydraulic actuators for all the hip and knee joints are identical and their specifications can be seen in Table 2.2. The actuator model is based on the assumption that the spool displacement and the input voltage have a direct linear relationship. This relationship is shown in Figure 2.6 with saturation at the $\pm 10 \mathrm{~V}[42]$. The voltage inputs to the LMS model are derived from the controller developed in Matlab's Simulink software as will be described in Chapter 3.

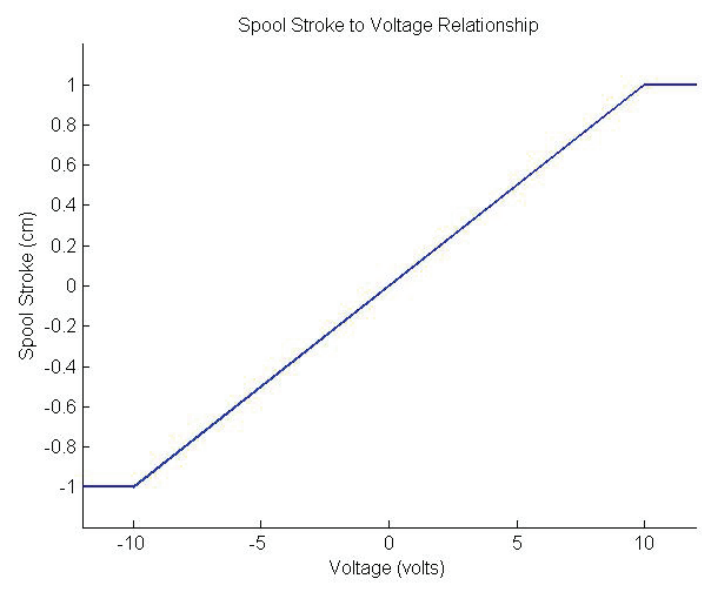

Figure 2.6: Spool stroke to voltage input relationship [42] 


\begin{tabular}{|l|l|}
\hline Type: & Modified double vane \\
\hline Torque: & $474.54 \mathrm{Nm} @ 19856.9 \mathrm{kPa}$ \\
\hline Displacement/Revolution: & $49.98 \mathrm{~cm}^{3} / 100^{\circ}$ \\
\hline Gear Ratio: & 1 (assumed) \\
\hline Efficiency: & 1 (assumed) \\
\hline Line diameter: & $0.635 \mathrm{~cm}$ \\
\hline Valve Coefficient: & $1.0 \times 10^{-5}$ (approximate) \\
\hline & Fluid Properties \\
\hline Fluid: & Shell Tellus T46 \\
\hline Kinematic viscosity: & $46 \mathrm{cST} @ 40^{\circ} \mathrm{C}$ \\
\hline Dynamic viscosity: & $0.0402 \mathrm{Ns} / \mathrm{m}^{2}$ \\
\hline Bulk Modulus: & $1378951.4 \mathrm{kPa}$ (assumed) \\
\hline Fluid Density: & $0.873 \mathrm{~kg} / 1$ \\
\hline
\end{tabular}

Table 2.2: Rotary hydraulic actuator specifications

The positive rotation of each joint angle, as used in LMS, is defined in Figure 2.7 for the hip, knee and wheel joints of MHT. In chapter 3, the sign convention of each joint rotation will be adjusted to follow the right hand rule convention, with respect to the platform frame attached to the chassis of MHT.

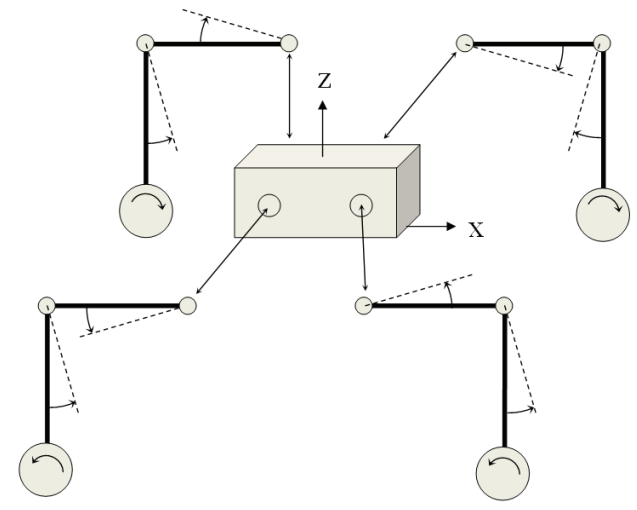

Figure 2.7: LMS model joint direction due to positive voltage input 


\subsection{Summary}

As discussed in this chapter, MHT is a complex reconfigurable robot with significant actuation. The hydraulic actuators of this quadruped provide complications in terms of developing the dynamics model of the system and not all off-the-shelf simulation tools have the same capabilities to model this system. For this reason, using a high-fidelity model offers an effective means of controller design. Though MSC.ADAMS showed promise, LMS provided the highest level of detail for the necessary application of the research conducted. 


\section{Chapter 3: Inverse Kinematics Controller}

\subsection{Introduction}

This chapter outlines the design and implementation of a kinematic controller for the Micro Hydraulic Toolkit (MHT) using velocity kinematic relations for the platform. The controller in question is largely based on that designed for the Hylos robot [35], though in Section 3.6.2, a modification is incorporated for improved posture control. The primary objective of this controller is to affect the desired motion and posture of the MHT in an effective manner on variable terrain. The control method addresses MHT's posture and trajectory; however, the trajectory control implemented in this thesis has no feedback. Previous work with MHT [44] involved controlling each actuator separately without coordinated actuation; however, this resulted in actuator overload and posture failure.

Initial testing of the proposed controller was implemented in simulation, in conjunction with the dynamics model of MHT in MSC.ADAMS, as introduced in Chapter 2. This phase served to establish the reliability of the preliminary control algorithm. Following this, further testing was conducted using the LMS.Virtual.Lab dynamics model. The results presented in this thesis are based on simulation trials which stem from a combination of Matlab's Simulink software, where the controller resides, and the aforementioned models. These results demonstrate the controller's ability to track the desired posture inputs and as shown in Chapter 4, to adapt to varying terrain. The 
trajectory control is considered to be an open-loop controller due to the lack of trajectory sensory information on the robot.

As explained in Chapter 2, the control inputs to MHT are voltage signals which are assumed to directly affect the hydraulic piston position driving the hip and knee actuators. The wheels are driven by electric actuators, whereby positive and negative voltages drive the wheels either forward or reverse. The applied control method determines the actuator voltages necessary for MHT to attain a desired posture and trajectory. The basic idea for the controller is to first define the necessary joint rates, based on the kinematics of MHT, which are then used as desired set points for a PD control law.

In the following section, we will present MHT's kinematic model and show the appropriate coordinate frames. Section 3.3 will demonstrate the elements of MHT's mobility that classify it as a redundantly actuated vehicle. Section 3.4 consists of an overview of the control methodology. Section 3.5 describes the differential kinematics model, which provides the foundation for the controller, while section 3.6 describes the modifications made relative to the original methodology in [36]. Section 3.7 details the simulation results obtained for posture tasks assigned to MHT and finally, section 3.8 will summarize the chapter. 


\subsection{Parameter Definition}

Prior to specifying all the features of the proposed controller, it is important to define the relevant parameters of the kinematic model. The kinematic parameters of the entire system are shown in Figures 3.1 and 3.2, where $\mathfrak{I}_{I}$ is taken as the ground inertial frame and $\mathfrak{I}_{\mathrm{P}}$ is the platform fixed frame.

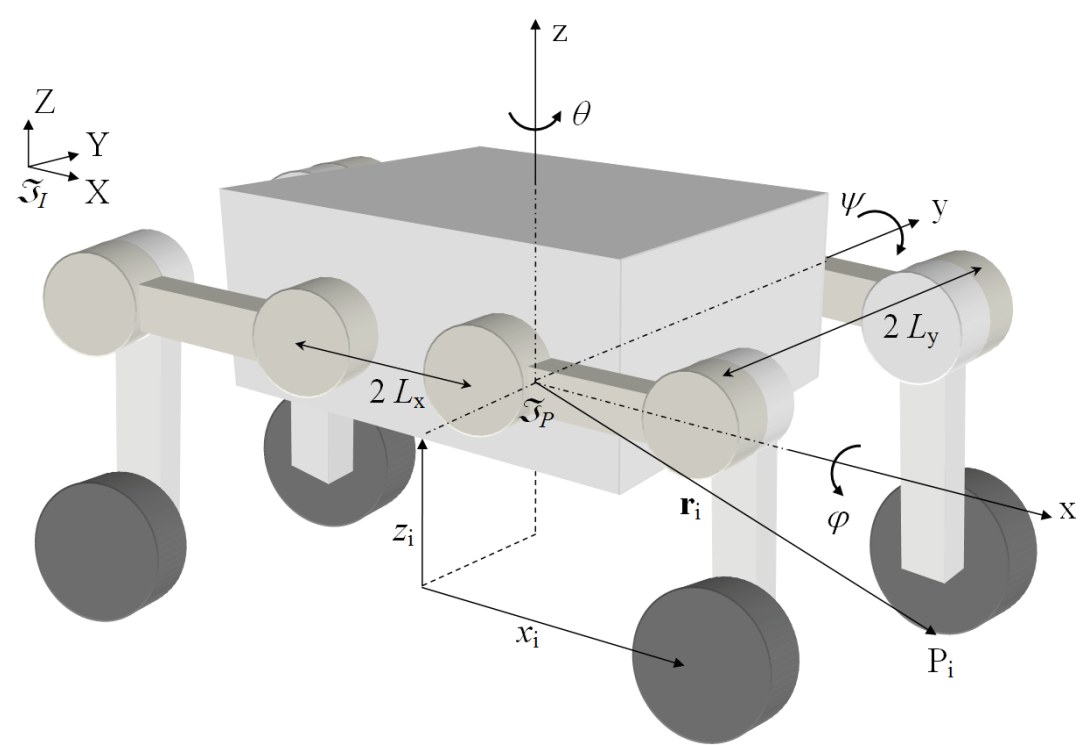

Figure 3.1: MHT platform configuration

In Figure 3.1, $\varphi$ is the roll, $\psi$ is the pitch, $\theta$ is the yaw and $z$ is the relative height, measured as the average $z$-distance from all wheel contact points to the body-fixed frame origin, as defined by equation (3.1). Furthermore, $x_{i}$ and $z_{i}$ represent the distance from the platform body-fixed frame to the center of the $i^{\text {th }}$ wheel along the the platform frame's $x$ and $z$ axis, respectively. Finally, $\mathbf{r}_{i}$ corresponds to the vector from the body-fixed frame origin to the $i^{\text {th }}$ wheel contact point.

$$
z=\frac{\sum r_{z_{i}}}{4} \quad, i=1,2,3,4
$$

Ideally, the origin of the body-fixed frame should coincide with the center of mass of the robot, based on the necessary calculations for the controller. However, in the case of 
MHT, the center of mass will fluctuate depending on its posture, as was described by Beckman et al. [42]. Therefore, for our purposes, considering most of MHT's mass resides within the chassis and its housed components, the frame attached to MHT, $\mathfrak{I}_{\mathrm{P}}$, will be assumed to be fixed to the geometric center of the base of the chassis. In the neutral position, shown in Figure 3.1, the frame, $\mathfrak{I}_{\mathrm{P}}$, will measure $0.409 \mathrm{~m}$ above the contact point of the wheels on level terrain. The kinematic parameters of an individual leg are shown in more detail in Figure 3.2, where $\alpha_{i}$ is the angle of the hip joint, $\beta_{i}$ is the angle of the knee joint and $\omega_{i}$ is the wheel rate, $L_{f}$ is the length of the femur, $L_{t}$ is the length of the tibia and $r_{w}$ is the radius of the wheel. The dotted lines represent the hip and knee joint configurations for which $\alpha$ or $\beta$ are equal to zero; the configuration of MHT when $\alpha=\beta=0$ for all legs will be referred to as MHT's neutral position, as previously mentioned.

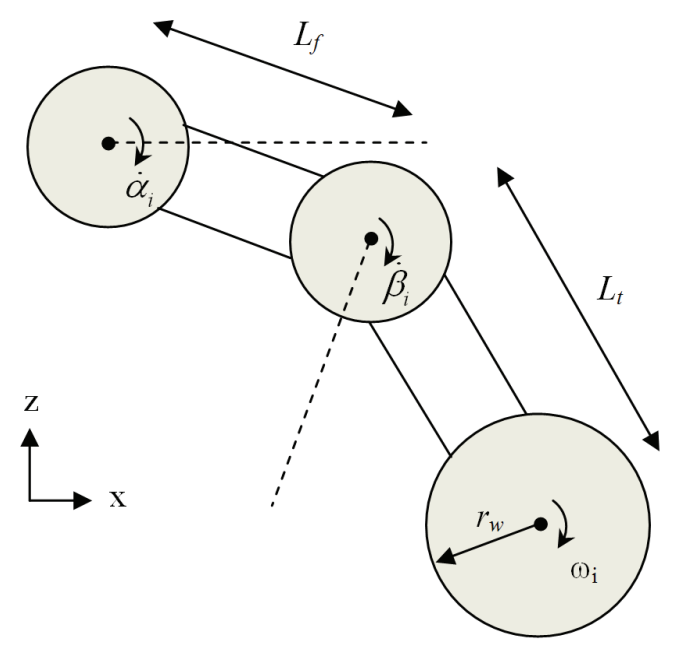

Figure 3.2: MHT leg parameters 


\subsection{Mobility}

Calculating the mobility of MHT will contribute to determining the minimum necessary operational parameters for controlling the system. The mobility, $m$, is first defined using the Chebychev-Grüebler-Kutzbach equation [46]:

$$
m=\sum_{i=1}^{j} f_{i}-6(j-b+1)
$$

where $f_{i}$ is the degree of freedom of the $i^{\text {th }}$ joint, $j$ is the number of joints and $b$ is the number of bodies in the mechanical system in question.

In applying Equation (3.2) to compute the mobility of MHT, we define the contact point of each wheel as a three degree of freedom spherical joint, where it is assumed that the wheel are undergoing ideal rolling without slipping (nonholonomic constraint) ${ }^{1}$ [35]. In making this assumption, we are determining the instantaneous (or internal) mobility of MHT [47], whereby the terrain topology will not be taken into account. All other joints of the platform (hip, knee, wheel) are one degree of freedom joints. MHT has 14 bodies including the vehicle body and the ground and 16 joints, where four of the joints represent the contact points of each wheel. Therefore, for the configurations of the robot when all legs are in contact with the ground (flat or otherwise), the Chebychev-GrüeblerKutzbach equation results in:

$$
m=[(12 \times 1)+(4 \times 3)]-6(16-14+1)=6
$$

\footnotetext{
${ }^{1}$ The nonholonomic constraint prevents the translational motion of the contact point in the plane of the ground, which combined with the normal constraint results in three translational constraints on the contact joint.
} 
The issue that arises in using the Chebychev-Grüebler-Kutzbach equation, is that it does not consider the dependency among the kinematic constraints [35]. The general constraints of the system can be set up as follows:

$$
\mathbf{A} \dot{\mathbf{q}}=\mathbf{0}
$$

As described by Grand et al. [35], the matrix A can be formulated from Eq. (3.23), to be developed in Section 3.5 of the thesis, producing the following form:

$$
\mathbf{A}=\left[\begin{array}{ccccc}
\mathbf{J}_{1} & \mathbf{0} & \mathbf{0} & \mathbf{0} & \mathbf{L}_{1} \\
\mathbf{0} & \mathbf{J}_{2} & \mathbf{0} & \mathbf{0} & \mathbf{L}_{2} \\
\mathbf{0} & \mathbf{0} & \mathbf{J}_{3} & \mathbf{0} & \mathbf{L}_{3} \\
\mathbf{0} & \mathbf{0} & \mathbf{0} & \mathbf{J}_{4} & \mathbf{L}_{4}
\end{array}\right]
$$

where $\mathbf{J}_{i}$ and $\mathbf{L}_{i}$ are the Jacobian and Locomotion matrices for $i=1,2,3,4$ (see Section 3.5 for details). However, independence between all the constraint equations may not necessarily be the case. Therefore, another method of determining the mobility of MHT is to disregard the dependent equations of the system and thus evaluate the mobility of the system according to $[35,48]$.

$$
m=\operatorname{dim}(\dot{\mathbf{q}})-\operatorname{rank}(\mathbf{A})
$$

It is important to note that the mobility of this system is subject to change with modifications in MHT's configuration. In applying Equation (3.3) to MHT, the mobility evaluated was eight for the neutral configuration, shown in Figure 3.3, while it was computed as seven for the standing configuration shown in Figure 3.4. Similar phenomenon was noticed by Grand et al. [35] when calculating the mobility for the robot Hylos, which was attributed to the partial internal mobility. Only in the exact neutral 
position was MHT's mobility calculated to be eight, while in any other configuration, it was seven.

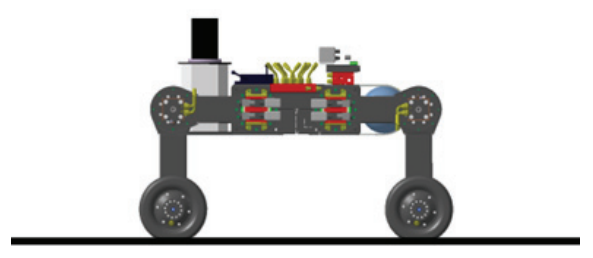

Figure 3.3: Neutral position $(m=8)$

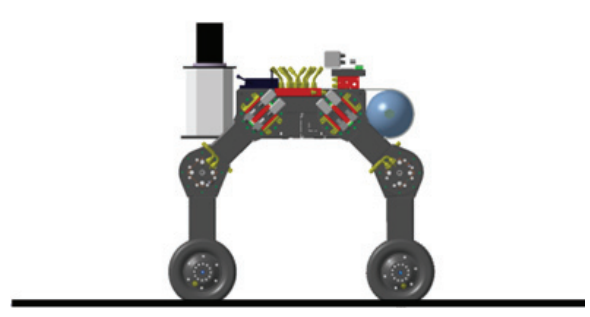

Figure 3.4: Standing position $(m=7)$

Furthermore, MHT can be classified as redundantly actuated, where Equation (3.4) can be applied to calculate its actuation redundancy, $r$, as described by Nahon [49].

$$
r=g_{a}-m
$$

where $g_{\mathrm{a}}$ refers to the number of installed actuators and $m$ is the mobility of the system. Thus, based on this calculation, in the majority of postures, MHT's redundancy is calculated to be five, whereas during it neutral position, its redundancy is four.

Therefore, according to mobility analysis, a minimum of seven inputs are necessary to pilot the MHT platform. However, the goal of the controller proposed in Section 3.4 is to exert greater posture control with regards to independent motion of the legs. For this reason, more operational inputs will be selected than the calculated mobility of the system. This will allow control over both the wheelbase and stepping manoeuvres, as will be discussed in the following sections. 


\subsection{Controller Overview}

This section addresses the design of the kinematic controller applied to the MHT platform. This controller would also be suitable for other quadruped wheeled robots with a similar kinematic design. Figure 3.5 shows the general schematic for the control strategy.

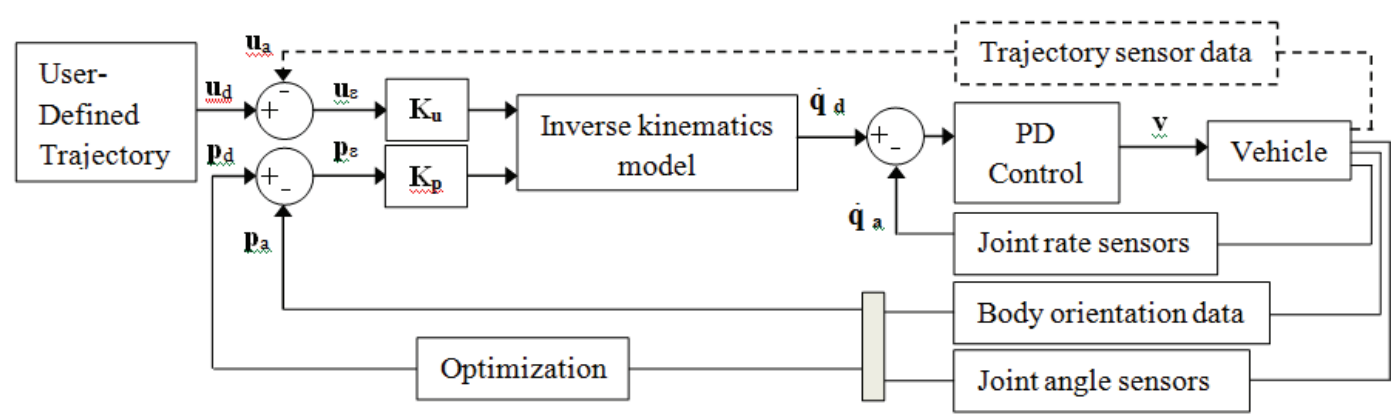

Figure 3.5: Control schematic

In the above figure, $\mathbf{p}_{\mathrm{d}}$ is the desired posture, $\mathbf{p}_{\mathrm{a}}$ is the actual posture, $\mathbf{u}_{\mathrm{d}}$ is the desired trajectory, $\mathbf{u}_{\mathrm{a}}$ is the actual trajectory, $\dot{\mathbf{q}}_{\mathrm{d}}$ is the desired joint rates, $\dot{\mathbf{q}}_{\mathrm{a}}$ is the actual joint rates and $\mathbf{v}$ is the voltage signal. The controller input defined in this section is separated into two types, the posture inputs and trajectory inputs, represented by a vector and each having desired and actual values as defined below:

Posture Inputs:

$$
\begin{aligned}
\mathbf{p}_{\mathrm{d}} & =\left[\begin{array}{lllllllllll}
\varphi_{d} & \psi_{d} & z_{d} & x_{1_{d}} & x_{2_{d}} & x_{3_{d}} & x_{4_{d}} & z_{1_{d}} & z_{2_{d}} & z_{3_{d}} & z_{4_{d}}
\end{array}\right]^{T} \\
\mathbf{p}_{\mathrm{a}} & =\left[\begin{array}{lllllllllll}
\varphi_{a} & \psi_{a} & z_{a} & x_{1_{a}} & x_{2_{a}} & x_{3_{a}} & x_{4_{a}} & z_{1_{a}} & z_{2_{a}} & z_{3_{a}} & z_{4_{a}}
\end{array}\right]^{T}
\end{aligned}
$$

Trajectory Inputs:

$$
\begin{aligned}
& \mathbf{u}_{\mathrm{d}}=\left[\begin{array}{lll}
x_{d} & y_{d} & \theta_{d}
\end{array}\right]^{T} \\
& \mathbf{u}_{\mathrm{a}}=\left[\begin{array}{lll}
x_{a} & y_{a} & \theta_{a}
\end{array}\right]^{T}
\end{aligned}
$$


The values within these vectors are defined in Figures 3.1 and 3.2, where subscripts one through four refer to each leg. In the scope of the present thesis, the sensor values for joint angles, angular rates and platform orientation angles are assumed to be exact. The posture and trajectory are prescribed by the user and can vary depending on the task expected of MHT. The focus of this controller will remain on closed loop posture control to adapt MHT's kinematic configuration to perform manoeuvres and smoothly overcome terrain obstacles. The trajectory control will act as open loop control method to implement simple drive commands, such as moving forward and backward.

The output of the inverse kinematics model (see Figure 3.5) represents the joint rates of each individual joint actuator:

$$
\begin{aligned}
& \dot{\mathbf{q}}_{i_{d}}=\left[\begin{array}{lll}
\dot{\alpha}_{i_{d}} & \dot{\beta}_{i_{d}} & \omega_{i_{d}}
\end{array}\right]^{T} \\
& \dot{\mathbf{q}}_{i_{a}}=\left[\begin{array}{lll}
\dot{\alpha}_{i_{a}} & \dot{\beta}_{i_{a}} & \omega_{i_{a}}
\end{array}\right]^{T}
\end{aligned}
$$

where $\dot{\mathbf{q}}_{i_{d}}$ are the desired joint rates of the $i^{\text {th }}$ leg and $\dot{\mathbf{q}}_{i_{a}}$ are the actual joint rates of the $i^{\text {th }}$ leg.

Finally, applying a PD control to the joint rate error, we obtain the voltage inputs to the dynamics model of the vehicle. The control law used is defined by Equation (3.11).

$$
V_{j}=K_{P}\left(\dot{q}_{j_{d}}-\dot{q}_{j_{a}}\right)+K_{D} \frac{d}{d t}\left(\dot{q}_{j_{d}}-\dot{q}_{j_{a}}\right)
$$


The variable $V_{j}$ represents the voltage applied to the $j^{\text {th }}$ actcuator, $\dot{q}_{j_{d}}$ is the desired angular rate of the $j^{\text {th }}$ actuator, $\dot{q}_{j_{a}}$ is the actual angular rate of the $j^{\text {th }}$ actuator and $K_{P}$ and $K_{D}$ are the proportional and derivative gains, respectively.

\subsection{Differential Kinematic Model}

The kinematic model is defined using the velocity kinematic relations for the body and articulated legs of MHT. Using velocity addition, the equation for such a system can be established as follows, maintaining all calculations in the platform frame:

$$
\mathbf{v}_{i}=\mathbf{v}_{P_{i} / p}+\mathbf{v}_{P_{i} / l}
$$

where $\mathbf{v}_{i}$ is the sliding velocity at the $i^{\text {th }}$ contact point, $\mathbf{v}_{P i / p}$ is the velocity at the $i^{\text {th }}$ contact point due to platform motion and $\mathbf{v}_{P i / l}$ is the velocity at point $\mathrm{i}$ due to the leg motion.

Figure 3.6 displays the velocity vectors on MHT's leg for an indeterminate terrain.

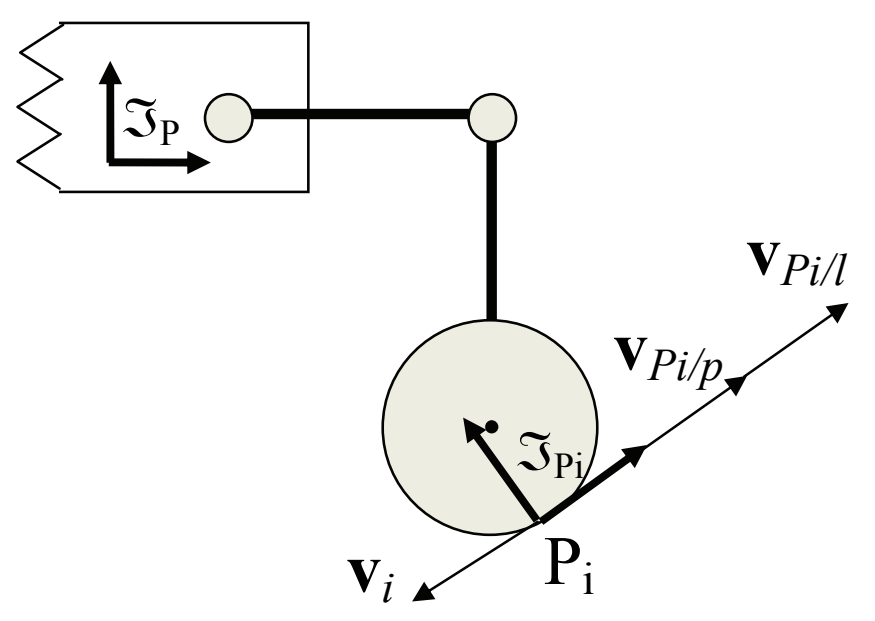

Figure 3.6: Velocity composition of $i^{\text {th }}$ leg

The symbols $\mathfrak{I}_{\mathrm{P}}$ and $\mathfrak{I}_{\mathrm{Pi}}$ refer to the coordinate frames of the platform and contact point, respectively. Under the assumption of rolling with no slipping, we let $\mathbf{v}_{i}=0$, so that:

$$
\mathbf{v}_{P_{i} / p}+\mathbf{v}_{P_{i} / l}=\mathbf{0}
$$


Next, the equation defining the velocity of each contact point due to platform motion with respect to the ground frame is defined as:

$$
\mathbf{v}_{P i / p}=\mathbf{R} \dot{\boldsymbol{\rho}}+\boldsymbol{\omega} \times \mathbf{p}_{i}
$$

where $\mathbf{R}$ is the rotation matrix from the ground frame to the platform frame (Eq. 3.15), $\dot{\boldsymbol{\rho}}$ is the translational velocity of the platform center of mass with respect to the ground frame, $\omega$ is the platform angular velocity defined in the platform frame, while $\mathbf{p}_{i}$ is the vector from the center of mass to the contact point also described in the platform frame. The rotation matrix $\mathbf{R}$ employed in Eq. (3.15) is obtained from the roll, pitch and yaw orientation angles of the body as:

$$
\mathbf{R}=\left[\begin{array}{ccc}
C_{\theta} C_{\psi} & S_{\theta} C_{\psi} & -S_{\psi} \\
-S_{\theta} C_{\varphi}+C_{\theta} S_{\psi} S_{\varphi} & C_{\theta} C_{\varphi}+S_{\theta} S_{\psi} S_{\varphi} & C_{\psi} S_{\varphi} \\
S_{\theta} S_{\varphi}+C_{\theta} S_{\psi} C_{\varphi} & -C_{\theta} S_{\varphi}+S_{\theta} S_{\psi} C_{\varphi} & C_{\psi} C_{\varphi}
\end{array}\right]
$$

To describe the vector $\omega$ as a function of our input parameters [35], we employ:

$$
\boldsymbol{\omega}=\left[\begin{array}{ccc}
1 & 0 & -S_{\psi} \\
0 & C_{\varphi} & -C_{\psi} S_{\varphi} \\
0 & -S_{\varphi} & C_{\psi} C_{\varphi}
\end{array}\right]\left[\begin{array}{c}
\dot{\varphi} \\
\dot{\psi} \\
\dot{\theta}
\end{array}\right]=\mathbf{T}_{\phi} \dot{\phi}
$$

We can now rewrite Eq. (3.14) by making use of:

$$
\boldsymbol{\omega} \times \mathbf{p}_{i}=\mathbf{T}_{\phi} \dot{\phi} \times \mathbf{p}_{i}=-\tilde{\mathbf{p}}_{\mathrm{i}} \mathbf{T}_{\phi} \dot{\phi}
$$

where $\tilde{\mathbf{p}}_{i}$ is the skew-symmetric matrix of the vector $\mathbf{p}_{i}$. Equation (3.14) is rewritten as:

$$
\mathbf{v}_{P_{i} / p}=\mathbf{L}_{i} \mathbf{v}_{p}
$$

where $\mathbf{L}_{i}=\left[\begin{array}{ll}\mathbf{R} & -\tilde{\mathbf{p}}_{i} \mathbf{T}_{\phi}\end{array}\right]$ and $\mathbf{v}_{p}=\left[\begin{array}{c}\dot{\boldsymbol{\rho}} \\ \dot{\boldsymbol{\phi}}\end{array}\right]$. 
In the above, we introduced the so called locomotion matrix [35], which when multiplied by the time-derivative of the selected posture and trajectory parameters, grouped in the twist vector $\mathbf{v}_{p}$, determines the velocity of the contact point due to platform motion. Furthermore, the twist vector is parameterized using the selection matrices to attain the necessary form:

$$
\mathbf{v}_{p}=\mathbf{C}_{t} \dot{\mathbf{u}}+\mathbf{C}_{p} \dot{\mathbf{p}}=\left[\begin{array}{llllll}
\dot{x} & \dot{y} & \dot{z} & \dot{\varphi} & \dot{\psi} & \dot{\theta}
\end{array}\right]^{T}
$$

where the matrices $\mathbf{C}_{t}$ and $\mathbf{C}_{p}$ are the selection matrices for the trajectory and posture time-derivative vectors, respectively.

The time derivatives of the posture and trajectory inputs required in Eq. (3.19) are obtained by using a linearization control law, applying the diagonal gain matrices $\mathbf{K}_{p}$ and $\mathbf{K}_{\mathrm{t}}$ to the posture and trajectory errors, respectively. The twist vector is parameterized by the time derivatives of both trajectory and posture.

$$
\begin{aligned}
\dot{\mathbf{p}} & =\mathbf{K}_{p} \Delta \mathbf{p} \\
\dot{\mathbf{u}} & =\mathbf{K}_{t} \Delta \mathbf{u}
\end{aligned}
$$

In the above, $\Delta \mathbf{p}$ represents the posture error and $\Delta \mathbf{u}$ is the trajectory error, which implies that the actual posture and trajectory information on the robot must be obtained from the sensors on the robot.

Now that the contact point velocity due to platform motion has been defined with respect to the platform, we employ the classical Jacobian matrix to calculate the velocity of the contact point due to the leg's displacement in the platform frame: 


$$
\mathbf{v}_{P_{i} / l}=\left[\begin{array}{lll}
\mathbf{y}_{1} \times \mathbf{a}_{i} & \mathbf{y}_{2} \times \mathbf{b}_{i} & \mathbf{y}_{3} \times \mathbf{c}_{i}
\end{array}\right] \dot{\mathbf{q}}_{i}=\mathbf{J}_{i} \dot{\mathbf{q}}_{i}
$$

where $\mathbf{y}_{1}, \mathbf{y}_{2}$ and $\mathbf{y}_{3}$ are unit vectors aligned with the axes of rotation for the hip, knee and wheel, respectively, $\mathbf{a}_{i}$ is the position vector from the hip joint to the $i^{\text {th }}$ contact point in the platform frame, $\mathbf{b}_{i}$ is the position vector from the knee to the $i^{\text {th }}$ contact point, $\mathbf{c}_{i}$ is the position vector from the wheel to the $i^{\text {th }}$ contact point and $\dot{\mathbf{q}}_{i}$ contains the $i^{\text {th }}$ leg actuator rates. The Jacobian matrix for leg $\mathrm{i}$ is defined in terms of the hip angles, knee angles, and wheel radius as follows:

$$
\mathbf{J}_{\mathrm{i}}=\left[\begin{array}{ccc}
\mp L_{f} \sin \left(\alpha_{\mathrm{i}}\right)-L_{t} \cos \left(\alpha_{\mathrm{i}}+\beta_{\mathrm{i}}\right)-r_{w} & -L_{t} \cos \left(\alpha_{\mathrm{i}}+\beta_{\mathrm{i}}\right)-r_{w} & -r_{w} \\
0 & 0 & 0 \\
\mp L_{f} \cos \left(\alpha_{\mathrm{i}}\right)+L_{t} \sin \left(\alpha_{\mathrm{i}}+\beta_{\mathrm{i}}\right) & L_{t} \sin \left(\alpha_{\mathrm{i}}+\beta_{\mathrm{i}}\right) & 0
\end{array}\right]
$$

The sign convention for some of the terms will vary based on wether the Jacobian is defined for the front legs $(+)$ or the rear legs $(-)$. With the calculated locomotion and Jacobian matrices, the joint rates needed to describe the platform twist vector can be determined using Eq. (3.24):

$$
\mathbf{J}_{i} \dot{\mathbf{q}}_{i}+\mathbf{L}_{i} \mathbf{v}_{p}=\mathbf{0} \quad, i=1,2,3,4
$$

Therefore Eq. (3.24) describes the relationship between the $i^{\text {th }}$ leg joint rates and the platform twist. However, it can be seen from Equation (3.23) that the Jacobian has a determinant of zero and thus is singular. The cause of this singularity is due to the fact that each leg can only operate as a planar manipulator, while the Jacobian matrix is defined spatially, thereby considering a dimension that the manipulator may not operate in. Therefore, this will result in an infinite number of possible leg configurations for a single platform twist vector. An example of this is displayed in Figure 3.7, which shows two postures resulting in the same platform twist. However, the support polygons [37], 
for the two configurations are drastically different, affecting the robot's stability. To properly address the issue of multiple solutions, several modifications are made to Equation (3.24) to improve control over the desired postures.
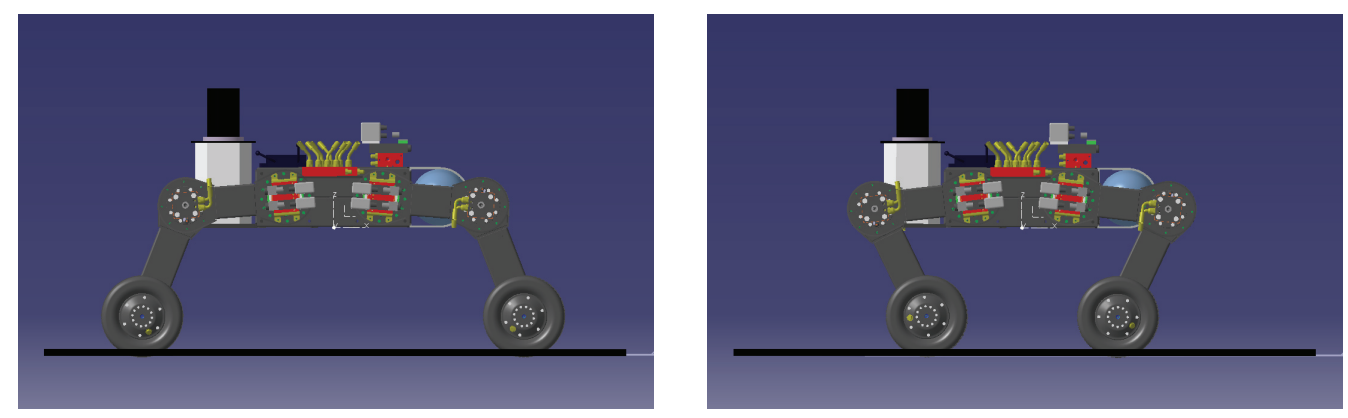

Figure 3.7: Two solutions for the same platform twist

\subsection{Augmented Velocity Model}

The following two subsections describe the changes made to the inverse kinematics formulation of Section 3.5. The first describes the approach used in [35], which is implemented to define the posture and to drive over terrain. The second subsection defines the modifications, mentionned in Section 3.1, to achieve control of the vertical position of the individual wheel for stepping manoeuvres.

\subsubsection{MHT Velocity Model}

As previously mentioned, Equation (3.23) defines a singular Jacobian, leading to an infinite number of solutions to the inverse kinematics problem. To resolve this issue, the equation for the $y$-velocity is removed from the system by multiplying both sides of Eq. (3.24) by a row-eliminating matrix $\mathbf{B}$ :

$$
\mathbf{B}=\left[\begin{array}{lll}
1 & 0 & 0 \\
0 & 0 & 1
\end{array}\right]
$$


In removing the redundant equation from Equation (3.24), we substitute in another equation in its place, parameterized by the added control inputs. Vector $\mathbf{v}_{p}$ is augmented to include the time-derivatives of the $x$-position of the center of the $i^{\text {th }}$ wheel and the adjusted locomotion matrix. Therefore, we introduce:

$$
\begin{aligned}
\tilde{\mathbf{v}}_{p_{i}} & =\left[\begin{array}{ll}
\mathbf{v}_{p} & \dot{x}_{i}
\end{array}\right]^{T} \\
\tilde{\mathbf{L}}_{i} & =\left[\begin{array}{cc}
\mathbf{B L} \mathbf{L}_{i} & \mathbf{0} \\
\mathbf{0} & 1
\end{array}\right]
\end{aligned}
$$

where $\mathbf{B L}_{i}$ is now a 2 × 6 matrix. Corresponding changes need to be made to the Jacobian term in Eq. (3.24). Using the forward kinematics equations, the horizontal velocity $\dot{x}_{i}$ of the wheels can be parameterized as follows:

$$
\dot{x}_{i}=\left(\mp L_{f} \sin \alpha_{i}-L_{t} \cos \left(\alpha_{i}+\beta_{i}\right)\right) \dot{\alpha}_{i}+\left(-L_{t} \cos \left(\alpha_{i}+\beta_{i}\right)\right) \dot{\beta}_{i}
$$

where the sign changes for the front wheels (-) and back wheels $(+)$, respectively. We introduce the augmented Jacobian as:

$$
\tilde{\mathbf{J}}_{i}=\left[\begin{array}{ccc}
\mathbf{B J}_{i} & \\
\mp L_{f} \sin \alpha_{i}-L_{t} \cos \left(\alpha_{i}+\beta_{i}\right) & -L_{t} \sin \left(\alpha_{i}+\beta_{i}\right) & 0
\end{array}\right]
$$

where $\mathbf{B} \mathbf{J}_{\mathrm{i}}$ is a 2 × 3 matrix and the sign convention is chosen depending on wether the Jacobian is for the front legs or rear legs. With the above modifications, solving for the joint rates now offers a unique solution when the modified Jacobian is of full rank. For MHT's case, full rank of the Jacobian matrix is preserved due to the limited range of the actuators; however, this will not be proven here.

The augmented velocity kinematics model for each leg takes the following form:

$$
\dot{\mathbf{q}}_{i}=-\tilde{\mathbf{J}}_{i}^{-1} \tilde{\mathbf{L}}_{i} \tilde{\mathbf{v}}_{p_{i}} \quad, i=1,2,3,4
$$


where $\tilde{\mathbf{J}}_{i}^{-1}$ is the inverse of the augmented Jacobian, $\tilde{\mathbf{L}}_{i}$ is the new locomotion matrix and $\tilde{\mathbf{v}}_{p_{i}}$ includes the platform twist and the horizontal velocities of the wheel centers.

Equation (3.30) provides a set-point, based on the defined gains, of the necessary joint rates for each leg required for a given trajectory and posture. Applying the PD control algorithm defined in Equation (3.11), with appropriate gains, provides the voltages required for the hydraulic actuators to achieve the desired posture and trajectory.

\subsubsection{Stepping Velocity Model}

Though Section 3.6.1 describes an effective controller based on [35], it is limited in only being able to perform manoeuvres where all wheels are in contact with the ground. However, it is important to not only recognize the capability of MHT's legged locomotion, but also to utilize it to its full potential. To this end, an extension to the controller is proposed to take advantage of the walking capability of MHT when necessary. Therefore, this proposed controller can be effectively switch between the controller introduced in Section 3.6.1 and its stepping form.

The modifications for the stepping controller are similar to those described in Section 3.6.1 except that we now add an equation to specify the $z$-position of each wheel relative to the platform frame. The aforementioned equation is as follows:

$$
\dot{z}_{i}=\left( \pm L_{f} \cos \alpha_{i}-L_{t} \sin \left(\alpha_{i}+\beta_{i}\right)\right) \dot{\alpha}_{i}+\left(-L_{t} \sin \left(\alpha_{i}+\beta_{i}\right)\right) \dot{\beta}_{i}
$$

where the sign changes from the front wheels $(+)$ and back wheels $(-)$, respectively. Incorporating Eq. (3.31) into Eq. (3.30) gives the following form: 


$$
\tilde{\mathbf{J}}_{i} \dot{\mathbf{q}}_{i}+\tilde{\mathbf{L}}_{i} \tilde{\mathbf{v}}_{p_{i}}=\mathbf{0} \quad, i=1,2,3,4
$$

Where we redefine the augmented locomotion matrix and Jacobian matrices accordingly

as:

$$
\begin{aligned}
\tilde{\mathbf{J}}_{i} & =\left[\begin{array}{ccc}
\mathbf{B} \mathbf{J}_{i} & \\
\mp L_{f} \sin \alpha_{i}-L_{t} \cos \left(\alpha_{i}+\beta_{i}\right) & -L_{t} \sin \left(\alpha_{i}+\beta_{i}\right) & 0 \\
\pm L_{f} \cos \alpha_{i}-L_{t} \sin \left(\alpha_{i}+\beta_{i}\right) & -L_{t} \sin \left(\alpha_{i}+\beta_{i}\right) & 0
\end{array}\right] \\
\tilde{\mathbf{L}}_{i} & =\left[\begin{array}{ccc}
\mathbf{B} \mathbf{L}_{i} & 0 & 0 \\
0 & 1 & 0 \\
0 & 0 & 1
\end{array}\right] \text { and } \tilde{\mathbf{v}}_{p_{i}}=\left[\begin{array}{c}
\mathbf{v}_{p} \\
\dot{x}_{i} \\
\dot{z}_{i}
\end{array}\right]^{\mathrm{T}}
\end{aligned}
$$

The resulting system (3.32) now represents an overdetermined system of four equations and three unknowns for each leg. Therefore, in general it will not give a solution because there are too many equations for the number of unknowns. As a result, we employ the Moore-Penrose pseudo-inverse to determine the best approximation (in the least-squares sense) for the joint rates to achieve the desired inputs.

\subsection{Simulation Results}

To verify the effectiveness of the inverse kinematics controller defined in the previous section, several different test cases are investigated in simulation of MHT. The first test case involves conducting a symmetrical motion about the platform's sagittal plane, which was previously attempted by Beckman et al. [44]. The second case consists of MHT driving over a specified uneven terrain, while maintaining a level platform (no roll or pitch). A manoeuvre over uneven terrain demonstrates MHT's use of reconfigurable posture to adapt to an inconsistent landscape. The third case shows the stepping controller's capability to overcome step obstacles or obstacles that cannot be otherwise 
driven over. All of these test cases provide ample evidence that the described controller adds significant posture control versatility.

\subsubsection{Vertical Motion Test Case}

The first test case is an aggressive posture manoeuvre that shows MHT's ability to manipulate its topology while overall, the vehicle remains stationary. Beckman et al. [44] first introduced this motion by controlling each actuator independently. In their paper, a problem was detailed where the actual actuator position deviated from the desired actuator position, causing a runaway issue. This runaway began at the hip actuators, approximately seven seconds into the manoeuvre, as indicated in Figures 3.8 and 3.9.

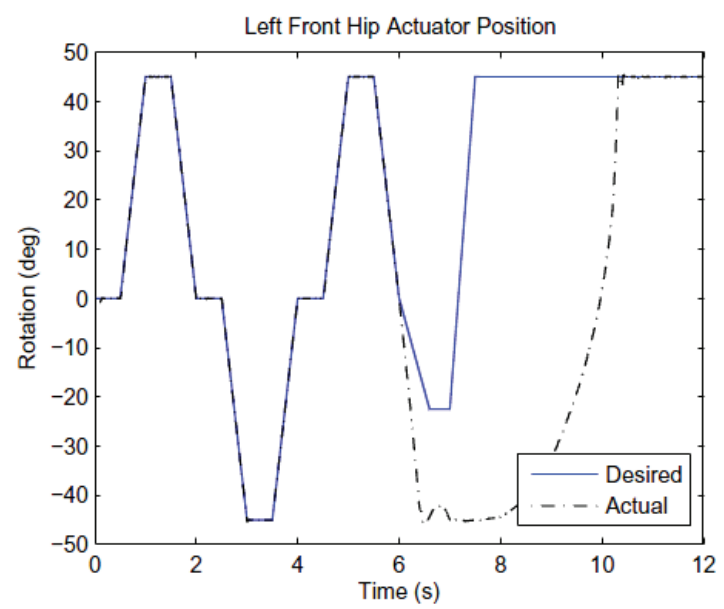

Figure 3.8: Left hip angle runaway [44]

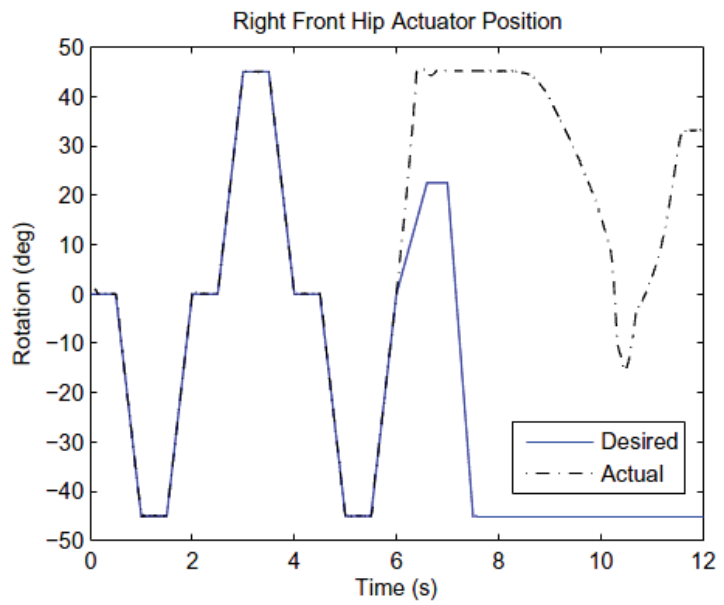

Figure 3.9: Right hip angle runaway [44]

The control strategy undertaken by Beckman et al. used a hand-tuned PD controller based on a simplified mathematical model, applied to each separate actuator. As mentioned in Section 3.6, the newly proposed controller accounts for the kinematics of the platform orientation and leg configurations, which allows for more intuitive posture and trajectory inputs with better results. 
The results shown here will demonstrate the present controller's success in accomplishing this manoeuvre. Figure 3.10 shows the sequential phases of the motion.

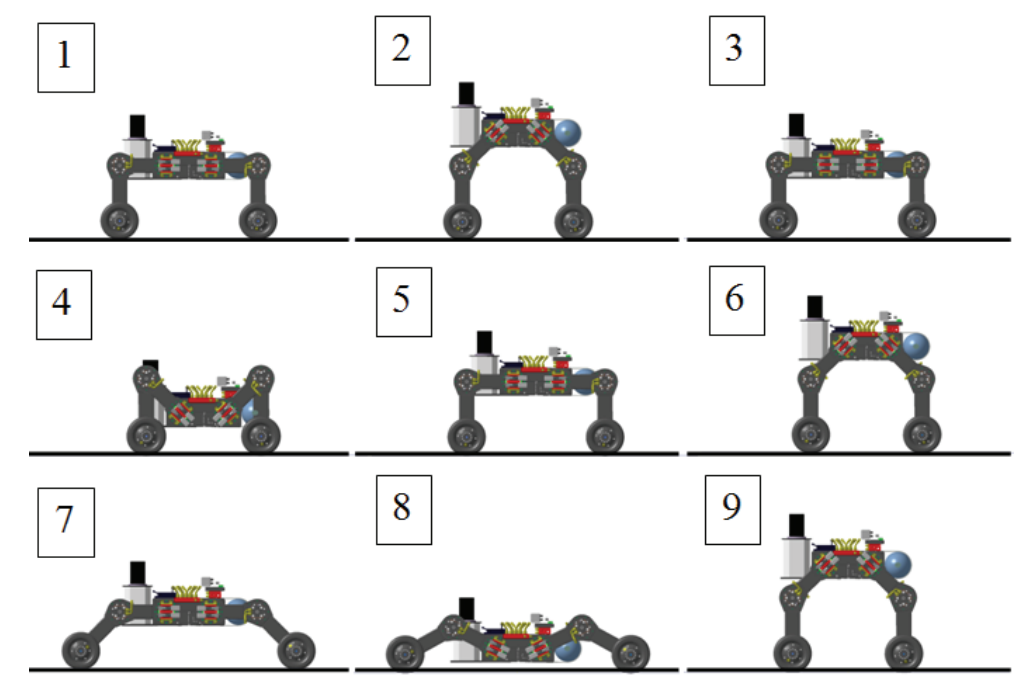

Figure 3.10: Vertical motion snapshots

The motion begins with MHT in its neutral position (phase one), followed by phases two through six displaying MHT's center of mass moving up, back to neutral height, then down and back up again while maintaining a constant wheel base and negligible pitch and roll. Phases seven and eight are where MHT's wheelbase increases to further lower its center of mass and finally phase nine is MHT returning to its final standing up position. The overall time of this manoeuvre is approximately eight seconds, where each phase is maintained for 0.5 seconds and posture changes in between the phases occur during a 0.5 second time frame.

The following two subsections present the results of performing the manoeuvre in Figure 3.10, first in the MSC.ADAMS software (Section 3.7.1.1.) and then in LMS.Virtual.Lab dynamics model (Section 3.7.1.2.). 


\subsubsection{MSC.ADAMS Simulation Results}

Prior to the availability of the high-fidelity LMS model, the basis of the proposed controller was tested on a simplified simulation model created in MSC.ADAMS. Due to the lack of information with regards to the hydraulic actuators of the actual robot, the input to the ADAMS model were defined as joint rates, the output of the inverse kinematics controller defined by Equation (3.30). Testing the controller in this manner allowed verification that the correct joint rates were implemented to attain the desired posture and trajectory inputs. The secondary benefit from implementation on ADAMS is also the ability to measure the moments applied by the hip and knee actuators. These moments are not directly available from the LMS model, and we are not able to compute them becausee of the closed nature of the actuator's model in LMS.

The resulting simulation tracked the desired signals with little or no error as seen in Figures $3.11,3.12$ and 3.13. These results indicate that the joint rates provided by the controller are accurate, without considering actuator dynamics.

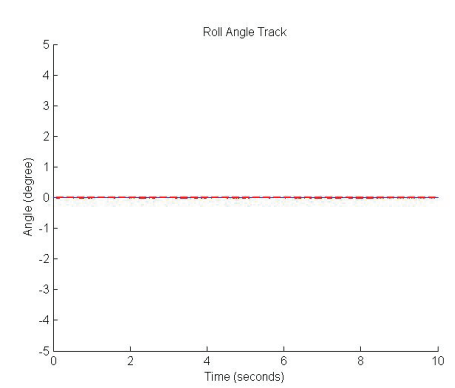

Figure 3.11: Roll tracking

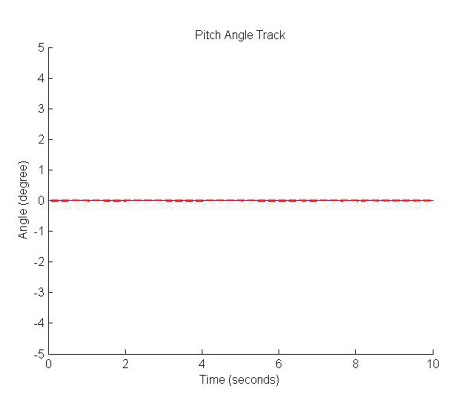

Figure 3.12: Pitch tracking

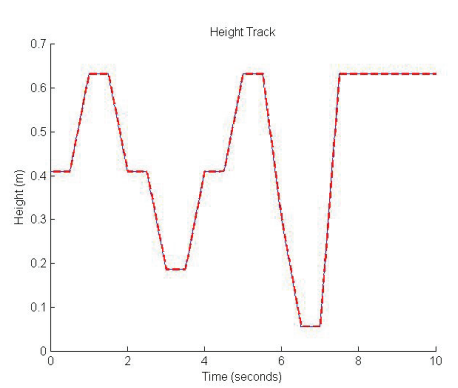

Figure 3.13: Height tracking

Using the simulation results from ADAMS, we can now inspect the moment arms occurring at each hydraulic actuator, as shown in Figures 3.14 and 3.15. As can be seen in the aforementioned figures, there are erratic changes in the torque measurements at 
every discrete change in the inputs. As this controller uses high gains in the inverse kinematics model, the erratic changes in torque are likely caused by the acceleration of the joints when the desired posture changes. This data only serves to give a general idea of the moments occurring at the hip and knee joints, as actuator dynamics are not considered. From this preliminary data, it can be easily seen that the highest torque occurs when MHT's platform is at its lowest, representing the most aggressive part of the maneuver. In addition, the torque in Figure 3.15 remains nearly zero before 5.5 seconds (up to Phase 6) and after 7.5 seconds (Phase 9) because the knee is located almost directly above the contact point, so that the vertical contact force causes little or no moment arm on the knee actuator.

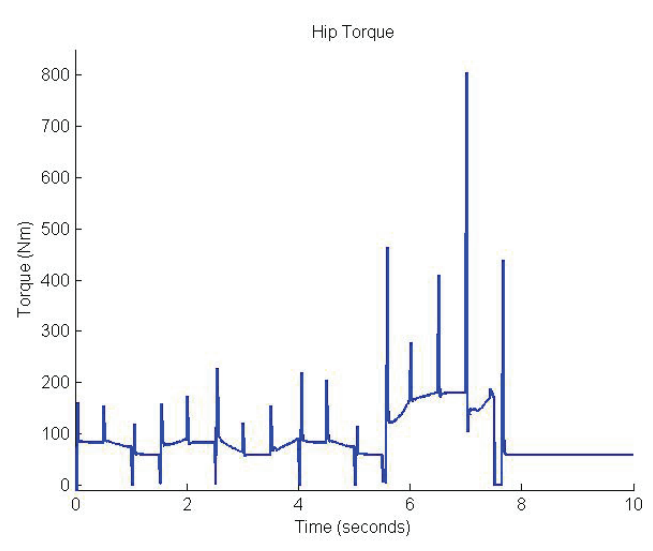

Figure 3.14: Front hip torque

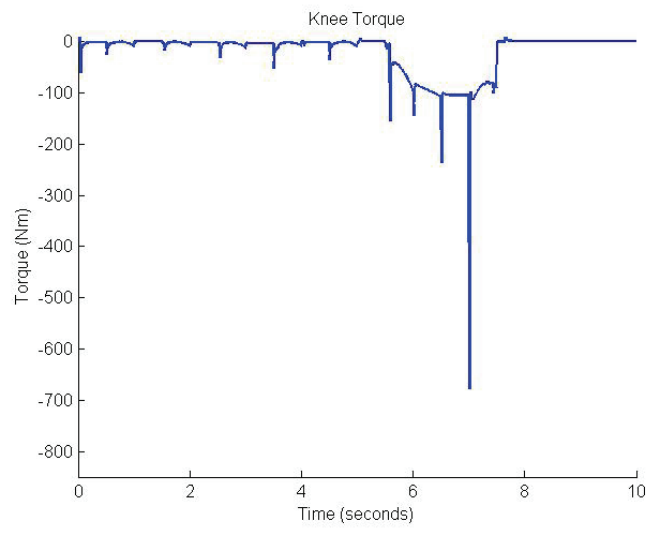

Figure 3.15: Front knee torque

Having confirmed the effectiveness of the inverse kinematics controller in MSC.ADAMS, the next step was implementation on the LMS model where actuator dynamics could be addressed.

\subsubsection{LMS Simulation Results}

The input tracking controller developed in this chapter resulted in accurate results when used with the LMS model, where MHT accomplished its task with a fast response time 
and no major runaway issues. Figures 3.16, 3.17 and 3.18 show the roll, pitch and height tracking behaviour between actual and desired values, respectively. Figure 3.19 shows all four wheel locations along the $x$-axis of the platform body-fixed frame.

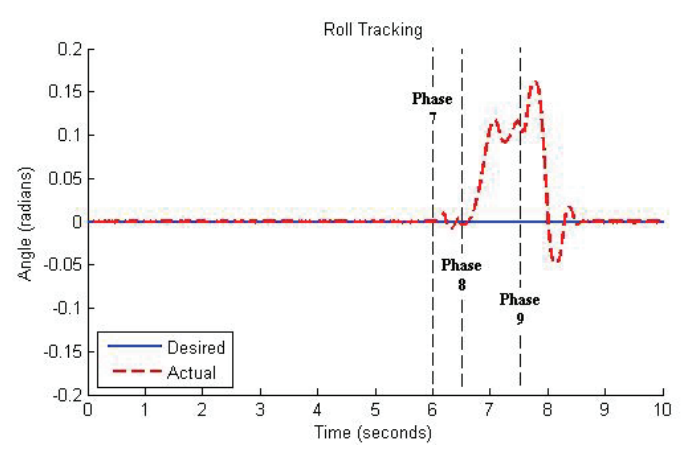

Figure 3.16: Roll tracking

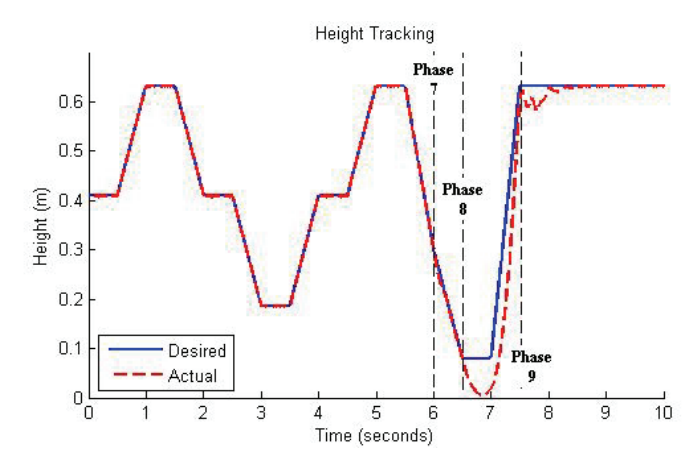

Figure 3.18: Height tracking

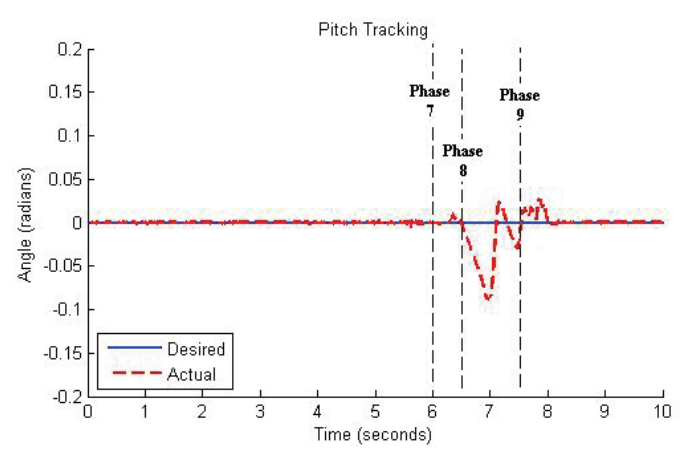

Figure 3.17: Pitch tracking

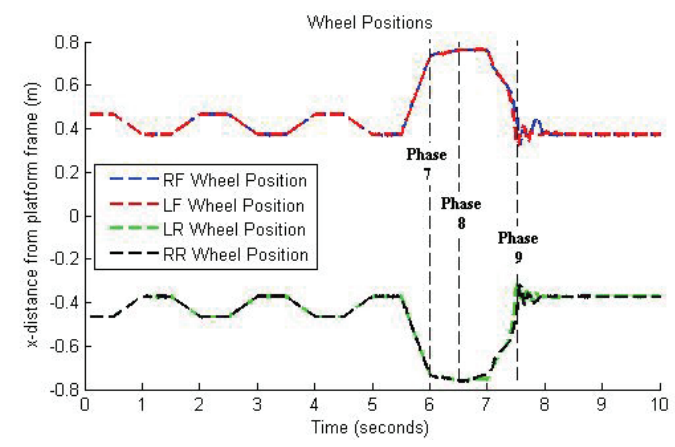

Figure 3.19: Wheel positions

The results from prescribing these posture inputs lead to very little tracking error and a fast response. However, the largest error occurred where MHT's center of mass was at its lowest point, resulting in a maximum error of $7.55 \mathrm{~cm}$ from the desired height. This is the most difficult portion of the manoeuvre as the legs are spread out, which causes a larger moment on the hips. In the end however, MHT regains its desired posture, where the error again becomes minor, without much overshoot. 


\subsubsection{Uneven Terrain Test Case}

The second test case challenges MHT to use its reconfigurable posture to adapt to an uneven terrain, which is not symmetric about the platform's sagittal plane. It has been shown that wheeled locomotion results in more energy efficient locomotion [50] than legged or stepping behaviours and although flat road surfaces are ideal for wheeled traversal, uneven terrain traversal must also be investigated to ascertain robustness of the controller. A topographical terrain layout used by [37] was created in LMS as shown in Figure 3.20, where (a) represents the terrain MHT's left side will traverse and (b) represents the terrain MHT's right side will traverse. The distances shown are measured with respect to the origin of the body-fixed frame when MHT is in its initial neutral position.

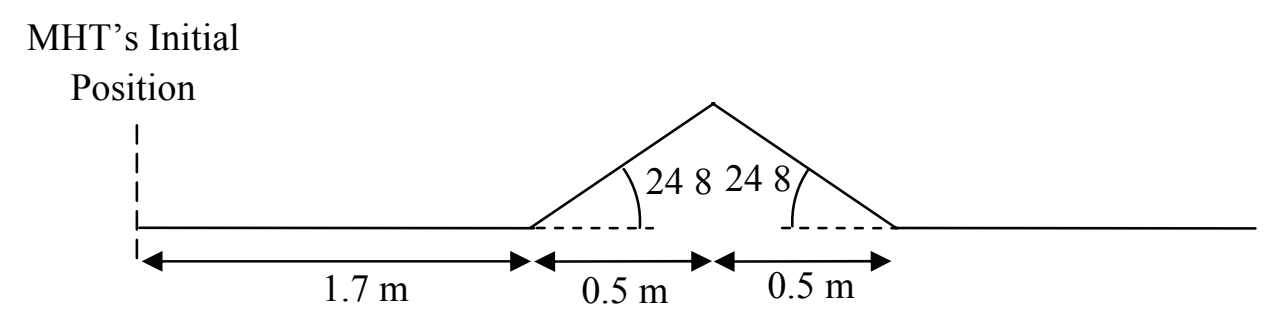

(a)

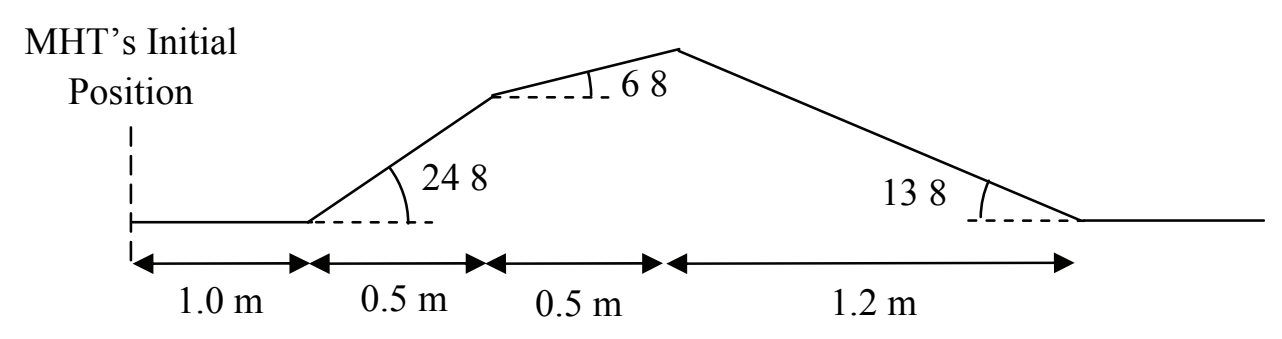

(b)

Figure 3.20: Uneven terrain height profiles (a) Left side (b) Right side

Furthermore, the snapshots shown in Figure 3.21 display the motion introduced, as simulated in LMS. 


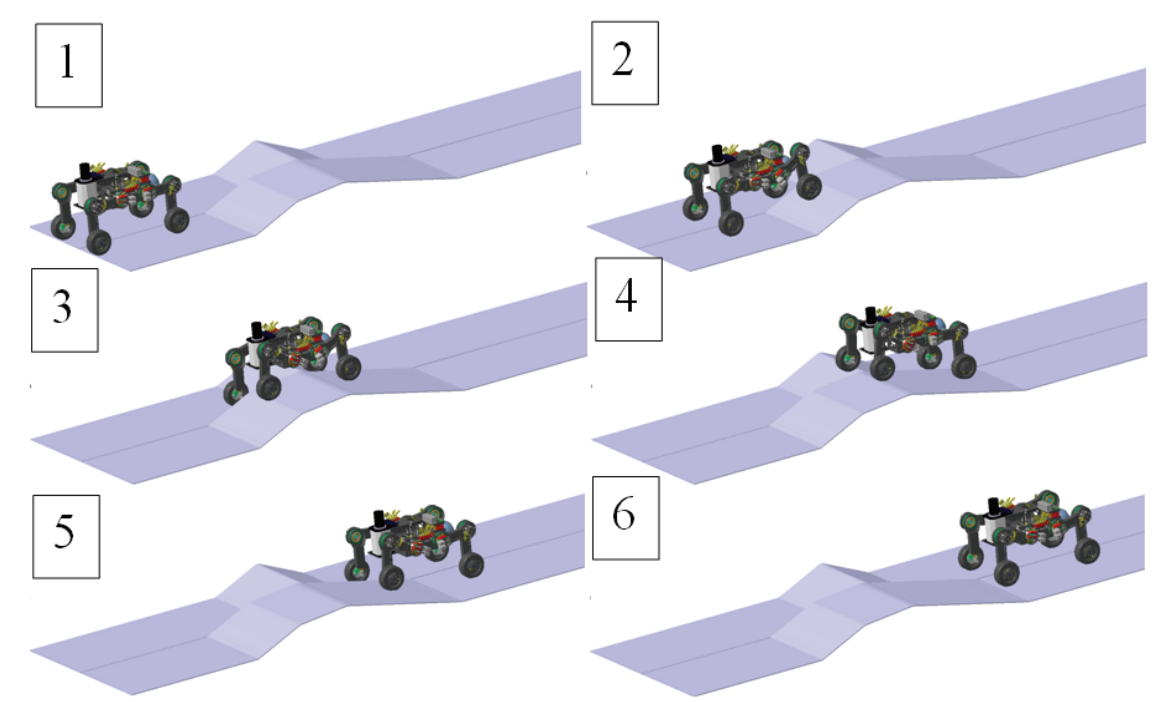

Figure 3.21: Uneven terrain motion snapshots

During this manoeuvre, the roll and pitch are maintained at zero degrees, while the height is prescribed at a value of $0.350 \mathrm{~m}$, the center of mass' initial elevation. The other input values are the $x$-position of each wheel, which are set at values of $\pm 0.465 \mathrm{~m}$, also corresponding to their initial distances and remain constant throughout the manoeuvre.

The desired and actual roll, pitch and height values can be seen in Figures 3.22, 3.23 and 3.24, respectively. As can be seen in these figures, the uneven terrain was traversed without much error. This demonstrated MHT's capability to overcome non-flat ground while maintaining the desired platform twist.

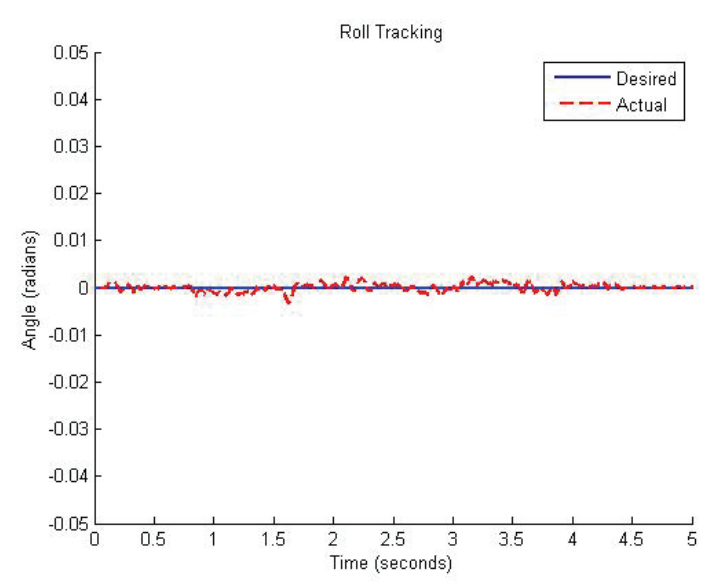

Figure 3.22: Roll tracking

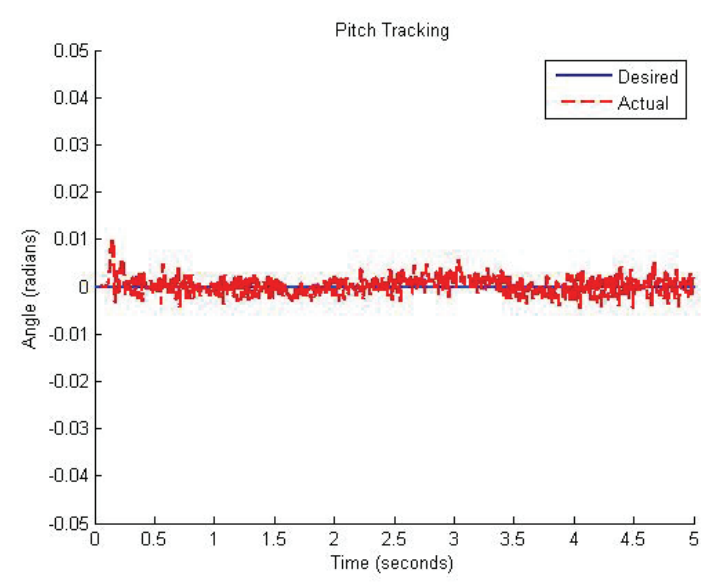

Figure 3.23: Pitch tracking 


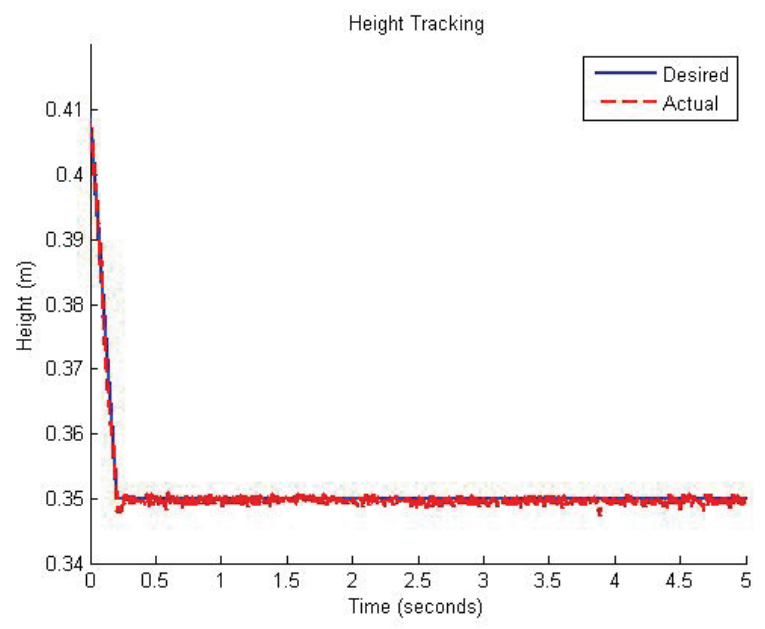

Figure 3.24: Height tracking

The preceeding figures show that little deviation from the desired values occur, however in the case of pitch, the actual value fluctuates slightly more, most likely due to the forward motion of the robot, although the pitch error still remains within $\mathrm{a} \pm 0.6^{\circ}$ margin . This could likely be corrected through gain tuning or applying a filter to smooth the signal.

\subsubsection{Independent Leg Control Test Case}

The independent leg control test case displays MHT's capability to define the motion of each articulated leg separately. This, in turn, allows MHT to be able to walk over obstacles possibly blocking its path. For this reason, it is essential to maintain sufficient control over the position of each wheel relative to the platform body. Tasks which require leg lift off are further complicated by the fact that stability becomes a concern when MHT is supported on three of its legs. Thus the primary objective of MHT under such a task is to first configure its posture into a stable position, where the projection of the center of gravity of the platform is located within the support polygon outlined by the 
three supporting legs, shown in Figure 3.25. In this posture, MHT can be commanded to raise its non-supporting leg to the necessary height.

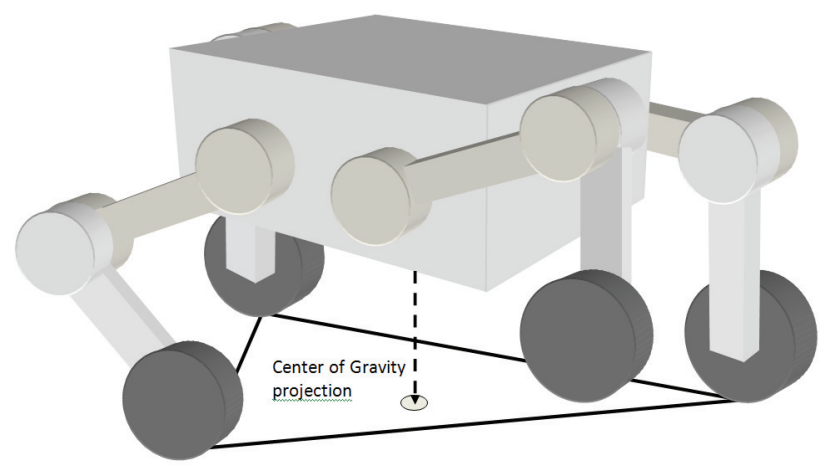

Figure 3.25: Three leg support polygon

For this particular case, the obstacle is a $0.254 \mathrm{~m}$ high step terrain feature, equal to the diameter of the wheel, and the posture and trajectory are defined with the knowledge of the location of the step. The full manoeuvre is shown by the snapshots in Figure 3.27. The prescribed trajectory input for motion in the $x$-direction is defined in Figure 3.26, where positive and negative values drive MHT forward and backward, respectively. The trajectory inputs are interpreted using an open loop control strategy, where a value of one signifies the maximum wheel actuator voltage to drive MHT forward and vice versa for negative one.

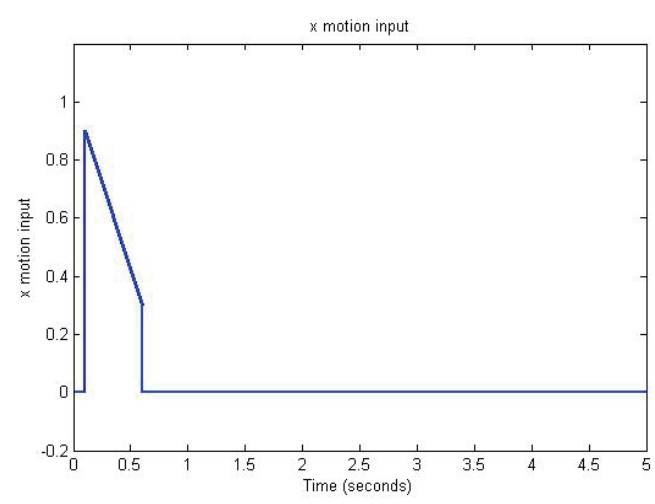

Figure 3.26: Trajectory input 
Based on Figure 3.26, MHT is commanded to drive forward and then to steadily slow down till the step feature is reached.

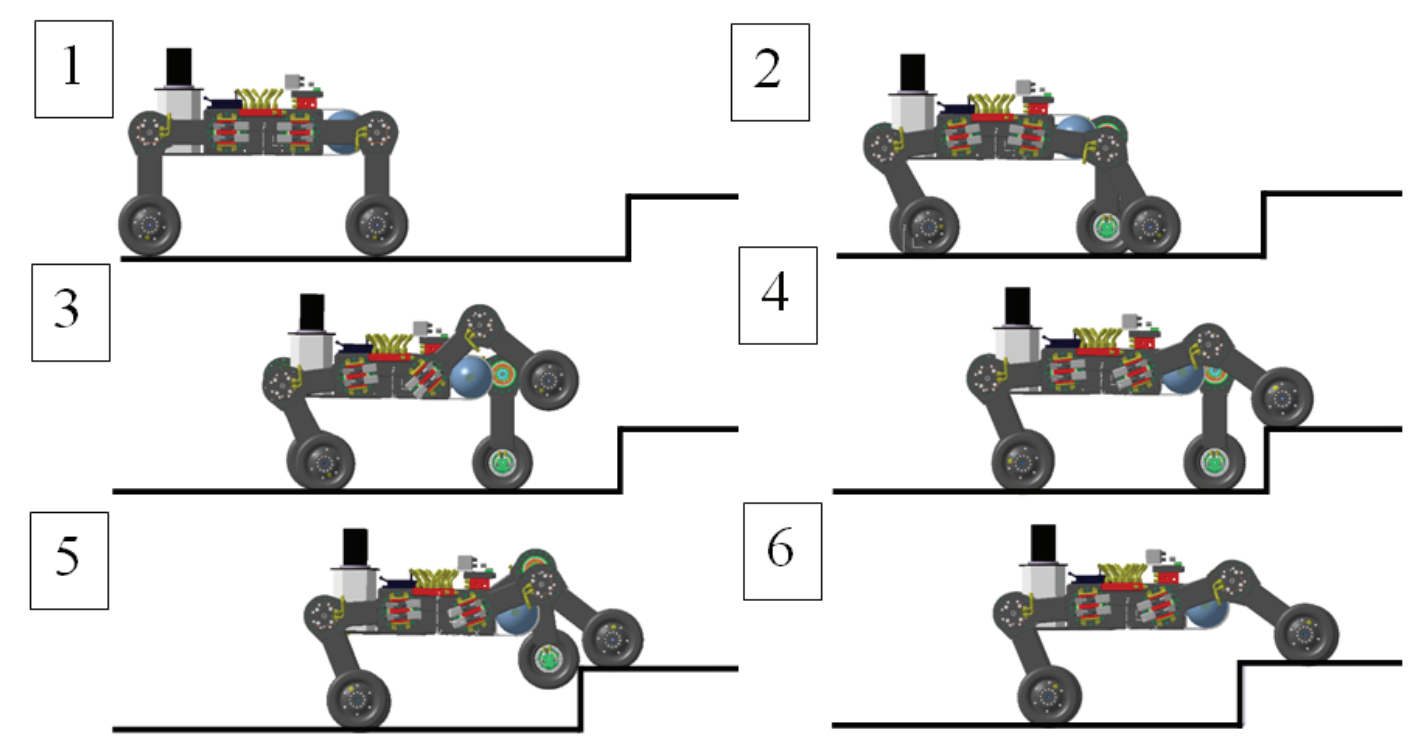

Figure 3.27: Leg lift manoeuvre snapshots

When performing this step manoeuvre, it is important to note that the input values were defined with the knowledge of the terrain prior to motion, unlike the case in the previous sections. This manoeuvre is conducted with only a $0.254 \mathrm{~m}$ step, not to show, not for the purpose of showing off how high a step MHT can negotiate, but to display the modified controller's capability to handle step features once exteroceptive sensors become available. The desired and actual posture inputs are shown in Figures 3.28 - 3.37.

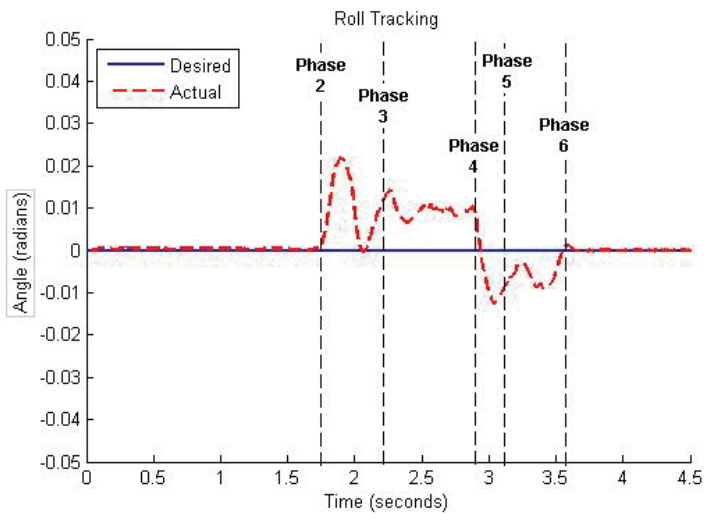

Figure 3.28: Roll tracking

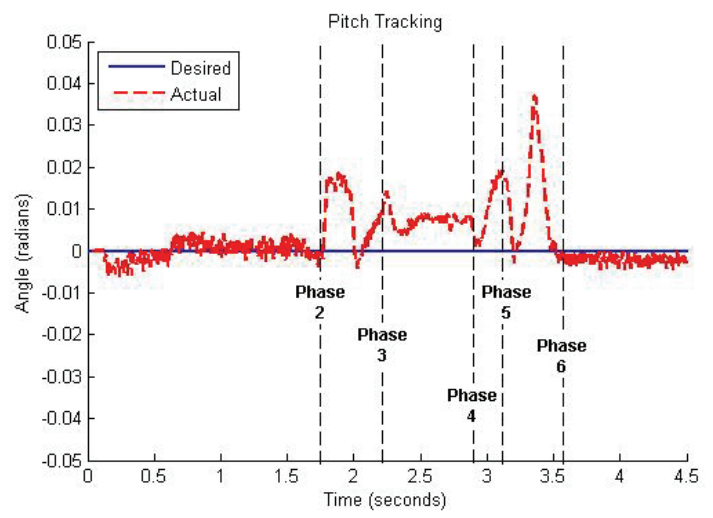

Figure 3.29: Pitch tracking 


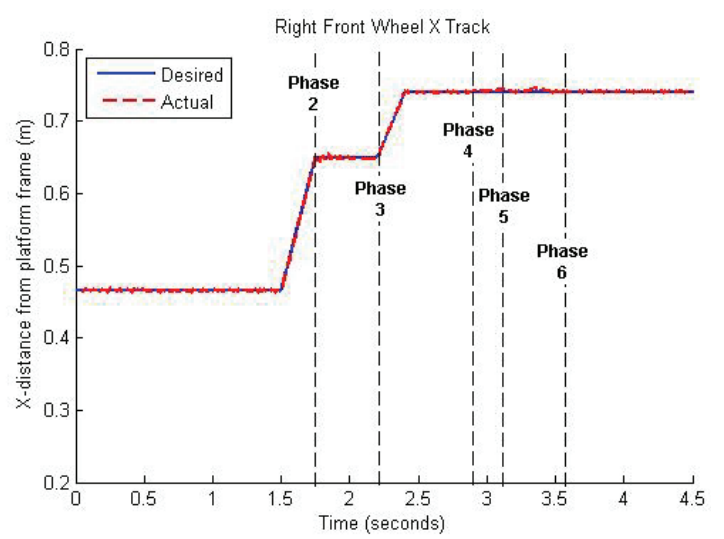

Figure 3.30: RF wheel $x$ tracking

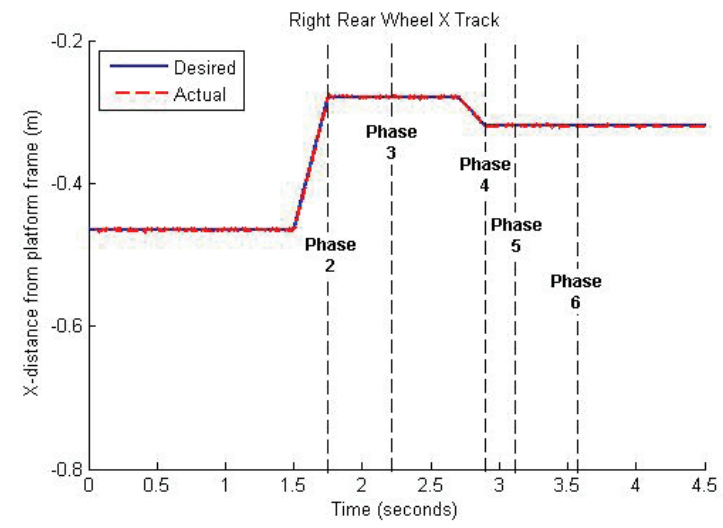

Figure 3.32: RR wheel $x$ tracking

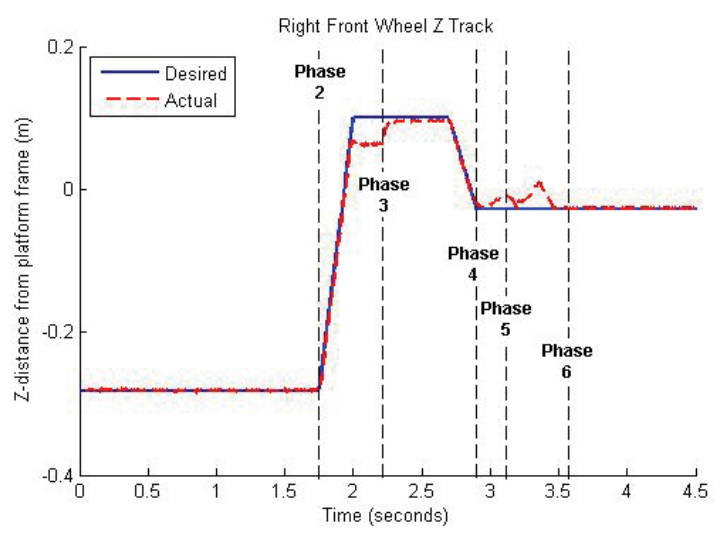

Figure 3.34: RF wheel $z$ tracking

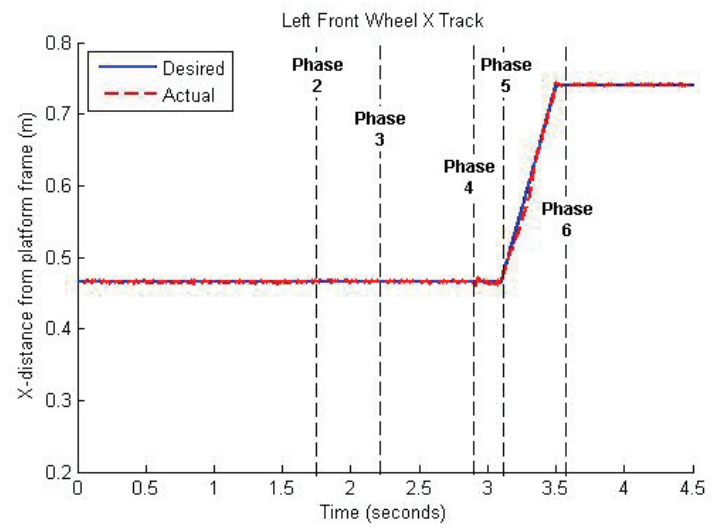

Figure 3.31: LF wheel $x$ tracking

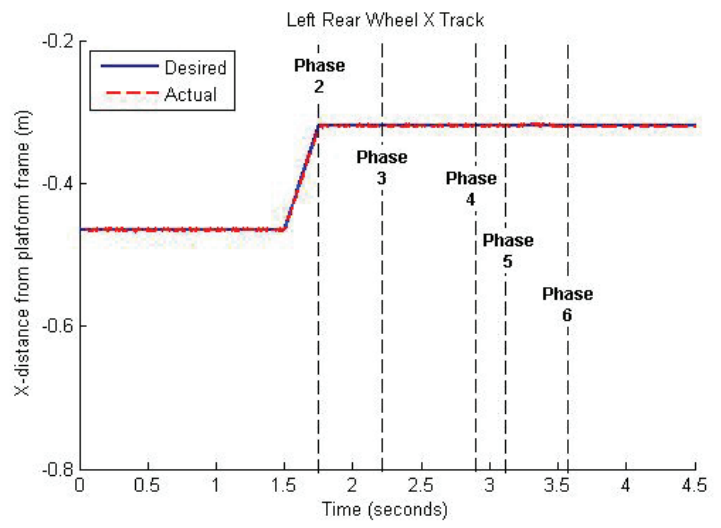

Figure 3.33: LR wheel $x$ tracking

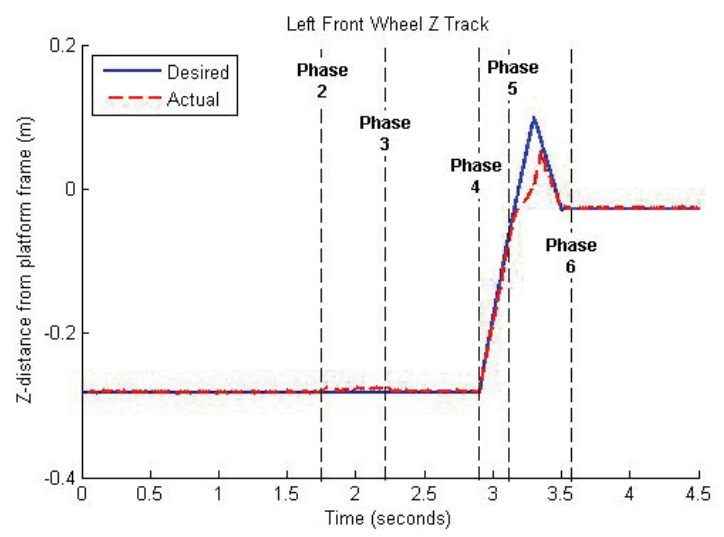

Figure 3.35: LF wheel $z$ tracking 


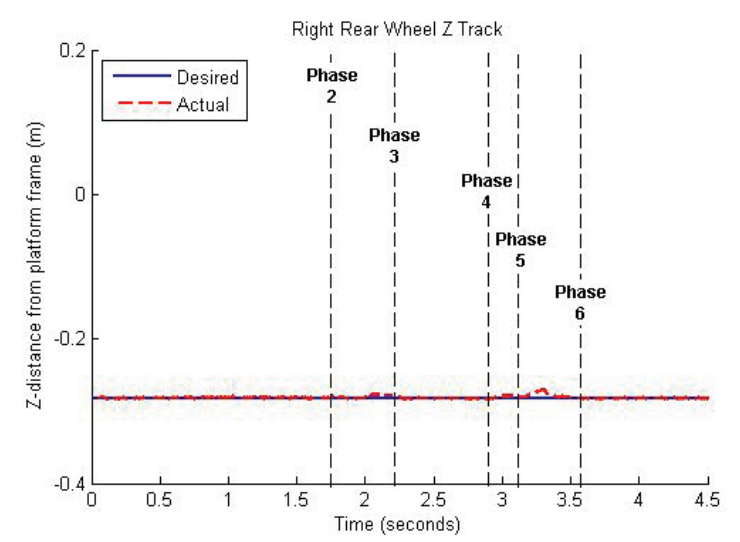

Figure 3.36: RR wheel $z$ tracking

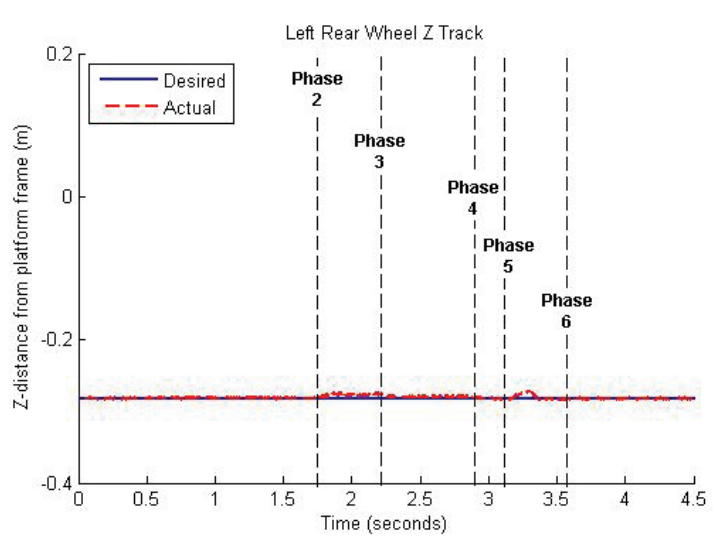

Figure 3.37: LR wheel $z$ tracking

For this case, the height value is omitted from the prescribed inputs, the reason being that the $z$-positions of each wheel relative to the platform are already defined, thus directly parameterizing the height of the body. The actual roll and pitch values, though small, tend to deviate somewhat erratically from their desired values, especially in comparison with the other manoeuvres shown in this chapter. This deviation is caused by the combination of the reduced stability when only on three legs (Figure 3.25) and the dynamic effects of reducing the forward velocity. Another issue that might play a role in this increased error is that the controller is solving an overdetermined system, which has no exact solution in general. Thus, some error is to be expected.

\subsection{Summary}

As shown in this chapter, using only the kinematics of the system, it is possible to effectively control the posture of a redundantly actuated hybrid quadruped robot. This is only a glimpse into MHT's potential, as trajectory feedback information will dramatically improve MHT's autonomous behaviour capability. The results produced with the three test cases demonstrate the good tracking behaviour between the actual and desired values. 
Though effective under the stated assumptions, the limitations of this control algorithm are the required knowledge of the terrain that cannot be driven over solely with wheels. 


\section{Chapter 4: Optimization}

\subsection{Introduction}

In the previous section, it was shown how a set of desired inputs defining the trajectory and posture are generated to achieve accurate posture control of MHT. However, it is important to describe criteria for the optimal posture that MHT will undertake, whether stationary or while in motion. When considering this problem, an important point to address, considering MHT's size and weight, are the moments acting on the joints. Therefore, the optimization procedure described in this chapter calculates the ideal posture to minimize the hip and knee actuator moments, which in turn alleviates the stresses on the platform due to gravity. One advantage to minimizing such torques is that this will contribute to a higher payload capacity for the platform. As MHT's envisioned functions are military in nature, an increase in payload can aid in carrying equipment or even incorporating more technology to aid MHT's tasks.

In formulating the optimization problem, the first assumption is that the motion is quasistatic with negligible acceleration, where frictional forces are considered to have minimal effect. Though this assumption will cause some error on sloped terrain, the calculation of torques will not be as accurate; however, this will be addressed indirectly in the objective function. In addition, we assume that the terrain is unknown prior to motion, but the wheels remain in contact with the terrain at all times. For the scope of this thesis, 
relatively low-angle sloped terrain is considered (less than 25 degrees with respect to the global frame). Under these circumstances, the horizontal contact forces will have a smaller effect in comparison to the vertical forces occurring at the contacts. Using these assumptions, this chapter will describe the procedure implemented to optimize the posture for MHT while minimizing a prescribed objective function.

\subsection{Design Variables}

The design variables are the robot parameters used to define both the objective function and optimization constraints. These variables are the basis for the optimal posture solution. The design variables chosen for this procedure are the static moments on each hip and knee actuator, $\tau_{H i p, i}$ and $\tau_{\text {Knee, } i}$ respectively, caused by MHT's weight; these will contribute eight design variables. Another variable incorporated in the objective function, is the platform height, $z$, relative to the four wheels, which is minimized to a specified value to maintain a certain amount of ground clearance to avoid platform contact with the terrain.

\subsection{Constrained Nonlinear Optimization}

The selected optimization technique is the nonlinear programming method [51], which determines a locally minimizing solution for a selected objective function [51]. As shown by Nahon [49], there are several potential strategies for creating an effective objective function. The particular optimization technique used allows both linear and non-linear constraints, both through equality and inequality equations. 
The optimization process, for MHT's case, has 19 inputs, which parameterize the necessary constraints to characterize the robot's kinematic configuration. Nine of these variables are used in the optimization objective function and are called design variables, as mentioned in Section 4.2. The remaining 10 optimization inputs consist of the current angular position of each hip and knee actuators, $\alpha_{i}$ and $\beta_{i}$ respectively, and the roll, $\varphi$, and pitch, $\psi$, of the body platform.

The optimization method functions using an initial guess obtained from the current kinematic state of MHT defined by the 19 variables previously mentioned. The goal of the optimization process is achieved by minimizing the objective function described in Section 4.5. To properly frame the necessary constraints for the optimization, Eqs. (4.1) and (4.2) show the form of the linear constraints, which will be explained in greater detail in Section 4.4.

$$
\begin{gathered}
\mathbf{A}_{\text {ineq }} \mathbf{x} \leq \mathbf{b}_{\text {ineq }} \\
\mathbf{A}_{e q} \mathbf{x}=\mathbf{b}_{e q}
\end{gathered}
$$

The matrix $\mathbf{A}_{\text {ineq }}$ and vector $\mathbf{b}_{\text {ineq }}$ represent the linear inequality constraints, while the matrix $\mathbf{A}_{e q}$ and vector $\mathbf{b}_{e q}$ define the linear equality constraints and $\mathbf{x}$ is the optimization solution. Furthermore, lower and upper bounds are defined to represent MHT's kinematic limits. Finally, a set of constraints describing the nonlinear kinematics and simplified dynamics of MHT are shown in Section 4.4.

When formulating the optimization strategy, it is important to note that both the initial guess and optimized solution must satisfy all the defined constraints for the optimization 
to operate properly. If these conditions are not met, the operation will result in a "no solution" result. For this reason, the constraints cannot be defined too strictly or the optimization may fail.

The optimization solution is obtained using MATLAB's 'fmincon' function. The most notable issue to consider when applying 'fmincon' is that the solution found represents only the local minimum and is not guaranteed to be a global optimum. This means that the optimizer does not necessarily find the best possible solution, but rather only a better solution from the initial guess supplied. Thus, the initial guess plays a vital role in determining the solution obtained by the optimizer.

\subsection{Constraint Details}

The constraints defined to calculate an optimum, are kinematic in nature and based on quasi-static motion. Considering MHT is a non-linear system, few linear constraints are necessary for the optimization procedure. First, the system of linear inequalities $\mathbf{A}_{\text {ineq }}$ and $\mathbf{b}_{\text {ineq }}$ are prescribed as empty, while the only linear equalities applied are to maintain a level body with respect to ground, where roll and pitch are constrained to zero:

$$
\begin{aligned}
& \varphi=0^{\circ} \\
& \psi=0^{\circ}
\end{aligned}
$$

The following set of constraints describe the upper and lower bounds, which confine the optimization solution to within the hydraulic actuator limits. The upper and lower bounds of the optimization are prescribed as follows: 


$$
\begin{gathered}
-45^{\circ} \leq \alpha_{i} \leq 45^{\circ} \quad, i=1,2,3,4 \\
-45^{\circ} \leq \beta_{i} \leq 45^{\circ} \quad, i=1,2,3,4 \\
0.08 \mathrm{~m} \leq z \leq 0.632 \mathrm{~m}
\end{gathered}
$$

The bounds for each of the hip and knee angles correspond to the physical limits of the actuators, which have a full range of motion of $90^{\circ}$, as described in Chapter 2 . The limits for the height parameter are shown in Figure 4.1 and 4.2, assuming flat terrain. The lower bound for height is defined empirically and is the lowest desirable average height under a stable stationary configuration on flat terrain. The highest possible height for MHT is the posture in which all the legs are extended to their maximum vertical length on level terrain.

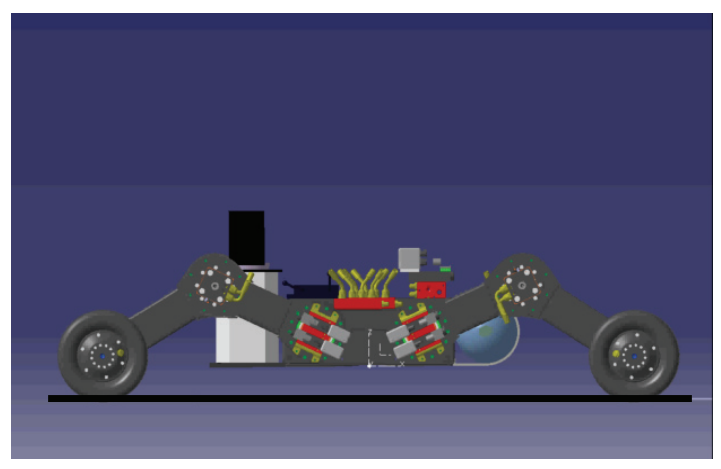

Figure 4.1: Minimum allowable height

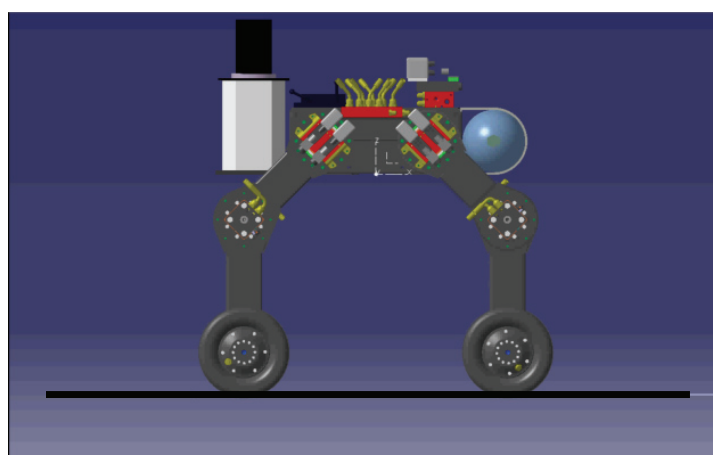

Figure 4.2: Maximum allowable height

The following equations define the nonlinear constraints that the optimization procedure must satisfy and effectively embody the static equilibrium of the platform.

$$
\begin{gathered}
\tau_{\text {Кпее }, i}=F_{y_{i}} \cdot b_{x_{i}}-l_{k t, i} \cdot m_{t} \cdot g-l_{k w, i} \cdot m_{w} \cdot g \quad, i=1,2,3,4 \\
\tau_{\text {Hip }, i}=F_{y_{i}} \cdot a_{x_{i}}-l_{h f, i} \cdot m_{f} \cdot g-l_{h t, i} \cdot m_{t} \cdot g-l_{h w, i} \cdot m_{w} \cdot g \quad, i=1,2,3,4
\end{gathered}
$$

The torques of the $i^{\text {th }}$ leg hip and knee are, $\tau_{H i p, i}$ and $\tau_{K n e e, i}$, respectively, $F_{y i}$ is the vertical contact force of the $i^{\text {th }}$ wheel and $g$ is the gravitational constant. The values $a_{x i}$ and $b_{x i}$ are 
parameterized by the joint angles of $i^{\text {th }}$ leg and are shown in Figure 4.3. The variables $m_{f}$, $m_{t}$ and $m_{w}$ represent the mass of the femur, tibia and wheel, respectively. Finally, values $l_{k t, i}$ and $l_{k w, i}$ are the distances from the knee joint to the center of mass of the tibia and wheel, while $l_{h f, i}, l_{h t, i}$ and $l_{h w, i}$ are the distances from the hip joint to the center of mass of the femur, tibia and wheel, respectively. The vertical contact force is assumed constant and equal to a quarter of the overall weight of MHT:

$$
F_{y_{i}}=\frac{m g}{4}=357.82 \mathrm{~N} \quad, i=1,2,3,4
$$

The variable $m$ represents the total mass of MHT, as defined in Table 2.1, and $g$ is the gravitational constant. The actuator moments are displayed in Figure 4.3, using the right hand rule for positive or negative torque.

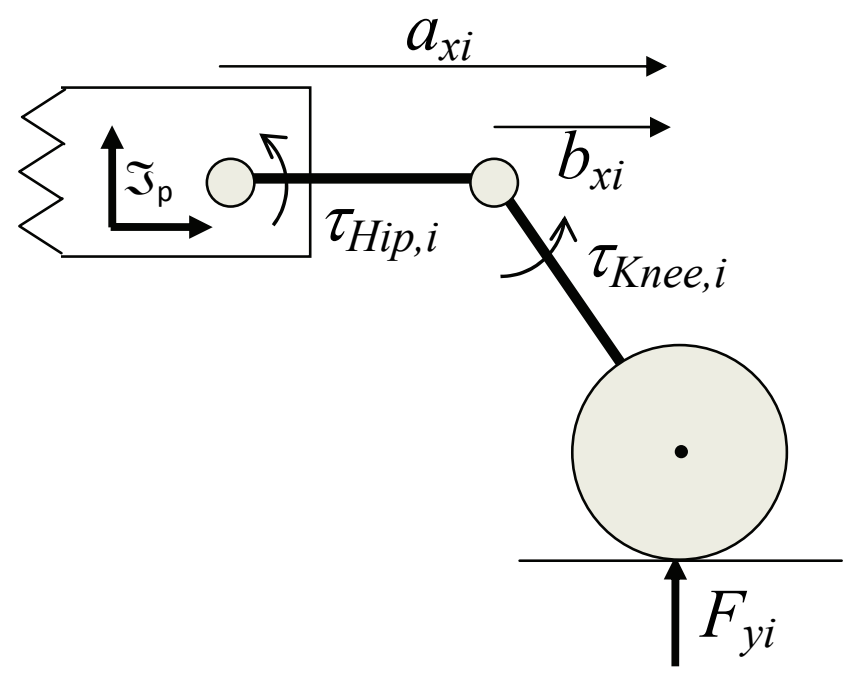

Figure 4.3: Joint torque definition

It is important to note that the torques calculated by Equations (4.4) and (4.5) are very conservative. These equations consider the moment caused by the weight of each individual link as a point mass and simplifying assumptions are made for ground contact force distribution. 
The next set of constraints stated in Equations (4.6), (4.7) and (4.8) restrict the optimization solution to a local neighbourhood near the initial guess. By omitting these constraints, the optimized solution may be distant from the initial guess, causing an abrupt change to the inputs of the controller. This is not desirable as sudden changes to the controller inputs may cause erratic manipulator movement and overshoot of the desired value supplied by the optimization. Therefore, Eq. (4.6), (4.7) and (4.8) define nonlinear constraints that limit the optimized solution change from the initial guess (current kinematic configuration).

$$
\begin{gathered}
\left|z-z_{0}\right| \leq \varepsilon_{1} \\
\left|r_{x_{i}}-r_{0 x_{i}}\right| \leq \varepsilon_{2} \\
\left|r_{z_{i}}-r_{0 z_{i}}\right| \leq \varepsilon_{3}
\end{gathered}
$$

In the above, $r_{x i}, r_{z i}$ and $z$ represent the optimized solution and $r_{0 x i}, r_{0 z i}$ and $z_{0}$ denote the corresponding initial guess, where $r_{x i}$ and $r_{z i}$ are the $x$ - and $z$-component of the vector $\mathbf{r}$ defined in Figure 3.1. Finally, $\varepsilon_{1}, \varepsilon_{2}$ and $\varepsilon_{3}$ represent small, empirically defined, finite neighbourhoods around the initial guess to which the optimized solution is restricted. The optimization process presented here assures a more gradual change of the controller inputs, allowing for a smoother response of MHT, thus controlling the change between optimized solutions from the current kinematic configuration. This idea was proposed by Nahon [49], but he achieved minimal changes in applied actuator torques by accounting for these within the objective function, rather than with the constraints, as is done here. 
Furthermore, the optimization function produces a new set of posture inputs for the controller at every $0.01 \mathrm{~s}$ of simulation time. This optimization sampling time was chosen arbitrarily, though through trial and error, it was noticed that smaller sampling times $(<$ $0.01 \mathrm{~s})$ would increase the simulation run time. Secondly, large optimization sampling times $(>0.01 \mathrm{~s})$ would yield larger changes between optimized posture inputs, causing less continuous posture transition throughout the manoeuvre.

\subsection{Objective Function}

The objective function to be minimized is expressed in Equation (4.9):

$$
J=K_{1}\left(z-z_{\text {des }}\right)^{2}+K_{2}\left(\boldsymbol{\tau}_{\text {Hip }}^{\mathrm{T}} \boldsymbol{\tau}_{\text {Hip }}\right)+K_{3}\left(\boldsymbol{\tau}_{\text {Knee }}^{\mathrm{T}} \boldsymbol{\tau}_{\text {Knee }}\right)
$$

where $K_{1}, K_{2}$ and $K_{3}$ are the weights, $z$ is the optimized height value, $z_{\text {des }}$ is the goal height value, $\tau_{H i p}$ is the vector of hip torques and $\tau_{\text {Knee }}$ is the vector of knee torques. The result of minimizing this objective function is to move the robot height towards the desired value, while minimizing all the torques occurring at the actuators.

The gains selected to weigh the hip and knee torques were first chosen empirically and tuned through trial and error, to balance the torque distribution between the hips and knees.

\subsection{Simulation Results}

To test the result of applying the optimization procedure described in the previous sections to MHT, the same uneven terrain topology is used as the second simulation test case in chapter 3, Figure 3.20. As previously discussed, the goal of the objective function 
is to minimize the moments calculated by Eqs. (4.4) and (4.5) acting on each hip and knee actuator, while maintaining the desired height.

On flat terrain, MHT attains the posture shown in Figure 4.4. In this case, the desired height value, $z_{\mathrm{des}}$, for Equation (4.9) is chosen as $0.35 \mathrm{~m}$.

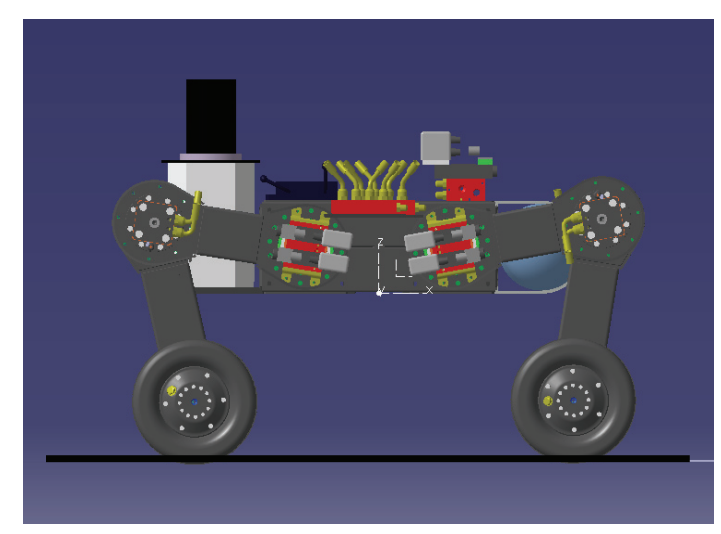

Figure 4.4: Optimized posture on flat terrain

As mentioned in Section 4.4, the moments calculated using Equations (4.4) and (4.5) will be conservative with respect to the actual moments. The calculated torques for hips and knees were approximately $59.5 \mathrm{Nm}$ and $21.5 \mathrm{Nm}$, respectively. While in MSC.ADAMS, the measured torque for the hip and knees of this posture was shown to be $57.0 \mathrm{Nm}$ and 23.7 Nm, respectively. Though conservative, these calculations show good accuracy with respect to their actual values.

The snapshots in Figure 4.5 show the optimization being applied on-line over the uneven terrain specified in chapter 3. As shown, when MHT encounters the sloped terrain at approximately 0.70 seconds, it maintains its body configuration and adjusts its legs configuration whereby the actuator moments are minimized. 


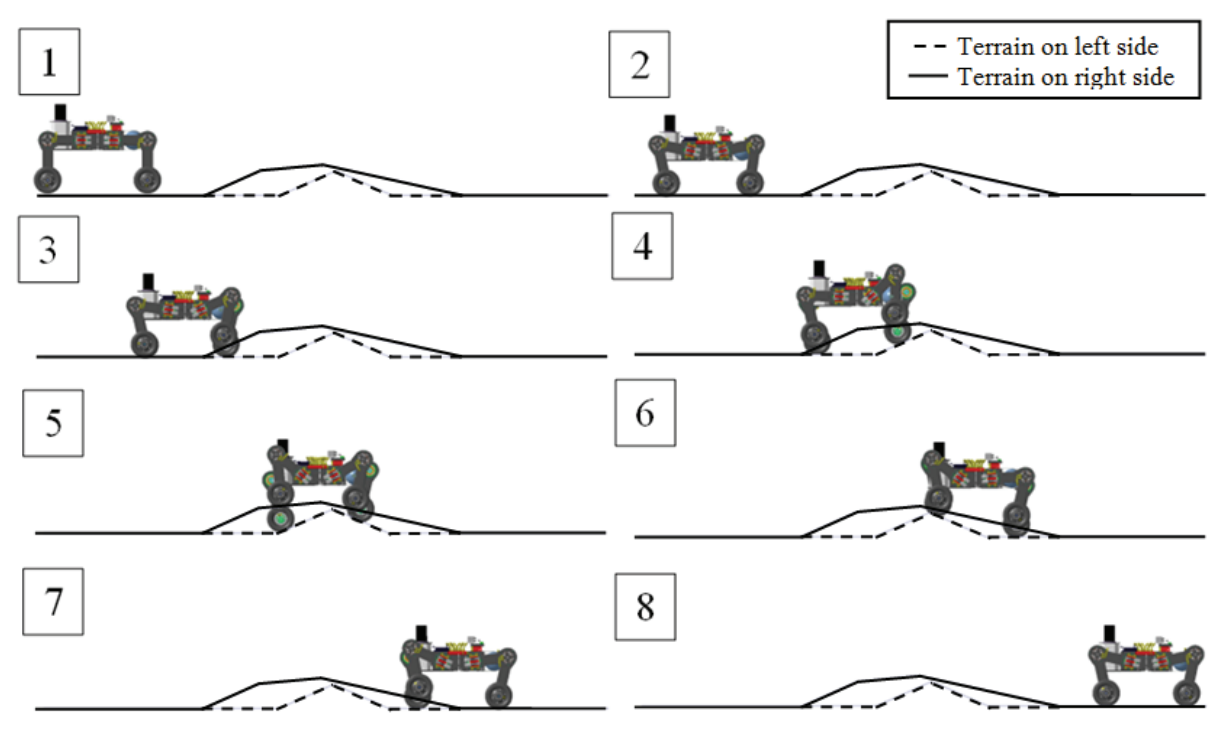

Figure 4.5: Optimized uneven terrain manoeuvre

As previously mentioned, since terrain topology is unknown prior to motion and cannot be detected through exteroceptive sensors, only the approximate vertical contact forces can be used in the optimization solutions as per Equations (4.4) and (4.5). Though this does not cause much of a discrepancy from the actual contact forces on flat terrain, sloped terrain changes the magnitude and direction of the normal force. When MHT's wheels travel on a non-level terrain, as shown in Figure 4.6, the normal forces vary and horizontal forces occur and increase with a larger slope. Since only shallow slopes are considered in this thesis, the contact point will be assumed to remain constant at the base of the wheel, to simplify the kinematic calculations within the inverse kinematics controller. Furthermore, as terrain topology is not explicitly known prior to locomotion, the horizontal forces cannot be directly accounted for in the minimization procedure. Unlike the torques due to vertical forces, which are based on $a_{x i}$ and $b_{x i}$, the horizontal ground force will apply a torque parameterized by $a_{z i}$ and $b_{z i}$ on the hip and knee actuators, respectively. Therefore, to indirectly minimize the moments caused by the horizontal force, the desired height, $z_{\text {des }}$, is prescribed within the objective function, as 
described in Section 4.5. Defining this height also maintains a satisfactory clearance over possible terrain obstructions.

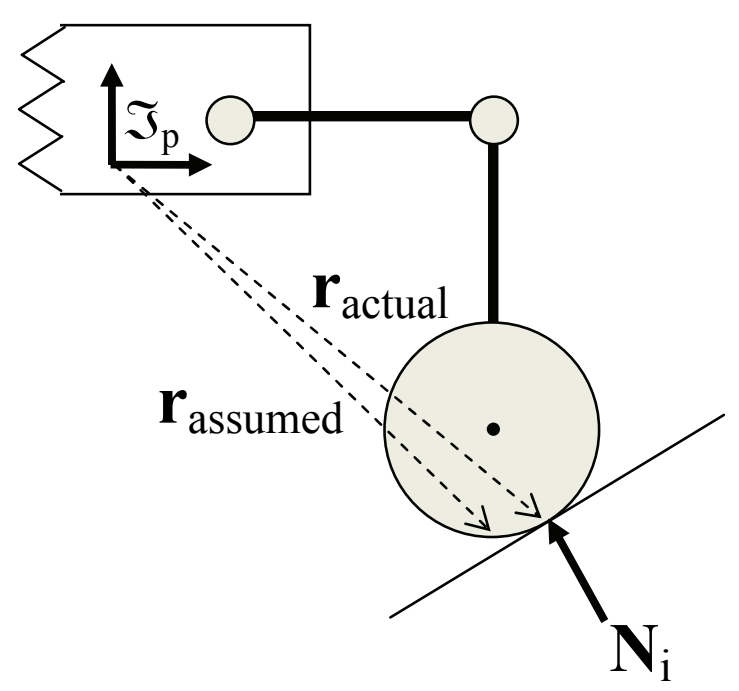

Figure 4.6: Force diagram of slope contact

The desired and actual roll, pitch and height values for this manoeuvre can be seen in Figures 4.7, 4.8 and 4.9, respectively. Throughout the entire motion, the roll and pitch are maintained at zero degrees and the individual legs accommodate the terrain, while maintaining wheel-ground contact. Figure 4.9 shows that on flat terrain, a height of 0.35 $m$ is maintained quite accurately, whereas during the routine over the uneven terrain, the height fluctuates by as much as $4 \mathrm{~cm}$ from the desired value. Once level terrain is encountered again, the height re-stabilizes at the defined $z_{\text {des }}$ value of $0.35 \mathrm{~m}$. The optimized height value fluctuates from $z_{d e s}$ due to the definition of the objective function, where the gains are chosen to favour of minimization of the torques as opposed to the deviation in height. This is desirable as it allows the legs to effectively adapt to the terrain while not enforcing too strict a constraint on the height value. As described by Equations (4.4) and (4.5), the moments are based on the $x$-distance between each actuator and each wheel. 


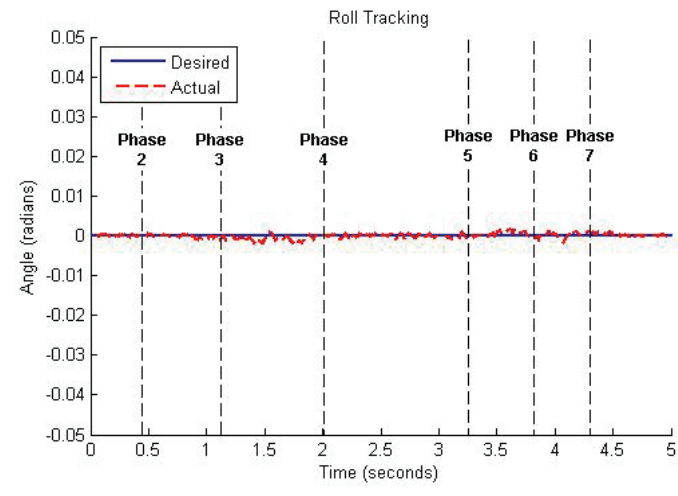

Figure 4.7: Roll tracking

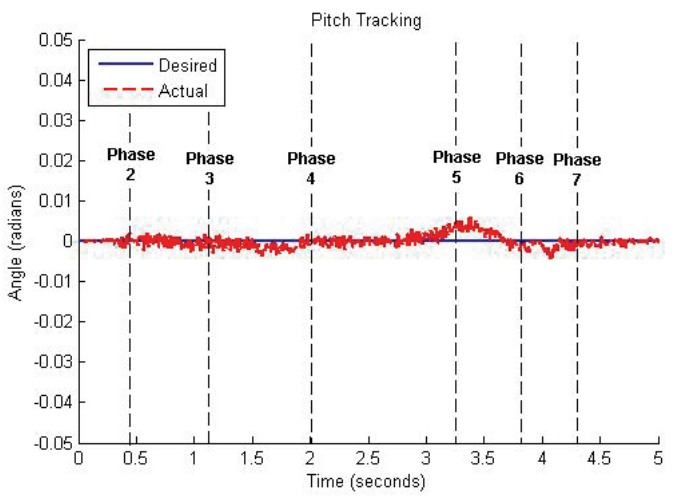

Figure 4.8: Pitch tracking

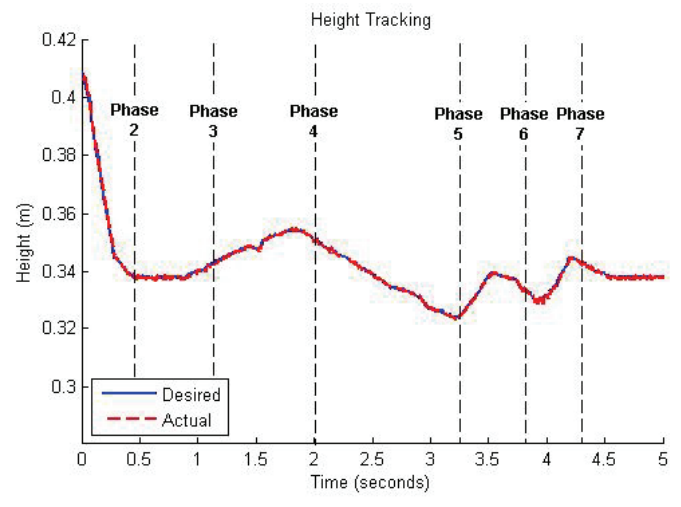

Figure 4.9: Height tracking

In Figures 4.10, 4.11, 4.12 and 4.13, it can be seen that the $x$-position of each wheel, with respect to the body frame origin, remains relatively constant.

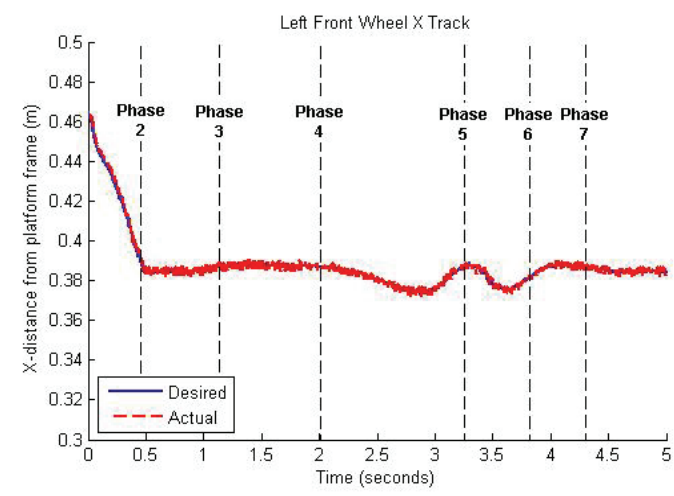

Figure 4.10: Left front wheel $x$-distance

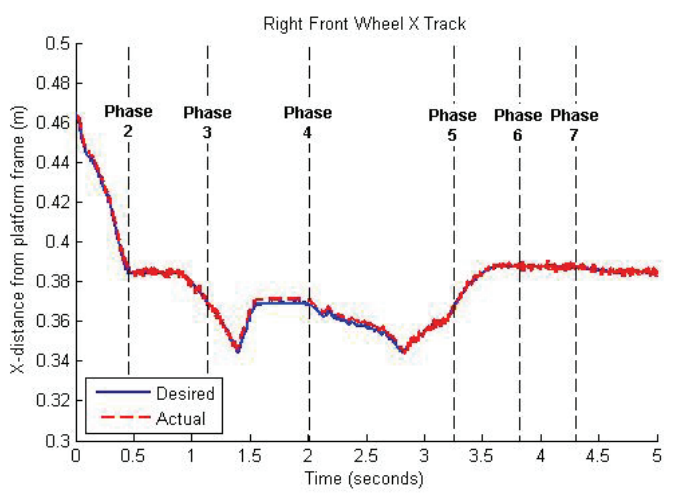

Figure 4.11: Right front wheel $x$-distance 


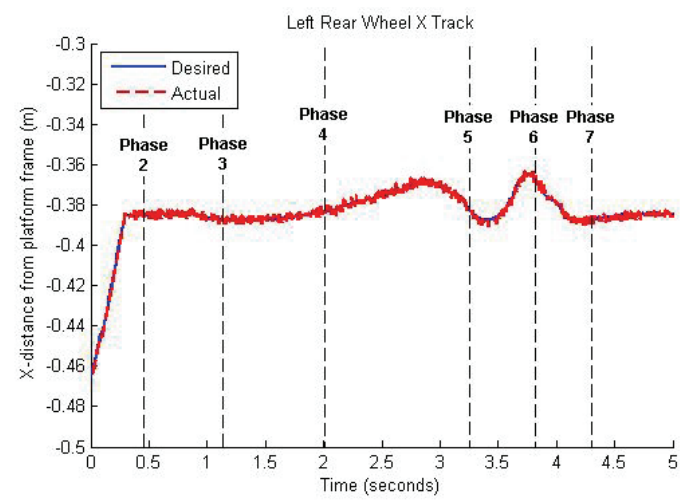

Figure 4.12: Left rear wheel $x$-distance

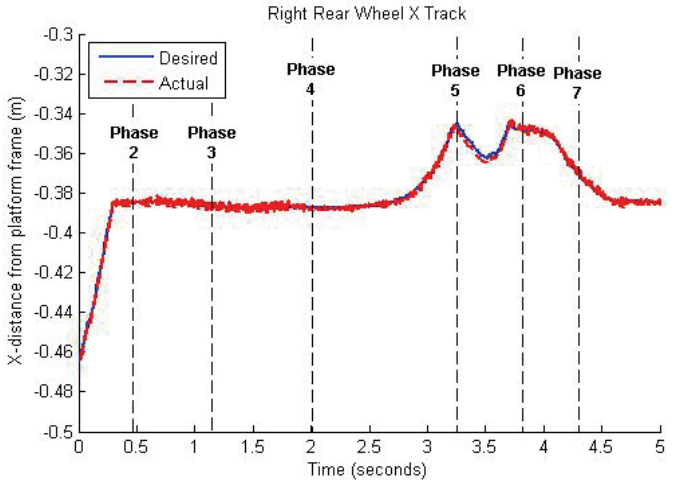

Figure 4.13: Right rear wheel $x$-distance

Though there are variations in the wheels' $x$-positions, these remain at less than $4 \mathrm{~cm}$ in magnitude; these changes can be attributed to the instances when a wheel makes contact with a change in slope. The fluctuation in each wheel position is due to the actual horizontal force occurring on the sloped terrain, which cannot be estimated. However, as the assumptions made for this optimization only account for the vertical contact forces, the optimized posture re-adjusts the positions of the wheels to minimize the torques defined by Equations (4.4) and (4.5).

Furthermore, the individual hip and knee actuator torque comparisons between the nonoptimized case (see Section 3.7.2.) and the optimized case, as calculated by Eqs. (4.4) and (4.5), are displayed in the Figures 4.14, 4.15, 4.16 and 4.17. The torque comparisons show a decrease in the hip torques and an increase in the knee torques, improving the torque distribution among the actuators. 


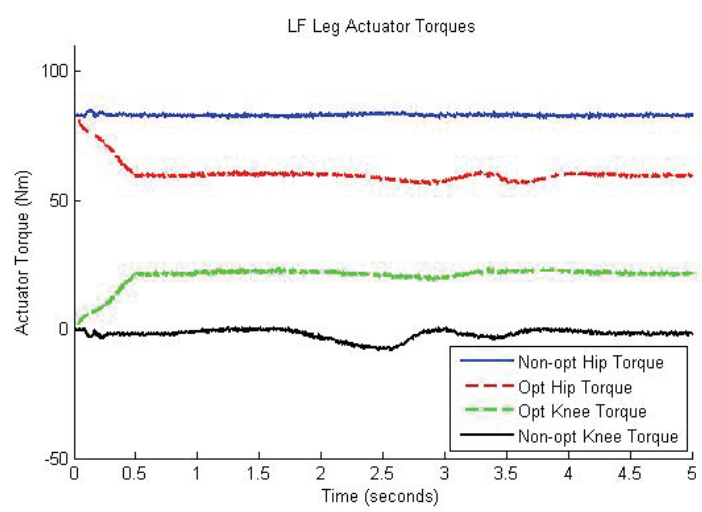

Figure 4.14: LF leg actuator torques

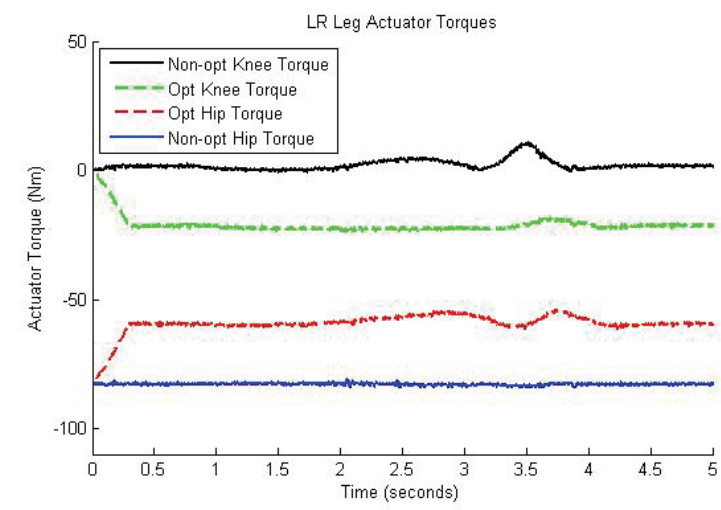

Figure 4.16: LR leg actuator torques

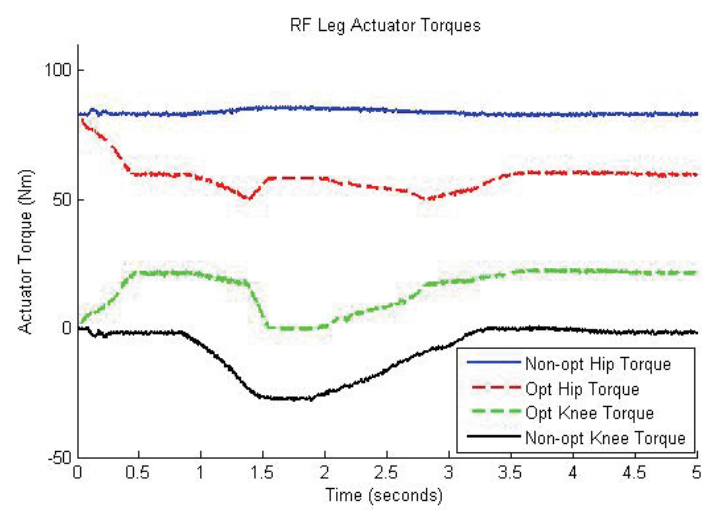

Figure 4.15: RF leg actuator torques

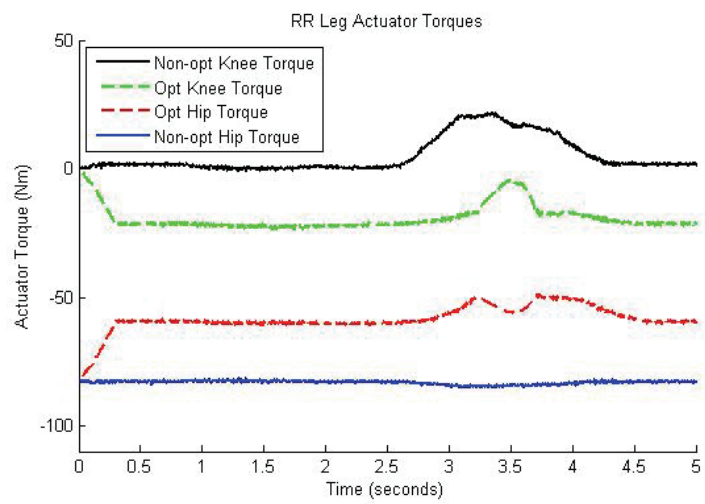

Figure 4.17: RR leg actuator torques

To better ascertain the performance of the posture optimization, the method of assessment selected is to compare the squared norm of the torques for both cases, i.e.:

$$
\|\tau\|^{2}=\left(\boldsymbol{\tau}_{\text {Hip }}^{\mathrm{T}} \boldsymbol{\tau}_{\text {Hip }}\right)+\left(\boldsymbol{\tau}_{\text {Kneе }}^{\mathrm{T}} \boldsymbol{\tau}_{\text {Knеe }}\right)
$$

As shown in Fig. 4.18, the squared norm of the torques is significantly reduced for the optimized solution when compared to the non-optimized result. This confirms the overall reduction on the torque load and, as a side effect; the optimized solution improves the torque distribution among the actuators. 


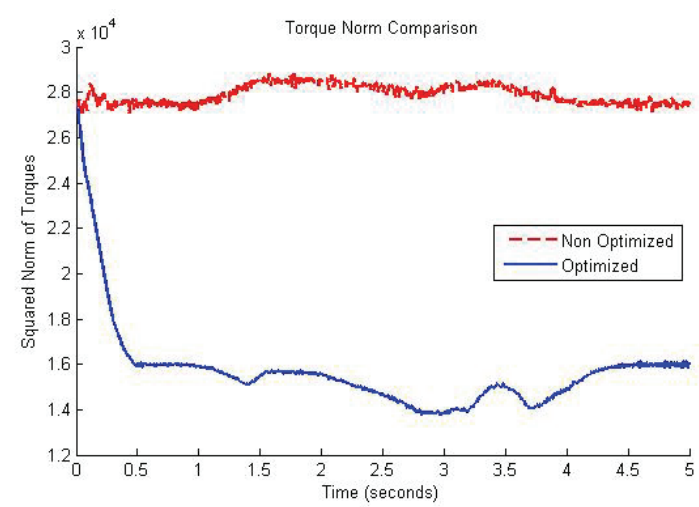

Fig. 4.18: Torque Norm comparison

\subsection{Summary}

This chapter has demonstrated the optimization of MHT's posture using the specified constraints. By forcing the optimized solution to lie in the neighbourhood of the initial guess, the formulation allows a smoother change between optimized posture inputs for the inverse kinematic controller. This provides several benefits, such as a more gradual adjustment in MHT's posture and an optimized solution to the initial guess. The result of the optimization effectively provides a posture whereby the loads caused by the robot's weight are more balanced between the hip and knee actuators, through quasi-static analysis. The proposed optimization solution can allow an increase in MHT's payload travelling over unknown terrain, while attempting to avoid overloading any of the hydraulic actuators. 


\section{Chapter 5: Conclusions and Future Work}

\subsection{Summary}

The approach and results presented in this thesis demonstrate a good foundation for control of the 12 actuated degree of freedom robot referred to as Micro Hydraulic Toolkit. Considering the physical robot was not accessible during the course of this thesis, a preliminary model was created to set a starting point for testing, based on MHT's kinematic information provided by DRDC. The preliminary model was created in MSC.ADAMS to prove the concept of the designed controller, shown in chapter 2. Following this, a professionally created simulation model in LMS software was made available for more rigorous and thorough controller testing.

The control method presented in chapter 3 uses MHT's kinematics to calculate the necessary joint rates to achieve the desired posture and trajectory inputs. The effectiveness of this control strategy is first tested by performing an aggressive manoeuvre attempted with MHT in the previous work at DRDC. The manoeuvre involves performing posture changes where the height of MHT alternates between $0.08 \mathrm{~m}$ to $0.632 \mathrm{~m}$ in eight seconds. This manoeuvre is achieved accurately with the proposed controller with very little error and no runaway issues, as was previously encountered. Additionally, an uneven terrain topology was created within the modelling software LMS to show MHT's terrain adaptation effectiveness. The terrain created is sagittally 
asymmetric with incline slopes as high as 24 degrees. With the prescribed gains, MHT successfully accommodates the terrain while minimizing roll and pitch angles of the platform. Additionally, the controller algorithm is equipped to allow independent leg control to allow MHT to perform a stepping motion to overcome obstacles that would otherwise deter wheeled locomotion. MHT effectively negotiated a $0.254 \mathrm{~m}$ high step while maintaining a statically stable stance through time-dependent posture and trajectory parameters. Though the outcome indicates that the controller worked effectively, it must be noted that the results originate from simulation model and not from the physical system.

In addition to the described control method, a basic optimization strategy is also applied to improve MHT's autonomy over uneven terrain. The optimization functions on-line without the use of exteroceptive sensors. The predominant goal of this process is to minimize the quasi-static torques occurring at the hip and knee actuators due to MHT's weight. The optimization determines the necessary posture parameters based on a prescribed objective function. This process is performed during regular time increments using the given kinematic state of MHT as an initial guess for the minimization process.

\subsection{Future Work}

This research has layed down a foundation for further controller development for MHT. However, there are a number of areas that should be considered when additional work is considered. 


\subsubsection{Simulation Improvements}

Because MHT is a very rigid robot, its sensory data is oscillatory, which directly affects the inputs used to control the platform. Future work might include incorporating a filter on the sensor data to smooth out the response of MHT. This is especially important for implementation on the physical system, considering the inaccuracies that occur in encoder readings for actuators or platform velocity and angular velocity values. A filter, both in simulation and on the real robot would limit the possibility of the actuators moving unexpectedly and/or erratically.

\subsubsection{Additional features on physical robot}

The presented controller uses two separate techniques for driving the wheels and stepping with the legs, however it is important that MHT is able to discern when to apply either method. Thus, another vital requirement for both the simulation and the physical system is to install exteroceptive sensors, to model them in simulation and to design a high-level control strategy based on the additional sensory information. This would provide MHT with the ability to better avoid obstacles and improve its autonomy.

\subsubsection{Model Verification}

Though the manoeuvres performed demonstrate consistent results and represent a good basis for trials on the actual robot, there is no doubt that there will be discrepancies with the real robot's outcome. For this reason, model verification must be conducted to assure the simulation model is as close a representation as possible of the real robot. This will 
aid in fine-tuning the simulation model to offer easier methods of controller design and testing.

\subsubsection{Controller Extension and Improvements}

This controller is largely influenced by the gains prescribed both in posture parameters and in the PD controller for calculating the voltage. Even though the gains selected offer good results, they have only been hand tuned. Thus, to further improve the performance of the robot, a method should be applied to effectively tune or to automatically choose the gains. In addition to this, the manoeuvres shown in this thesis were only tested on smooth terrain, and hence the assumption of constant contact forces. Therefore, it would be beneficial to examine simulation trials on different surface types, such as sand or snow. Other areas of interest, in terms of terrain, might be dealing with more challenging obstructions, such as higher steps and more complex obstacles. These challenges would certainly improve the effectiveness of the proposed controller. 


\section{References}

[1] J.L. Jones, "Robots at the tipping point - The road to the iRobot roomba." IEEE Robotics \& Automation Magazine, 2006. 13(1): p. 76-78.

[2] iRobot Corporation. Roomba [cited 2011 July]; www.irobot.com.

[3] SuperDroid Robots. Crawler [cited 2011 July]; www.superdroidrobots.com.

[4] A. Mutka and Z. Kovacic, "A Leg-Wheel Robot-Based Approach to the Solution of Flipper-Track Robot Kinematics", in IEEE Multi-coference on Systems and Control, 2011: Denver, Colorado, USA.

[5] R.J.Wood, "The first takeoff of a biologically inspired at-scale robotic insect." IEEE Transactions on Robotics, 2008. 24(2): p. 341-347.

[6] S.H. Hyon and T. Mita, "Development of a biologically inspired hopping robot "Kenken"." IEEE International Conference on Robotics and Automation, Vols IIv, Proceedings, 2002: p. 3984-3991.

[7] J.E. Clark, et al., "Biomimetic design and fabrication of a hexapedal running robot." IEEE International Conference on Robotics and Automation, Vols I-Iv, Proceedings, 2001: p. 3643-3649.

[8] U. Saranli, M. Buehler, and D.E. Koditschek, "RHex: A simple and highly mobile hexapod robot." International Journal of Robotics Research, 2001. 20(7): p. 616631.

[9] D. Campbell and M. Buehler, "Stair descent in the simple hexapod 'RHex'." IEEE International Conference on Robotics and Automation, Vols 1-3, Proceedings, 2003: p. 1380-1385.

[10] McGill University. RHex: Robotic Hexapod [cited 2010 October]; http://www.cim.mcgill.ca/ neville/RHex/indexRHex.html.

[11] H.Yoshida et al., "Mobile manipulation of humanoid robots - Optimal posture for generating large force based on statics." IEEE International Conference on Robotics and Automation, Vols I-Iv, Proceedings, 2002: p. 2271-2276.

[12] J.Chestnutt et al., "Footstep planning for the Honda ASIMO humanoid." IEEE International Conference on Robotics and Automation (ICRA), Vols 1-4, 2005: p. 629-634.

[13] Honda Motor Corporation. ASIMO website [cited 2011 July]; http://world.honda.com/ASIMO/. 
[14] Boston Dynamics. PETMAN [cited 2011 July]; http://www.bostondynamics.com/robot_petman.html.

[15] V.G. Loc et al., "Sensing and Control of Quadruped Walking and Climbing Robot Over Complex Environment." IEEE International Conference on Robots and Intelligent Systems, Vols 1-3, Conference Proceedings, 2008: p. 3884-3889.

[16] V.G. Loc et al., "Sensing and Gait Planning of Quadruped Walking and Climbing Robot for traversing in Complex Environment." Robotics and Autonomous Systems, 2010. 45: p. 666-675.

[17] A. Shkolnik and R. Tedrake, "Inverse kinematics for a point-foot quadruped robot with dynamic redundancy resolution." Proceedings of the IEEE International Conference on Robotics and Automation, Vols 1-10, 2007: p. 4331-4336.

[18] A. Shkolnik et al., "Bounding on rough terrain with the LittleDog robot." International Journal of Robotics Research, 2011.30(2): p. 192-215.

[19] Boston Dynamics LittleDog [cited 2011 July]; http://www.bostondynamics.com/robot_littledog.html.

[20] D. Wooden et al., "Autonomous Navigation for BigDog." IEEE International Conference on Robotics and Automation (ICRA), 2010: p. 4736-4741.

[21] Boston Dynamics. BigDog [cited 2011 July]; http://www.bostondynamics.com/robot_bigdog.html.

[22] J. Estremera and K.J. Waldron, "Thrust Control, Stabilization and Energetics of a Quadruped Running Robot." The International Journal of Robotics Research, 2008. 27(10): p. 1135-1151.

[23] R. Siegwart et al., "Innovative design for wheeled locomotion in rough terrain." Robotics and Autonomous Systems, 2002. 40(2-3): p. 151-162.

[24] J.J. Yuan and S. Hirose, "Research on leg-wheel hybrid stair-climbing robot, Zero Carrier." Proceedings of the IEEE International Conference on Robotics and Biomimetics, 2004: p. 654-659.

[25] BlueBotics. Shrimp [cited 2011 July]; http://www.bluebotics.com/.

[26] Hirose-Fukushima Robotics Lab. Zero-Carrier [cited 2011 July]; http://wwwrobot.mes.titech.ac.jp/robot/walking/zerocarrier/zerocarrier_e.html.

[27] J.A. Smith et al., "Bounding with Active Wheels and Liftoff Angle Velocity Adjustment.", International Journal of Robotics Research, 2010. 29(4): p. 414427. 
[28] M. Faragalli, I. Sharf, and M. Trentini, "Velocity Control of a Hybrid Quadruped Bounding Robot." IEEE International Conference on Robots and Intelligent Systems, Vols 1-3, Conference Proceedings, 2008: p. 1501-1506.

[29] S.J.Ylonen and A. Halme, "Further development and testing of the hybrid locomotion of WorkPartner robot." Climbing and Walking Robots, 2002: p. 293298.

[30] A. Halme, I. Leppanen, and S. Salmi, "Development of WorkPartner-robot design of actuating and motion control system." Proceedings of the $2 n d$ International Conference on Climbing and Walking Robots, CLAWAR 99, 1999: p. 657-665.

[31] A. Halme, T. Luksch, and S. Ylonen, "Biomimicing motion control of the WorkPartner robot." Industrial Robot-an International Journal, 2004. 31(2): p. 209-217.

[32] G. Besseron et al., "Decoupled control of the high mobility robot Hylos based on a dynamic stability margin." IEEE International Conference on Robots and Intelligent Systems, Vols 1-3, Conference Proceedings, 2008: p. 2435-2440.

[33] G. Besseron et al., "Stability control of an hybrid wheel-legged robot." Climbing and Walking Robots, 2006: p. 533-540.

[34] C. Grand, F. Ben Amara, and P. Bidaud, "Kinematic analysis and stability optimisation of a reconfigurable legged-wheeled mini-rover." Unmanned Ground Vehicle Technology Iv, 2002. 4715: p. 295-302.

[35] C. Grand, F. Benamar, and F. Plumet, "Motion kinematics analysis of wheeledlegged rover over 3D surface with posture adaptation." Mechanism and Machine Theory, 2010. 45(3): p. 477-495.

[36] C. Grand et al., "Decoupled control of posture and trajectory of the hybrid wheellegged robot Hylos." IEEE International Conference on Robotics and Automation, Vols 1- 5, Proceedings, 2004: p. 5111-5116.

[37] C. Grand et al., "Stability and traction optimization of a reconfigurable wheellegged robot." International Journal of Robotics Research, 2004. 23(10-11): p. 1041-1058.

[38] R.K. Barai and K. Nonami, "Locomotion control of a hydraulically actuated hexapod robot by robust adaptive fuzzy control with self-tuned adaptation gain and dead zone fuzzy pre-compensation." Journal of Intelligent \& Robotic Systems, 2008. 53(1): p. 35-56. 
[39] R.K. Barai and K. Nonami, "Optimal two-degree-of-freedom fuzzy control for locomotion control of a hydraulically actuated hexapod robot." Information Sciences, 2007. 177(8): p. 1892-1915.

[40] T. Doi et al., "Development of a quadruped walking robot to work on steep slopes, TITAN XI (Walking motion with compensation for compliance)." IEEE International Conference on Intelligent Robots and Systems, Vols 1-4, 2005: p. 3413-3418.

[41] Hirose-Fukushima Robotics Lab. TITAN-XI [cited 2011 July]; http://wwwrobot.mes.titech.ac.jp/robot/walking/titan11/titan11_e.html.

[42] B. Beckman, "Predicted Dynamic Stability of the Varying Center of Gravity Micro Hydraulic Toolkit Platform", in Department of Mechanical and Manufacturing Engineering, 2008, University of Calgary: Calgary.

[43] B. Beckman and M. Trentini, "Kinematic Range of Motion Analysis for a High Degree-of-Freedom Unmanned Ground Vehicle", 2009, Defence Research and Development Canada - Suffield: Medicine Hat.

[44] B. Beckman, M. Trentini, and J. Pieper. "Control algorithms for stable range-ofmotion behaviours of a multi degree-of-freedom robot." International Conference on Autonomous and Intelligent Systems (AIS). 2010.

[45] H. Li, "A software framework for control of UGVS in complex terrain." IASTED International Conference on Robotics and Applications. 2009.

[46] P. Donelan, "Kinematic Singularities of Robot Manipulators", in Advances in Robot Manipulators, E. Hall, Editor, 2010, InTech.

[47] K. Iagnemma et al., "Mobile robot kinematic reconfigurability for rough-terrain." Sensor Fusion and Decentralized Control in Robotic Systems III, 2000. 4196: p. 413-420.

[48] J. Angeles, "Rational Kinematics. Vol. 34. 1988: Springer-Verlag. 137.

[49] M. Nahon, "Optimization of Force Destribution in Redundantly-Actuated Robotic Systems", in Department of Mechanical Engineering, 1991, McGill University: Montreal. p. 225.

[50] J. Smith, "Galloping, Bounding and Wheeled-Leg Modes of Locomotion on Underactuated Quadrupedal Robots", 2008, McGill University: Montreal.

[51] A.D. Belegundu and T.R. Chandrupatla, "Optimization Concepts and Applications in Engineering", 1999, Upper Saddle River, N.J.: Prentice Hall. 
Appendix A

MSC.ADAMS model details

\begin{tabular}{|c|c|c|c|}
\hline Part & Mass (kg) & Approx. Dimensions (m) & Inertias $\left(\mathrm{kg} \cdot \mathrm{m}^{2}\right)^{*}$ \\
\hline Platform & 100 & $\begin{array}{c}\mathrm{L} \times \mathrm{W} \times \mathrm{H} \\
0.7 \times 0.5 \times 0.23\end{array}$ & $\begin{array}{l}\mathrm{I}_{\mathrm{xx}}=4.755 \\
\mathrm{I}_{\mathrm{yy}}=2.653 \\
\mathrm{I}_{z z}=6.481\end{array}$ \\
\hline Femur & 1 & $\begin{array}{c}\mathrm{L} \times \mathrm{W} \times \mathrm{H} \\
0.315 \times 0.105 \times 0.054\end{array}$ & $\begin{array}{l}\mathrm{I}_{\mathrm{xx}}=9.188 \times 10^{-3} \\
\mathrm{I}_{\mathrm{yy}}=8.512 \times 10^{-2} \\
\mathrm{I}_{\mathrm{zz}}=1.162 \times 10^{-3}\end{array}$ \\
\hline Tibia & 6 & $\begin{array}{c}\mathrm{L} \times \mathrm{W} \times \mathrm{H} \\
0.377 \times 0.105 \times 0.054\end{array}$ & $\begin{array}{c}\mathrm{I}_{\mathrm{xx}}=0.077 \\
\mathrm{I}_{\mathrm{yy}}=0.0725 \\
\mathrm{I}_{\mathrm{zz}}=6.970 \times 10^{-3}\end{array}$ \\
\hline Wheel & 4.2 & $\begin{array}{l}\text { Radius: } 0.127 \\
\text { Width: } 0.1016\end{array}$ & $\begin{array}{l}\mathrm{I}_{\mathrm{xx}}=2.055 \times 10^{-2} \\
\mathrm{I}_{\mathrm{yy}}=3.387 \times 10^{-2} \\
\mathrm{I}_{\mathrm{zz}}=2.055 \times 10^{-2}\end{array}$ \\
\hline
\end{tabular}

* For the platform, femur and tibia, the $z$-axis is aligned along the length of the part for determining the inertias. For the wheel, the $y$-axis lies along the wheel's axis of rotation. 